\title{
On the smooth transfer for Guo-Jacquet relative trace formulae
}

\author{
Chong Zhang
}

\begin{abstract}
We establish the existence of smooth transfer for Guo-Jacquet relative trace formulae in $p$-adic case. This kind of smooth transfer is a key step towards a generalization of Waldspurger's result on central values of Lfunctions of $\mathrm{GL}_{2}$.
\end{abstract}

\section{Introduction}

History The periods of automorphic forms play an important role in the study of automorphic representations and related number theoretic problems. For example, people believe that periods of automoprhic forms can characterize the Langlands functoriality of automorphic representations. Recently, Y. Sakellaridis and A. Venkatesh [SV] developed an ambitious program, the so-called relative Langlands program, on this aspect. There are several powerful tools to study periods. The theory of relative trace formula is one of them, which was first studied by H. Jacquet. In [Ja1], Jacquet reproved a remarkable result of J.-L. Waldspurger [Wa1] on central values of L-functions of $\mathrm{GL}_{2}$ by comparing relative trace formulae on different groups. In [Gu1], J. Guo and Jacquet made a conjecture (see Conjecture 1.1) generalizing Waldspurger's result to higher rank cases.

To be precise, let $k$ be a number field, $\mathbb{A}$ its ring of adeles. Consider $\mathbf{G}=$ $\mathrm{GL}_{2 n}$ and $\mathbf{H}=\mathrm{GL}_{n} \times \mathrm{GL}_{n}$ embedded into $\mathbf{G}$ diagonally, which are reductive groups over $k$. Let $k^{\prime}$ be a quadratic field extension of $k, \eta$ the quadratic character of $\mathbb{A}^{\times} / k^{\times}$attached to $k^{\prime}$ by class field theory. Let $\mathbf{Z}$ be the center of $\mathbf{G}$. When we say a cuspidal representation $\pi$, we always mean that $\pi$ is irreducible and automorphic. For a cuspidal representation $\pi$ of $\mathbf{G}(\mathbb{A})$, we consider the linear forms $\ell_{\mathbf{H}}$ and $\ell_{\mathbf{H}, \eta}$ on $\pi$ defined by periods:

$$
\ell_{\mathbf{H}}(\phi):=\int_{\mathbf{H}(k) \mathbf{Z}(\mathbb{A}) \backslash \mathbf{H}(\mathbb{A})} \phi(h) \mathrm{d} h, \quad \ell_{\mathbf{H}, \eta}(\phi):=\int_{\mathbf{H}(k) \mathbf{Z}(\mathbb{A}) \backslash \mathbf{H}(\mathbb{A})} \phi(h) \eta(h) \mathrm{d} h,
$$

where $\phi \in \pi$ and $\eta(h):=\eta(\operatorname{det} h)$. We say that $\pi$ is $\mathbf{H}$-distinguished (resp. $(H, \eta)$-distinguished) if $\ell_{\mathbf{H}} \neq 0$ (resp. $\ell_{\mathbf{H}, \eta} \neq 0$ ). On the other hand, for a quaternion algebra $\mathrm{D}$ over $k$ containing $k^{\prime}$, let $\mathbf{G}^{\prime}=\mathbf{G}_{\mathrm{D}}^{\prime}=\mathrm{GL}_{n}(\mathrm{D})$ and $\mathbf{H}^{\prime}=\mathrm{GL}_{n}\left(k^{\prime}\right)$, both viewed as reductive groups defined over $k$. View $\mathbf{H}^{\prime}$ as a subgroup of $\mathbf{G}^{\prime}$ in the natural way and identify the center of $\mathbf{G}^{\prime}$ with $\mathbf{Z}$. For a cuspidal representation $\pi^{\prime}$ of $\mathbf{G}^{\prime}(\mathbb{A})$, consider the linear form $\ell_{\mathbf{H}^{\prime}}$ on $\pi^{\prime}$ defined by

$$
\ell_{\mathbf{H}^{\prime}}(\phi):=\int_{\mathbf{H}^{\prime}(k) \mathbf{Z}(\mathbb{A}) \backslash \mathbf{H}^{\prime}(\mathbb{A})} \phi(h) \mathrm{d} h, \quad \phi \in \pi^{\prime} .
$$


We say that $\pi^{\prime}$ is $\mathbf{H}^{\prime}$-distinguished if $\boldsymbol{\ell}_{\mathbf{H}^{\prime}} \neq 0$.

Denote by $X\left(k^{\prime}, k\right)$ the set of quaternion algebras D over $k$ containing $k^{\prime}$. For a cuspidal representation $\pi$ of $\mathbf{G}(\mathbb{A})$, denote by $X\left(k^{\prime}, k ; \pi\right)$ the subset of $X\left(k^{\prime}, k\right)$ such that the Jacquet-Langlands correspondence $\pi_{\mathrm{D}}^{\prime}:=\mathrm{JL}(\pi)$ of $\pi$ exists as a cuspidal representation of $\mathbf{G}_{\mathrm{D}}^{\prime}(\mathbb{A})$.

Motivated by Waldspurger's result in the case $n=1$, the following conjecture was made in [Gul].

Conjecture 1.1 ((Guo-Jacquet)). Fix a cuspidal representation $\pi$ of $\mathbf{G}(\mathbb{A})$.

1. Fix a quaternion algebra D in $X\left(k^{\prime}, k ; \pi\right)$. Suppose that $\pi_{\mathrm{D}}^{\prime}$ is $\mathbf{H}^{\prime}$-distinguished. Then $\pi$ is both $\mathbf{H}$-distinguished and $(\mathbf{H}, \eta)$-distinguished.

2. Suppose that $n$ is odd and $\pi$ is both $\mathbf{H}$-distinguished and $(\mathbf{H}, \eta)$-distinguished. Then there exists $\mathrm{D} \in X\left(k^{\prime}, k ; \pi\right)$ such that $\pi_{\mathrm{D}}^{\prime}$ is $\mathbf{H}^{\prime}$-distinguished.

Moreover, when $n$ is even, with more restriction, the direction (ii) of Conjecture 1.1 should also hold. We refer the reader to [FM, Conjecture 3] and [FMW, Conjecture 1.5] for more information.

The periods defined above can be used to study the central value $L\left(\frac{1}{2}, \pi_{k^{\prime}}\right)=$ $L\left(\frac{1}{2}, \pi\right) L\left(\frac{1}{2}, \pi \otimes \eta\right)$ where $\pi_{k^{\prime}}$ is the base change of $\pi$ to $\mathbf{G}\left(\mathbb{A}_{k^{\prime}}\right)$. It was shown in [FJ] that if $\pi$ is both $\mathbf{H}$-distinguished and $(\mathbf{H}, \eta)$-distinguished then $L\left(\frac{1}{2}, \pi_{k^{\prime}}\right) \neq$ 0 . One also expects that there exists a relation between this L-value and the period $\ell_{\mathbf{H}^{\prime}}$ on $\pi^{\prime}$.

In [Gu1], a relative trace formula approach called Guo-Jacquet relative trace formulae today, which is a natural extension of Jacquet's method in [Ja1], was proposed to attack the above conjecture. The first step, that is, the fundamental lemma for unit Hecke functions, has also been established by [Gu1]. The smooth transfer can be viewed as the second step on the geometric side of Guo-Jacquet relative trace formulae. Since we only focus on the smooth transfer, which is a local issue, we will not recall the precise form of Guo-Jacquet relative trace formulae, which is a global issue. We refer the reader to [Gu1] or [FMW] for more details.

Very recently, B. Feigon, K. Martin and D. Whitehouse [FMW] obtained some partial results on Conjecture 1.1, by using a simple form of Guo-Jacquet trace formulae. They showed the existence of smooth transfer for BruhatSchwartz functions satisfying certain specific properties. Of course, one has to show the existence of smooth transfer for the full space of Bruhat-Schwartz functions, if one aims to prove Conjecture 1.1 completely. Due to our result, one can remove some conditions of the results in [FMW], as [FMW, Remark $6.2]$ states.

There is also a generalization of Waldspurger's result in another direction: the so called Gan-Gross-Prasad conjecture [GGP] and the refined version of it by Ichino-Ikeda [II] in the case of orthogonal groups and by N. Harris [Ha] in the case of unitary groups. Recently, W. Zhang ([Zhw1],[Zhw2]) has made a great advance towards the global Gan-Gross-Prasad conjecture for unitary groups by using the relative trace formula developed by Jacquet and S. Rallis. One of his achievements is his proof of the smooth transfer conjecture in $p$-adic case for the Jacquet-Rallis relative trace formula. His method is close to that of [Ja2]. The several remarkable successes on the Gan-Gross-Prasad conjecture, both in local and global directions, will shed some light on the problem considered here. 
Results of this article In this article, we establish the existence of smooth transfer in $p$-adic case for Guo-Jacquet relative trace formulae. Let us briefly explain what the smooth transfer means. From now on, let $F$ be a $p$-adic field, which is a completion of $k$ at a finite place. Let $E$ be a quadratic field extension of $F$ and $\mathrm{D}$ a quaternion algebra over $F$ containing $E$. Notice that such quaternion algebras are parameterized by $F^{\times} / \mathrm{NE} E^{\times}$, where $\mathrm{N}$ is the norm map from $E^{\times}$to $F^{\times}$. When we want to emphasize the dependence of $\mathrm{D}$ on $\epsilon \in F^{\times} / \mathrm{N} E^{\times}$, we write $\mathrm{D}_{\epsilon}$. Let $\eta$ be the quadratic character of $F^{\times}$associated to $E / F$. We define $(\mathbf{G}, \mathbf{H})$ and $\left(\mathbf{G}^{\prime}, \mathbf{H}^{\prime}\right)$ over $F$ in the same way as the global situation. Write $G=\mathbf{G}(F), H=\mathbf{H}(F), G^{\prime}=\mathbf{G}^{\prime}(F)$ and $H^{\prime}=\mathbf{H}^{\prime}(F)$.

The group $H \times H$ (resp. $H^{\prime} \times H^{\prime}$ ) acts on $G$ (resp. $G^{\prime}$ ) by left and right translations. With respect to this action, we can talk about the notion of $H \times H$ or $H^{\prime} \times H^{\prime}$-regular semisimple (cf. $\S 3.1$ ) elements in $G$ or $G^{\prime}$ respectively. Denote by $G_{\mathrm{rs}}$ and $G_{\mathrm{rs}}^{\prime}$ the set of the regular and semisimple elements in $G$ and $G^{\prime}$ respectively. Then there is a natural injection (cf. Proposition 5.1)

$$
\left[G_{\mathrm{rs}}^{\prime}\right] \hookrightarrow\left[G_{\mathrm{rs}}\right]
$$

from the set of $H^{\prime} \times H^{\prime}$-orbits in $G_{\mathrm{rs}}^{\prime}$ to the set of $H \times H$-orbits in $G_{\mathrm{rs}}$. We say that $x \in G_{\mathrm{rs}}$ matches $y \in G_{\mathrm{rs}}^{\prime}$ and write $x \leftrightarrow y$ if the orbit of $y$ goes to that of $x$ under this injection. We say that $x \in G_{\mathrm{rs}}$ comes from $G_{\mathrm{rs}}^{\prime}$ if there exists $y \in G_{\mathrm{rs}}^{\prime}$ such that $x \leftrightarrow y$. If $x \leftrightarrow y$, their stabilizers denoted by $(H \times H)_{x}$ and $\left(H^{\prime} \times H^{\prime}\right)_{y}$ are isomorphic. Fix a Haar measure on $H$ and a Haar measure on $(H \times H)_{x}$ for each $x \in G_{\mathrm{rs}}$. Note that $\left.\eta\right|_{(H \times H)_{x}}=1$. For each $f \in \mathcal{C}_{c}^{\infty}(G)$, define the orbital integral of $f$ at $x$ to be

$$
O^{\eta}(x, f)=\int_{(H \times H)_{x} \backslash H \times H} f\left(h_{1}^{-1} x h_{2}\right) \eta\left(\operatorname{det} h_{2}\right) \mathrm{d} h_{1} \mathrm{~d} h_{2} .
$$

We can define a transfer factor $\kappa$ (cf. Defintion 5.7) which is a function on $G_{\mathrm{rs}}$ so that $\kappa(\cdot) O^{\eta}(\cdot, f)$ only depends on the $H \times H$-orbits in $G_{\mathrm{rs}}$. Similarly, fix a Haar measure on $H^{\prime}$. We fix the Haar measure on $\left(H^{\prime} \times H^{\prime}\right)_{y}$ for each $y \in G_{\mathrm{rs}}^{\prime}$ so that it is compatible with that on $(H \times H)_{x}$ if $x \leftrightarrow y$. For each $f^{\prime} \in \mathcal{C}_{c}^{\infty}\left(G^{\prime}\right)$, define the orbital integral of $f^{\prime}$ at $y$ to be

$$
O\left(y, f^{\prime}\right)=\int_{\left(H^{\prime} \times H^{\prime}\right)_{y} \backslash H^{\prime} \times H^{\prime}} f^{\prime}\left(h_{1}^{-1} y h_{2}\right) \mathrm{d} h_{1} \mathrm{~d} h_{2} .
$$

For $f \in \mathcal{C}_{c}^{\infty}(G)$ and $f^{\prime} \in \mathcal{C}_{c}^{\infty}\left(G^{\prime}\right)$, we say that $f$ and $f^{\prime}$ are smooth transfer of each other if

$$
\kappa(x) O^{\eta}(x, f)=\left\{\begin{aligned}
O\left(y, f^{\prime}\right), & \text { if there exists } y \in G_{\mathrm{rs}}^{\prime} \text { such that } x \leftrightarrow y \\
0, & \text { otherwise. }
\end{aligned}\right.
$$

Denote by $\mathcal{C}_{c}^{\infty}(G)_{0}$ the subspace of elements $f$ in $\mathcal{C}_{c}^{\infty}(G)$ satisfying that $O^{\eta}(x, f)=$ 0 for any $x \in G_{\mathrm{rs}}$ that does not come from $G_{\mathrm{rs}}^{\prime}$.

Our main result is the following theorem.

Theorem 1.2. For each $f^{\prime} \in \mathcal{C}_{c}^{\infty}\left(G^{\prime}\right)$, there exists $f \in \mathcal{C}_{c}^{\infty}(G)$ that is a smooth transfer of $f^{\prime}$. Conversely, for each $f \in \mathcal{C}_{c}^{\infty}(G)_{0}$, there exists $f^{\prime} \in \mathcal{C}_{c}^{\infty}\left(G^{\prime}\right)$ that is a smooth transfer of $f$. 
Now we explain how to reduce the existence of smooth transfer for functions on groups to the existence of smooth transfer for functions on symmetric spaces. This reduction is a standard procedure.

There is an involution $\theta$ on $\mathbf{G}$ such that $\mathbf{H}=\mathbf{G}^{\theta}$ is the subgroup of $\mathbf{G}$ fixed by $\theta$. Let $S:=G / H$ be the $p$-adic symmetric space associated to $(\mathbf{G}, \mathbf{H})$. The group $H$ acts on $S$ by the conjugate action. There is a symmetrization map $s: \mathbf{G} \rightarrow \mathbf{G}^{\iota}$, where $\iota$ is the anti-involution on $\mathbf{G}$ defined by $\iota(g)=\theta\left(g^{-1}\right)$ and $\mathbf{G}^{\iota}$ is the subgroup fixed by $\iota$. The symmetrization map is given by $s(g)=g \iota(g)$. Via the map $s$, we view $S$ as a subset of $\mathbf{G}^{\iota}(F)$. An element $g \in G$ is $H \times H$ regular semisimple if and only if $x=s(g) \in S$ is $H$-regular semisimple. Denote by $S_{\text {rs }}$ the subset of regular semisimple elements in $S$. Let $q: \mathcal{C}_{c}^{\infty}(G) \rightarrow \mathcal{C}_{c}^{\infty}(S)$ be the natural surjection map defined by

$$
(q f)(x)=\int_{H} f(g h) \mathrm{d} h
$$

if $x=s(g)$. Let $x=s(g) \in S$ be regular semisimple. Then its stabilizer $H_{x}$ is isomorphic to $(H \times H)_{g}$. We choose the same Haar measure on $H$ as before and the Haar measure on $H_{x}$ compatible with that on $(H \times H)_{g}$. For $\tilde{f} \in \mathcal{C}_{c}^{\infty}(S)$, define the orbital integral of $\tilde{f}$ at $x$ to be

$$
O^{\eta}(x, \tilde{f})=\int_{H_{x} \backslash H} \tilde{f}\left(h^{-1} x h\right) \eta(\operatorname{det} h) \mathrm{d} h .
$$

We define a transfer factor on $S_{\text {rs }}$ so that $\kappa(x)=\kappa(g)$ if $x=s(g)$. Then, by a routine computation, we have

$$
\kappa(g) O^{\eta}(g, f)=\kappa(x) O^{\eta}(x, \tilde{f})
$$

for each $f \in \mathcal{C}_{c}^{\infty}(G), \tilde{f}=q f \in \mathcal{C}_{c}^{\infty}(S)$ and $x=s(g) \in S_{\text {rs }}$. Thus, the study of orbital integrals for $\mathcal{C}_{c}^{\infty}(G)$ with respect to $H \times H$-action is equivalent to that of orbital integrals for $\mathcal{C}_{c}^{\infty}(S)$ with respect to $H$-action. Similarly, the study of orbital integrals for $\mathcal{C}_{c}^{\infty}\left(G^{\prime}\right)$ with respect to $H^{\prime} \times H^{\prime}$-action is equivalent to that of orbital integrals for $\mathcal{C}_{c}^{\infty}\left(S^{\prime}\right)$ with respect to $H^{\prime}$-action, where $S^{\prime}:=G^{\prime} / H^{\prime}$ is the $p$-adic symmetric space associated to $\left(\mathbf{G}^{\prime}, \mathbf{H}^{\prime}\right)$.

There is a natural injection (cf. Proposition 5.1)

$$
\left[S_{\mathrm{rs}}^{\prime}\right] \hookrightarrow\left[S_{\mathrm{rs}}\right]
$$

from the set of $H^{\prime}$-orbits in $S_{\mathrm{rs}}^{\prime}$ to the set of $H$-orbits in $S_{\mathrm{rs}}$. We say that $x \in S_{\mathrm{rs}}$ matches $y \in S_{\mathrm{rs}}^{\prime}$ and write $x \leftrightarrow y$ if the orbit of $y$ goes to that of $x$. Similarly, for $f \in \mathcal{C}_{c}^{\infty}(S)$ and $f^{\prime} \in \mathcal{C}_{c}^{\infty}\left(S^{\prime}\right)$, we can define the notion of smooth transfer for them (see $\S 5.1$ for more details). Then we immediately see that Theorem 1.2 is equivalent to Theorem 5.13 which claims the existence of smooth transfer at the level of symmetric spaces.

There is also the notion of smooth transfer at the level of Lie algebras, called the Lie algebra version of smooth transfer. Here, for Lie algebras, we mean the tangent spaces $\mathfrak{s}$ and $\mathfrak{s}^{\prime}$ of $\mathbf{G} / \mathbf{H}$ and $\mathbf{G}^{\prime} / \mathbf{H}^{\prime}$ at the identity respectively. The notion of smooth transfer in this version is determined by the orbital integrals with respect to adjoint actions of $H$ and $H^{\prime}$ on $\mathfrak{s}(F)$ and $\mathfrak{s}^{\prime}(F)$ respectively. We refer the reader to $\S 5$ for more details. 
Our method to showing the existence of smooth transfer is mainly inspired by Zhang's work [Zhw1] on the smooth transfer for the Jacquet-Rallis relative trace formula and Waldspurger's work [Wa3] on the endoscopic smooth transfer for Arthur's stable trace formula. First, we reduce Theorem 5.13 to Theorem 5.14 which claims the existence of smooth transfer at the level of Lie algebras. Next we reduce Theorem 5.14 to Theorem 5.16 which asserts that the Fourier transform preserves smooth transfer up to a nonzero scalar. The several reduction steps here almostly follow those of [Zhw1]. To prove Theorem 5.16, since the absence of a suitable partial Fourier transform, we could not adapt the inductive argument in [Zhw1, §4.4] any more. Our approach is more close to that of [Wa3] where a global argument emerged. However there are still some differences between our method and that of [Wa3]. These differences are caused by the following facts. The first fact is that

$$
\left[\mathfrak{s}_{\mathrm{rs}}(F)\right] \supseteqq \bigcup_{\epsilon \in F^{\times} / \mathrm{N} E^{\times}}\left[\mathfrak{s}_{\epsilon, \mathrm{rs}}^{\prime}(F)\right]
$$

where $\mathfrak{s}_{\epsilon}^{\prime}$ is the Lie algebra associated to $\left(\mathbf{G}_{\epsilon}^{\prime}=\mathrm{GL}_{n}\left(\mathrm{D}_{\epsilon}\right), \mathbf{H}^{\prime}\right)$ and $\left[\mathfrak{s}_{\epsilon, \mathrm{rs}}^{\prime}(F)\right]$ (resp. $\left[\mathfrak{s}_{\mathrm{rs}}(F)\right]$ ) is the set of $H^{\prime}$ - (resp. $H$ )-regular semisimple orbits. The above two sets are equal if and only if $n=1$. Even worse, the elliptic parts of the above two sets are equal if and only $n$ is odd. These phenomenons are unlike other cases of relative trace formulae. Now suppose that we are in the global setting. The second fact is that if $X_{0}$ is a global element in $\mathfrak{s}_{\mathrm{rs}}(k)$ which does not come from $\mathfrak{s}_{\mathrm{rs}}^{\prime}(k)$ then there exist at least two places $v_{1}, v_{2}$ such that $X_{0}$ does not come from $\mathfrak{s}_{\mathrm{rs}}^{\prime}\left(k_{v_{1}}\right)$ or $\mathfrak{s}_{\mathrm{rs}}^{\prime}\left(k_{v_{2}}\right)$. This is unlike the case of endoscopic transfer and prevents us to use global method to prove Theorem 8.1 which asserts that the orbital integral $O^{\eta}(X, \widehat{f})=0$ for $X \in \mathfrak{s}_{\mathrm{rs}}(F)$ not coming from $\mathfrak{s}_{\mathrm{rs}}^{\prime}(F)$ where $f \in \mathcal{C}_{c}^{\infty}(\mathfrak{s}(F))$ is a smooth transfer of some element in $\mathcal{C}_{c}^{\infty}\left(\mathfrak{s}^{\prime}(F)\right)$ and $\widehat{f}$ is its Fourier transform. Instead we will use a pure local argument, which is due to the referee, to show Theorem 8.1.

To prove Theorem 5.16, we have to show the representability of the Fourier transform of orbital integrals as distributions (see Theorem 6.1), exhibit "limit formulae" for the kernel functions (see Proposition 7.1) as Waldspurger did in [Wa2], and also prove analogues of some results (see Proposition 7.6 and Theorem 8.4) in [Wa3]. These results, which are on harmonic analysis on certain $p$ adic symmetric spaces, maybe appear in the literature for the first time. We expect that the techniques developed in this paper should be probably generalized to treat some other similar open questions concerning relative trace formulae for symmetric pairs. Actually, we do successfully generalize this method to prove the existence of smooth transfer for other relative trace formula in [Zhc]. Here we mention some cases of symmetric pairs where our results in $\S 6$ and $\S 7$ should hold. Still let $E$ be a quadratic field extension of a $p$-adic field $F$. The first class of symmetric pairs are "inner forms" of $(\mathbf{G}, \mathbf{H})$ or $\left(\mathbf{G}^{\prime}, \mathbf{H}^{\prime}\right)$. Now let D be a central division algebra over $F$. Let $\mathbf{G}=\mathrm{GL}_{2 m}(\mathrm{D})$ and $\mathbf{H}=\mathrm{GL}_{m}(\mathrm{D}) \times \mathrm{GL}_{m}(\mathrm{D})$. Then $(\mathbf{G}, \mathbf{H})$ is the symmetric pair considered in [Zhc]. We can also consider the symmetric pair $(\mathbf{G}, \mathbf{H})=\left(\mathrm{GL}_{2 m}(\mathrm{D}), \mathrm{GL}_{m}\left(\mathrm{D} \otimes_{F} E\right)\right)$, or, more generally, the symmetric pair $(\mathbf{G}, \mathbf{H})=\left(\mathrm{GL}_{m}(\mathrm{D}), \mathrm{GL}_{m}\left(\mathrm{D}^{\prime}\right)\right)$ where $\mathrm{D}$ is a central simple algebra over $F$ containing $E$ and $\mathrm{D}^{\prime}$ is the centralizer of $E$ in $\mathrm{D}$. The second class of symmetric pairs are Galois symmetric pairs. Now let $\mathbf{H}$ be a connected reductive group over $F$, and $\mathbf{G}=\operatorname{Res}_{E / F}\left(\mathbf{H}_{E}\right)$ the Weil restriction of the base 
change of $\mathbf{H}$ to $E$. Then $(\mathbf{G}, \mathbf{H})$ is called a Galois symmetric pair.

Structure of this article In $\S 2$, we introduce some notations and conventions that are frequently used in the paper.

In $\S 3$, since $(\mathbf{G}, \mathbf{H})$ and $\left(\mathbf{G}^{\prime}, \mathbf{H}^{\prime}\right)$ are symmetric pairs, we collect some basic notions and results on symmetric pairs. In particular, we recall the analytic Luna Slice Theorem which plays an pivotal role on the reduction steps of the smooth transfer.

In $\S 4$, we study our specific symmetric pairs $(\mathbf{G}, \mathbf{H})$ and $\left(\mathbf{G}^{\prime}, \mathbf{H}^{\prime}\right)$ more concretely. We give a complete description of all the descendants of the corresponding symmetric spaces and their Lie algebras. We also prove Propositions 4.4 and 4.8 , which are about two inequalities. These inequalities are crucial for bounding the orbital integrals later (see Theorem 6.11).

In $\S 5$, we introduce the main issue of this article, that is, the smooth transfer at the level of symmetric spaces and its Lie algebra version. We explain why Theorem 5.16 implies Theorem 5.13. We also prove the fundamental lemma in the Lie algebra version, which is crucial for our global approach to prove Theorem 5.16.

In $\S 6$, to prove Theorem 5.16, we pay more effort on studying the Fourier transform of orbital integrals. One of the most important question is to show the representability, that is, the Fourier transform of an orbital integral considered as a distribution can be represented by a locally integrable kernel function. We deal with this issue in this section. The representability itself is also a fundamental question in harmonic analysis on $p$-adic symmetric spaces.

$\S 7$ is devoted to showing limit formulae for the kernel functions of the Fourier transform, which is an analogue of [Wa2, Section VIII]. We also construct certain good test functions which are smooth transfer of each other and whose Fourier transforms are also smooth transfer of each other up to a scalar. This construction is an analogue of [Wa3, Proposition 8.2]. Such test functions are used in the later construction of certain global Schwartz functions.

Finally, in $\S 8$, we finish the proof of Theorem 5.16, basing on the results of $\S 7$ and the fundamental lemma.

\section{Notations and conventions}

We now introduce some notations and conventions, which are frequently used in $\S 3-\S 7$.

Fields Let $F$ be a non-archimedean local field of characteristic 0 , with finite residue field. Fix an algebraic closure $\bar{F}$, and denote by $\Gamma_{F}=\operatorname{Gal}(\bar{F} / F)$ the absolute Galois group. We denote by $|\cdot|_{F}$ (resp. $v_{F}$ ) the absolute value (resp. the valuation) of $F$, and extend them to $\bar{F}$ in the usual way. Let $\mathcal{O}_{F}$ be the integer ring of $F$ and fix a uniformizer $\varpi$ of $\mathcal{O}_{F}$. For a finite extension field $L$ of $F$, denote by $\mathrm{N}_{L / F}$ and $\operatorname{Tr}_{L / F}$ the norm and trace maps respectively. Throughout this article, we fix a nontrivial additive unitary character $\psi: F \rightarrow \mathbb{C}^{\times}$.

Varieties and groups All the algebraic varieties and algebraic groups that we consider are defined over $F$ except in $\S 8$. We always use bold letter to denote an algebraic group, italic letter to denote its $F$-rational points, and Fraktur letter 
to denote its Lie algebra. For example, let $\mathbf{G}$ be a reductive group. We write $G=\mathbf{G}(F)$ and denote by $\mathfrak{g}$ the Lie algebra of $\mathbf{G}$. By a subgroup of $\mathbf{G}$, we mean a closed $F$-subgroup. We write $N_{G}(\cdot)$ for the normalizer and $Z_{G}(\cdot)$ for the centralizer of a certain set in $G$, and write $\mathbf{Z}$ for the center of $\mathbf{G}$. For an algebraic variety $\mathbf{X}, X=\mathbf{X}(F)$ is equipped with the natural topology induced from $F$. Thus, $X$ is a locally compact totally disconnected topological space. Sometimes we treat finite dimensional vector spaces defined over $F$ as algebraic varieties over $F$.

Heights Let $\mathbf{G}$ be a reductive group and $G=\mathbf{G}(F)$. Following HarishChandra, we define a height function $\|\cdot\|$ on $G$ valued in $\mathbb{R}_{\geq 1}$. If $\mathbf{T}$ is a sub-torus of $\mathbf{G}$ and $T=\mathbf{T}(F)$, denote by $\|\cdot\|_{T \backslash G}$ the induced height function on $G$. The precise definitions and some important properties of height functions are well discussed in [Ko, §18].

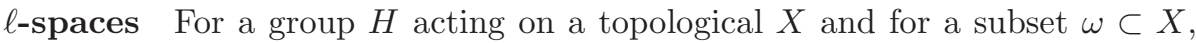
we denote by $\omega^{H}$ the set $\{h \cdot x: x \in \omega, h \in H\}$, and by $\operatorname{cl}(\omega)$ the closure of $\omega$ in $X$. For an element $x \in X$, we denote by $H_{x}$ the stabilizer of $x$ in $H$.

For a locally compact totally disconnected topological space $X$, we denote by $\mathcal{C}_{c}^{\infty}(X)$ the space of locally constant and compactly supported $\mathbb{C}$-valued functions, and by $\mathcal{D}(X)$ the space of distributions on $X$. For $f \in \mathcal{C}_{c}^{\infty}(X)$, we denote by $\operatorname{Supp}(f)$ its support. Suppose that $H$ (an $\ell$-group) acts on $X$. Then $H$ acts on $\mathcal{C}_{c}^{\infty}(X)$ by

$$
(h \cdot f)(x)=f\left(h^{-1} \cdot x\right), \quad \text { where } h \in H, f \in \mathcal{C}_{c}^{\infty}(X), x \in X,
$$

and acts on $\mathcal{D}(X)$ by

$$
\langle h \cdot T, f\rangle=\langle T, h \cdot f\rangle, \quad \text { where } T \in \mathcal{D}(X), f \in \mathcal{C}_{c}^{\infty}(X) .
$$

For a locally constant character $\eta: H \rightarrow \mathbb{C}^{\times}$, we say that a distribution $T \in$ $\mathcal{D}(X)$ is $(H, \eta)$-invariant if $h \cdot T=\eta(h) T$ for each $h \in H$. We denote by $\mathcal{D}(X)^{H, \eta}$ the space of $(H, \eta)$-invariant distributions on $X$. If $X$ is a finite dimensional space and the Fourier transform $f \mapsto \widehat{f}$ on $\mathcal{C}_{c}^{\infty}(X)$ has already been defined, for $T \in \mathcal{D}(X)$, we denote by $\widehat{T}$ its Fourier transform, which is a distribution on $X$ defined by $\widehat{T}(f)=T(\widehat{f})$.

Fourier transforms Let $\mathbf{G}$ be a reductive group, $\mathfrak{g}$ its Lie algebra. Fix a nondegenerate symmetric bilinear form $\langle$,$\rangle on \mathfrak{g}(F)$, which is invariant under conjugation. For each subspace $\mathfrak{f}$ of $\mathfrak{g}(F)$ on which the restriction of $\langle$,$\rangle is$ nondegenerate, we always equip this subspace with the self-dual Haar measure with respect to the bi-character $\psi(\langle\rangle$,$) . Define the Fourier transform f \mapsto \widehat{f}$ on $\mathcal{C}_{c}^{\infty}(\mathfrak{f})$ by

$$
\widehat{f}(X)=\int_{\mathfrak{f}} f(Y) \psi(\langle X, Y\rangle) \mathrm{d} Y .
$$

Then $\hat{\hat{f}}(X)=f(-X)$. 
Weil index At last, we recall the definition of Weil index $\gamma_{\psi}$ associated to a quadratic space. Let $q$ be a nondegenerate quadratic form on a finite dimensional vector space $V$ over $F$. If $L \subset V$ is an $\mathcal{O}_{F}$-lattice, set $i(L)=\int_{L} \psi(q(v) / 2) \mathrm{d} v$ and $\widetilde{L}=\{v \in V: \forall \ell \in L, \psi(q(v, \ell))=1\}$. It is well known that, if $\widetilde{L} \subset 2 L$, then $|i(L)|=\operatorname{vol}(L)^{\frac{1}{2}} \operatorname{vol}(\widetilde{L})^{\frac{1}{2}}$, and $i(L)|i(L)|^{-1}$ is independent of $L$. We denote by $\gamma_{\psi}(q)$ the value $i(L)|i(L)|^{-1}$, assuming $\widetilde{L} \subset 2 L$. Recall that $\gamma_{\psi}(q)$ is an 8 th root of unity.

\section{Symmetric pairs I: general cases}

In this section, we recall some basic theory and necessary results for general symmetric pairs. We refer the reader to $[A G]$ and $[R R]$ for most of the contents.

\subsection{Actions of reductive groups}

Fix a reductive group $\mathbf{H}$ and an affine variety $\mathbf{X}$ with an action by $\mathbf{H}$, both defined over $F$. Write $H=\mathbf{H}(F)$ and $X=\mathbf{X}(F)$. Then the categorical quotient $\mathbf{X} / \mathbf{H}$ of $\mathbf{X}$ by $\mathbf{H}$ exists. In fact, $\mathbf{X} / \mathbf{H}=\operatorname{Spec}\left(\mathcal{O}(\mathbf{X})^{\mathbf{H}}\right)$. Let $\pi$ denote the natural maps $\mathbf{X} \rightarrow \mathbf{X} / \mathbf{H}$ and $X \rightarrow(\mathbf{X} / \mathbf{H})(F)$.

Let $x \in X$. We say that $x$ is

- $\mathbf{H}$-semisimple or $H$-semisimple if $\mathbf{H} x$ is Zariski closed in $\mathbf{X}$ (or equivalently, $H x$ is closed in $X$ for the analytic topology),

- H-regular or $H$-regular if the stabilizer $\mathbf{H}_{x}$ has minimal dimension.

We usually say semisimple or regular without mentioning $H$ if there is no confusion. Denote by $X_{\mathrm{rs}}\left(\right.$ resp. $X_{\mathrm{ss}}$ ) the set of regular semisimple (resp. semisimple) elements in $X$.

If $\mathbf{X}$ is an $F$-rational finite dimensional representation of $\mathbf{H}$, say a point $x \in X$ nilpotent if $0 \in \operatorname{cl}(H x)$. Let $\mathcal{N}$ denote the set of nilpotent elements in $X$, which is called the null-cone of $\mathbf{X}$. Note that $\mathcal{N}=\pi^{-1}(\pi(0))$.

An open subset $U \subset X$ is called saturated if there exists an open subset $V \subset(\mathbf{X} / \mathbf{H})(F)$ such that $U=\pi^{-1}(V)$.

For $x \in X$ a semisimple element, we denote by $N_{H x, x}^{X}$ the normal space of $H x$ at $x$. Then the stabilizer $H_{x}$ acts naturally on the vector space $N_{H x, x}^{X}$. We call $\left(H_{x}, N_{H x, x}^{X}\right)$ the sliced representation at $x$, or the descendent of $(H, X)$ at $x$. Then we have the following analytic Luna Slice Theorem (cf. [AG, Theorem 2.3.17]): there exist

- an open $H$-invariant neighborhood $U_{x}$ of $H x$ in $X$ with an $H$-equivariant retract $p: U_{x} \rightarrow H x$,

- and an $H_{x}$-equivariant embedding $\psi: p^{-1}(x) \hookrightarrow N_{H x, x}^{X}$ with an open saturated image such that $\psi(x)=0$.

Write $Z_{x}=p^{-1}(x)$ and $N_{x}=N_{H x, x}^{X}$. We call $\left(U_{x}, p, \psi, Z_{x}, N_{x}\right)$ an analytic Luna slice at $x$. Let $y \in p^{-1}(x)$ and $z:=\psi(y)$. Then we have (cf. [AG, Corollary 2.3.19]):

- $\left(H_{x}\right)_{z}=H_{y}$, 
- $N_{H y, y}^{X} \simeq N_{H_{x} z, z}^{N_{x}}$ as $H_{y}$ spaces,

- $y$ is $H$-semisimple if and only if $z$ is $H_{x}$-semisimple.

\subsection{Symmetric pairs}

A symmetric pair is a triple $(\mathbf{G}, \mathbf{H}, \theta)$ where $\mathbf{H} \subset \mathbf{G}$ are reductive groups, and $\theta$ is an involution of $\mathbf{G}$ such that $\mathbf{H}=\mathbf{G}^{\theta}$ is the subgroup of fixed points. For a symmetric pair $(\mathbf{G}, \mathbf{H}, \theta)$ we define an anti-involution $\iota: \mathbf{G} \rightarrow \mathbf{G}$ by $\iota(g)=\theta\left(g^{-1}\right)$. Set $\mathbf{G}^{\iota}=\{g \in \mathbf{G} ; \iota(g)=g\}$ and define a symmetrization map

$$
s: \mathbf{G} \rightarrow \mathbf{G}^{\iota}, \quad s(g)=g \iota(g) .
$$

By this symmetrization map we can view the symmetric space $S:=G / H$ as a subset of $\mathbf{G}^{\iota}(F)$. We consider the action of $\mathbf{H} \times \mathbf{H}$ on $\mathbf{G}$ by left and right translation and the action of $\mathbf{H}$ on $\mathbf{G}^{\iota}$ by conjugation.

Let $\theta$ act by its differential on $\mathfrak{g}=\operatorname{Lie}(\mathbf{G})$. Write $\mathfrak{h}=\operatorname{Lie}(\mathbf{H})$. Thus,

$$
\mathfrak{h}=\{X \in \mathfrak{g}: \theta(X)=X\} .
$$

Put

$$
\mathfrak{s}=\{X \in \mathfrak{g}: \theta(X)=-X\},
$$

on which $\mathbf{H}$ acts by adjoint action. We also call $\mathfrak{s}$ the Lie algebra of $S$ for simplicity, though, in fact $\mathfrak{s}$ is not a Lie algebra. We always write $X^{h}=h^{-1}$. $X=\operatorname{Ad}\left(h^{-1}\right) X$ for $h \in \mathbf{H}$ and $X \in \mathfrak{s}$. There exists a G-invariant $\theta$-invariant nondegenerate symmetric bilinear form $\langle$,$\rangle on \mathfrak{g}$. In particular, $\mathfrak{g}=\mathfrak{h} \oplus \mathfrak{s}$ is an orthogonal direct sum with respect to $\langle$,$\rangle .$

Let $(\mathbf{G}, \mathbf{H}, \theta)$ be a symmetric pair. Let $g \in G$ be $H \times H$-semisimple, and $x=s(g)$. Then the triple $\left(\mathbf{G}_{x}, \mathbf{H}_{x},\left.\theta\right|_{\mathbf{G}_{x}}\right)$ is still a symmetric pair, and we have (cf. [AG, Proposition 7.2.1])

- $x$ is semisimple (both as an element of $G$ and with respect to the $H$-action),

- $H_{x} \simeq(H \times H)_{g}$ and $\mathfrak{s}_{x} \simeq N_{H g H, H}^{G}$ as $H_{x}$-spaces, where $\mathfrak{s}_{x}$ is the centralizer of $x$ in $\mathfrak{s}(F)$.

A symmetric pair obtained in this way is called a descendant of $(\mathbf{G}, \mathbf{H}, \theta)$. Note that $\mathfrak{s}_{x}$ can be identified with the Lie algebra of $\mathbf{G}_{x} / \mathbf{H}_{x}$.

Weyl integration formula Let $(\mathbf{G}, \mathbf{H}, \theta)$ be a symmetric pair. Denote by $\mathfrak{s}_{\mathrm{rs}}$ the regular and semisimple locus in $\mathfrak{s}$ with respect to the $\mathbf{H}$-action. We call a torus $\mathbf{T}$ of $\mathbf{G} \theta$-split if $\theta(t)=t^{-1}$ for all $t \in \mathbf{T}$. Fix a Cartan subspace $\mathfrak{c}$ of $\mathfrak{s}$, which by definition is a maximal abelian subspace of $\mathfrak{s}$ consisting of $\mathbf{H}$ semisimple elements. We always assume that a Cartan subspace is $F$-rational when we mention it. Then there is an $F$-rational $\theta$-split torus denoted by $\mathbf{T}^{-}$ whose Lie algebra is $\mathfrak{c}$. Denote by $\mathfrak{c}_{\text {reg }}$ the $\mathbf{H}$-regular locus in $\mathfrak{c}$. Let $\mathbf{T}$ be the centralizer of $\mathfrak{c}$ in $\mathbf{H}$, which is a torus. Write $\mathfrak{t}=\operatorname{Lie}(\mathbf{T})$.

For $X \in \mathfrak{c}_{\text {reg }}(F)$, we now introduce the factor $\left|D^{\mathfrak{s}}(X)\right|_{F}$. Consider the morphism

$$
\beta:(\mathbf{T} \backslash \mathbf{H}) \times \mathfrak{c} \longrightarrow \mathfrak{s}, \quad(h, X) \mapsto X^{h},
$$


which is regular at $(1, X)$. The Jacobian of the differential $\mathrm{d} \beta$ at $(1, X)$ is equal to

$$
\left|D^{\mathfrak{s}}(X)\right|_{F}:=|\operatorname{det}(\operatorname{ad}(X) ; \mathfrak{h} / \mathfrak{t} \oplus \mathfrak{s} / \mathfrak{c})|_{F}^{\frac{1}{2}} .
$$

Denote by $S_{\mathfrak{c}}$ the set of roots of $\mathbf{T}^{-}$in $\mathfrak{g}(\bar{F})$. For any $\alpha \in S_{\mathfrak{c}}$, since $\mathfrak{c} \subset \mathfrak{s}$, we have $\theta(\alpha)=-\alpha$. Therefore $\theta$ interchanges the root subspaces $\mathfrak{g}_{\alpha}$ and $\mathfrak{g}_{-\alpha}$. Fix a set of positive abstract roots in $S_{\mathfrak{c}}$, and choose a basis $\left\{E_{1}, E_{2}, \ldots, E_{k}\right\}$ of root vectors for the direct sum of $\mathfrak{g}_{\alpha}$ with $\alpha>0$. Set $\mathfrak{g}_{1}=\oplus_{\alpha \in S_{\mathrm{c}}} \mathfrak{g}_{\alpha}$ so that $\mathfrak{g}=\mathfrak{t} \oplus \mathfrak{c} \oplus \mathfrak{g}_{1}$. Then over $\bar{F}$,

- $\left\{E_{1}, E_{2}, \ldots, E_{k}\right\} \cup\left\{\theta\left(E_{1}\right), \theta\left(E_{2}\right), \ldots, \theta\left(E_{k}\right)\right\}$ is a basis for $\mathfrak{g}_{1}$;

- $\left\{E_{1}-\theta\left(E_{1}\right), E_{2}-\theta\left(E_{2}\right), \ldots, E_{k}-\theta\left(E_{k}\right)\right\}$ is a basis for $\mathfrak{s}_{1}:=\mathfrak{s} \cap \mathfrak{g}_{1}$;

- $\left\{E_{1}+\theta\left(E_{1}\right), E_{2}+\theta\left(E_{2}\right), \ldots, E_{k}+\theta\left(E_{k}\right)\right\}$ is a basis for $\mathfrak{h}_{1}:=\mathfrak{h} \cap \mathfrak{g}_{1}$.

Under the adjoint action, elements of $\mathfrak{c}$ map $\mathfrak{h}_{1}$ to $\mathfrak{s}_{1}$ and vice versa. There is an involution $\varrho$ on $\mathfrak{g}_{1}$ whose +1 -eigenspace is $\oplus_{\alpha>0} \mathfrak{g}_{\alpha}$ and whose -1-eigenspace is $\oplus_{\alpha<0} \mathfrak{g}_{\alpha}$. Then $\varrho$ interchanges $\mathfrak{s}_{1}$ and $\mathfrak{h}_{1}$, and $\varrho$ commutes with $\operatorname{ad}(X)$ for $X$ in $\mathfrak{c}(F)$. Thus we have

$$
\left|D^{\mathfrak{s}}(X)\right|_{F}=|\operatorname{det}(\varrho \circ \operatorname{ad}(X) ; \mathfrak{h} / \mathfrak{t})|_{F}=|\operatorname{det}(\varrho \circ \operatorname{ad}(X) ; \mathfrak{s} / \mathfrak{c})|_{F} .
$$

For a Cartan subspace $\mathfrak{c}$, let $M$ be its normalizer in $H, W_{\mathfrak{c}}:=M / T$ be its Weyl group. The map

$$
(T \backslash H) \times \mathfrak{c}_{\mathrm{reg}}(F) \longrightarrow \mathfrak{s}_{\mathrm{rs}}(F)
$$

obtained from $\beta$ by restriction is a local isomorphism of $p$-adic manifolds and its image, denoted by $\mathfrak{s}_{\text {rs }}^{\mathfrak{c}}$, is open in $\mathfrak{s}(F)$. The fiber of $\beta$ through $(h, X) \in$ $(T \backslash H) \times \mathfrak{c}_{\text {reg }}(F)$ has $\left|W_{\mathfrak{c}}\right|$ elements. We have

$$
\mathfrak{s}_{\mathrm{rs}}(F)=\bigsqcup_{\mathfrak{c}} \mathfrak{s}_{\mathrm{rs}}^{\mathfrak{c}},
$$

where the union runs over a (finite) set of representatives $\mathfrak{c}$ for the set of $H$ conjugacy classes of $F$-rational Cartan subspaces in $\mathfrak{s}$. Then, for $f \in \mathcal{C}_{c}^{\infty}(\mathfrak{s}(F))$, we have the following Weyl integration formula (cf. [RR, page 106])

$$
\int_{\mathfrak{s}(F)} f(X) \mathrm{d} X=\sum_{\mathfrak{c}} \frac{1}{\left|W_{\mathfrak{c}}\right|} \int_{\mathfrak{c}_{\mathrm{reg}}(F)}\left|D^{\mathfrak{s}}(X)\right|_{F} \int_{T \backslash H} f\left(X^{h}\right) \mathrm{d} h \mathrm{~d} X .
$$

The null-cone Denote by $\mathcal{N}$ the null-cone of $\mathfrak{s}(F)$ with respect to the $H$ action. Then, by $[\mathrm{AG}$, Theorem 7.3.8], $\mathcal{N}$ is also the set of nilpotent elements (considered as elements in $\mathfrak{g}$ ) in $\mathfrak{s}(F)$. It is known that $\mathcal{N}$ consists of finitely many $H$-orbits. Denote by $\mathcal{N}_{q}$ the union of all $H$-orbits in $\mathcal{N}$ of dimension $\leq q$, which is closed in $\mathcal{N}_{q+1}$.

Fix $X_{0} \neq 0$ in $\mathcal{N}$. Denote by $X_{0}^{H}$ the $H$-orbit of $X_{0}$, and $\mathfrak{h}_{X_{0}}$ the centralizer of $X_{0}$ in $\mathfrak{h}(F)$. Write $r=\operatorname{dim} \mathfrak{h}_{X_{0}}$. Then $X_{0}^{H}$ is of dimension $d-r$ where $d=\operatorname{dim} \mathfrak{h}(F)$, and is open in $\mathcal{N}_{d-r}$.

Lemma 3.1. There exists a group homomorphism $\phi: \mathrm{SL}_{2}(F) \rightarrow G$ such that

$$
\mathrm{d} \phi\left(\left(\begin{array}{ll}
0 & 1 \\
0 & 0
\end{array}\right)\right)=X_{0}, \mathrm{~d} \phi\left(\left(\begin{array}{ll}
0 & 0 \\
1 & 0
\end{array}\right)\right)=: Y_{0}, \phi\left(\left(\begin{array}{cc}
t & 0 \\
0 & t^{-1}
\end{array}\right)\right)=: \mathrm{D}_{t}\left(X_{0}\right),
$$

with $Y_{0} \in \mathfrak{s}(F)$ and $\mathrm{D}_{t}\left(X_{0}\right) \in H$. 
Proof. See [AG, Lemma 7.1.11].

We write $\mathrm{d}\left(X_{0}\right)=\mathrm{d} \phi\left(\left(\begin{array}{cc}1 & 0 \\ 0 & -1\end{array}\right)\right)$, which is in $\mathfrak{h}(F)$. Actually, we often write $\mathrm{d}=\mathrm{d}\left(X_{0}\right)$ when there is no confusion. For any $X \in \mathfrak{s}(F)$, we denote by $\mathfrak{s}_{X}\left(\right.$ resp. $\left.\mathfrak{g}_{X}\right)$ the centralizer of $X$ in $\mathfrak{s}(F)$ (resp. $\mathfrak{g}(F)$ ).

Lemma 3.2. We have

$$
\mathfrak{s}_{Y_{0}} \oplus\left[X_{0}, \mathfrak{h}(F)\right]=\mathfrak{s}(F), \quad \mathfrak{s}_{X_{0}} \oplus\left[Y_{0}, \mathfrak{h}(F)\right]=\mathfrak{s}(F) .
$$

Proof. We have the following decompositions (cf. [HC1, page 73])

$$
\mathfrak{g}_{Y_{0}} \oplus\left[X_{0}, \mathfrak{g}(F)\right]=\mathfrak{g}_{X_{0}} \oplus\left[Y_{0}, \mathfrak{g}(F)\right]=\mathfrak{g}(F) .
$$

From the decomposition

$$
\mathfrak{g}(F)=\mathfrak{h}(F) \oplus \mathfrak{s}(F)
$$

we see that

$$
\mathfrak{g}_{X_{0}}=\mathfrak{h}_{X_{0}} \oplus \mathfrak{s}_{X_{0}}, \quad \mathfrak{g}_{Y_{0}}=\mathfrak{h}_{Y_{0}} \oplus \mathfrak{s}_{Y_{0}},
$$

since

$$
\left[X_{0}, \mathfrak{h}(F)\right] \subset \mathfrak{s}(F),\left[X_{0}, \mathfrak{s}(F)\right] \subset \mathfrak{h}(F),\left[Y_{0}, \mathfrak{h}(F)\right] \subset \mathfrak{s}(F),\left[Y_{0}, \mathfrak{s}(F)\right] \subset \mathfrak{h}(F) .
$$

Thus we have

$$
\left(\mathfrak{h}_{Y_{0}} \oplus \mathfrak{s}_{Y_{0}}\right) \bigoplus\left(\left[X_{0}, \mathfrak{s}(F)\right] \oplus\left[X_{0}, \mathfrak{h}(F)\right]\right)=\mathfrak{h}(F) \oplus \mathfrak{s}(F),
$$

and

$$
\left(\mathfrak{h}_{X_{0}} \oplus \mathfrak{s}_{X_{0}}\right) \bigoplus\left(\left[Y_{0}, \mathfrak{s}(F)\right] \oplus\left[Y_{0}, \mathfrak{h}(F)\right]\right)=\mathfrak{h}(F) \oplus \mathfrak{s}(F) .
$$

Taking the $\mathfrak{s}$-parts of the above identities, we prove the assertions of the lemma.

Let $\Gamma$ be the Cartan subgroup of $H$ with the Lie algebra $F \cdot \mathrm{d}\left(X_{0}\right)$. Let $\xi$ be the rational character of $\Gamma$ defined by

$$
X_{0}^{\gamma}=\xi(\gamma) X_{0}, \quad Y_{0}^{\gamma}=\xi^{-1}(\gamma) Y_{0},
$$

which is not trivial. Let $r^{\prime}=\operatorname{dim} \mathfrak{s}_{Y_{0}}$. The following lemma essentially is a variant of [HC1, Lemma 34], and the proof is also similar to that of $[\mathrm{HC}$, Lemma 34].

Lemma 3.3. We can choose a basis $Y_{0}=U_{1}, U_{2}, \ldots, U_{r^{\prime}}$ for $\mathfrak{s}_{Y_{0}}$ and rational characters $\xi_{1}, \xi_{2}, \ldots, \xi_{r^{\prime}}$ of $\Gamma$ such that

1. $\xi_{i}^{2}=\xi^{\lambda_{i}}, \lambda_{i} \geq 0$,

2. $\operatorname{ad}(-\mathrm{d}) U_{i}=\lambda_{i} U_{i}$,

3. $U_{i}^{\gamma}=\xi_{i}^{-1}(\gamma) U_{i}$, for all $1 \leq i \leq r^{\prime}$.

Set

$$
m=\frac{1}{2}\left(\sum_{1 \leq i \leq r^{\prime}} \lambda_{i}\right)=\frac{1}{2} \operatorname{Tr}\left(\left.\operatorname{ad}(-\mathrm{d})\right|_{\mathfrak{s}_{Y_{0}}}\right) .
$$




\section{Symmetric pairs II: specific cases}

Now we focus on the symmetric pairs concerned in this article. The notations introduced here will be used without mention from now on.

\section{$4.1 \quad(\mathrm{G}, \mathrm{H})$}

Let $\mathbf{G}=\mathrm{GL}_{2 n}$ and $\mathbf{H}=\mathrm{GL}_{n} \times \mathrm{GL}_{n}$, both defined over $F$. $\mathbf{H}$ is viewed as a subgroup of $\mathbf{G}$ by embedding it into $\mathbf{G}$ diagonally. Let $\epsilon=\left(\begin{array}{cc}\mathbf{1}_{n} & 0 \\ 0 & -\mathbf{1}_{n}\end{array}\right)$ and define an involution $\theta$ on $\mathbf{G}$ by $\theta(g)=\epsilon g \epsilon$. Then $\mathbf{H}=\mathbf{G}^{\theta}$, and the Lie algebra $\mathfrak{s}$ associated to $(\mathbf{G}, \mathbf{H}, \theta)$ is

$$
\mathfrak{s}(F)=\left\{\left(\begin{array}{cc}
0 & A \\
B & 0
\end{array}\right): A, B \in \mathfrak{g l}_{n}(F)\right\} \simeq \mathfrak{g l}_{n}(F) \oplus \mathfrak{g l}_{n}(F) .
$$

If we identify $\mathfrak{s}(F)$ with $\mathfrak{g l}_{n}(F) \oplus \mathfrak{g l}_{n}(F)$, then $H$ acts on $\mathfrak{s}(F)$ by

$$
\left(h_{1}, h_{2}\right) \cdot(A, B)=\left(h_{1} A h_{2}^{-1}, h_{2} B h_{1}^{-1}\right) .
$$

Recall that we write $X^{h}=h^{-1} \cdot X$ for $h \in H, X \in \mathfrak{s}(F)$. We fix a nondegenerate symmetric bilinear form $\langle$,$\rangle on \mathfrak{g}(F)$ defined by

$$
\langle X, Y\rangle=\operatorname{tr}(X Y), \quad \text { for } X, Y \in \mathfrak{g}(F) .
$$

Then $\langle$,$\rangle is both G$-invariant and $\theta$-invariant.

Since $H^{1}(F, \mathbf{H})$ is trivial, we have $S=\mathcal{S}(F)$ where $S:=G / H$ and $\mathcal{S}:=$ $\mathbf{G} / \mathbf{H}$. We identify $S$ with its image in $\mathbf{G}^{\iota}(F)$ by the the symmetrization map $s$. When we want to emphasize the index $n$, we write $\mathbf{G}_{n}, \mathbf{H}_{n}, \theta_{n}$ and $\mathfrak{s}_{n}$.

Descendants Now we describe all the $H$-semisimple elements $x$ of $S$ and $\mathfrak{s}(F)$ and the descendants $\left(H_{x}, \mathfrak{s}_{x}\right)$ at $x$. The results below also hold when $F=k$ is a number field.

Proposition 4.1. 1. Each semisimple element $x$ of $S$ is $H$-conjugate to an element of the form

$$
x\left(A, n_{1}, n_{2}\right):=\left(\begin{array}{cccccc}
A & 0 & 0 & A-\mathbf{1}_{m} & 0 & 0 \\
0 & \mathbf{1}_{n_{1}} & 0 & 0 & 0 & 0 \\
0 & 0 & -\mathbf{1}_{n_{2}} & 0 & 0 & 0 \\
A+\mathbf{1}_{m} & 0 & 0 & A & 0 & 0 \\
0 & 0 & 0 & 0 & \mathbf{1}_{n_{1}} & 0 \\
0 & 0 & 0 & 0 & 0 & -\mathbf{1}_{n_{2}}
\end{array}\right),
$$

with $n=m+n_{1}+n_{2}, A \in \mathfrak{g l}_{m}(F)$ being semisimple without eigenvalues \pm 1 and unique up to conjugation. Moreover, $x\left(A, n_{1}, n_{2}\right)$ is regular if and only if $n_{1}=n_{2}=0$ and $A$ is regular in $\mathfrak{g l}_{n}(F)$.

2. Let $x=x\left(A, n_{1}, n_{2}\right)$ in $S$ be semisimple. Then the descendant $\left(H_{x}, \mathfrak{s}_{x}\right)$ is isomorphic to the product (as a representation)

$$
\left(\mathrm{GL}_{m}(F)_{A}, \mathfrak{g l}_{m}(F)_{A}\right) \times\left(H_{n_{1}}, \mathfrak{s}_{n_{1}}\right) \times\left(H_{n_{2}}, \mathfrak{s}_{n_{2}}\right) .
$$

Here $\mathrm{GL}_{m}(F)_{A}$ and $\mathfrak{g l}_{m}(F)_{A}$ are the centralizers of $A$ in $\mathrm{GL}_{m}(F)$ and $\mathfrak{g l}_{m}(F)$ respectively, and $\mathrm{GL}_{m}(F)_{A}$ acts on $\mathfrak{g l}_{m}(F)_{A}$ by conjugation. 
Proof. See [JR, Proposition 4.1] or [Gu1, Proposition 1.1] for the first assertion. The second assertion can be proved by a direct computation.

Proposition 4.2. 1. Each semisimple element $X$ of $\mathfrak{s}(F)$ is $H$-conjugate to an element of the form

$$
X(A)=\left(\begin{array}{cccc}
0 & 0 & \mathbf{1}_{m} & 0 \\
0 & 0 & 0 & 0 \\
A & 0 & 0 & 0 \\
0 & 0 & 0 & 0
\end{array}\right)
$$

with $A \in \mathrm{GL}_{m}(F)$ being semisimple and unique up to conjugation. Moreover, $X(A)$ is regular if and only if $m=n$ and $A \in \mathrm{GL}_{n}(F)$ is regular.

2. Let $X=X(A)$ in $\mathfrak{s}(F)$ be semisimple. Then the descendant $\left(H_{X}, \mathfrak{s}_{X}\right)$ is isomorphic to the product (as a representation)

$$
\left(\mathrm{GL}_{m}(F)_{A}, \mathfrak{g l}_{m}(F)_{A}\right) \times\left(H_{n-m}, \mathfrak{s}_{n-m}\right) .
$$

Proof. See [JR, Propositions 2.1 and 2.2].

The null-cone Fix $X_{0} \neq 0$ in the null-cone $\mathcal{N}$ of $\mathfrak{s}(F)$. Let $\left(X_{0}, \mathrm{~d}, Y_{0}\right)$ be an $\mathfrak{s l}_{2}$-triple as before. Recall $\mathrm{d}=\mathrm{d}\left(X_{0}\right)$.

Lemma 4.3. We have $\operatorname{dim} \mathfrak{s}_{Y_{0}}=\operatorname{dim} \mathfrak{h}_{X_{0}}=r$.

Proof. It follows from Lemma 3.2 and the relation

$$
\operatorname{dim} \mathfrak{h}_{X_{0}}+\operatorname{dim}\left[X_{0}, \mathfrak{h}(F)\right]=\operatorname{dim} \mathfrak{h}(F)=\operatorname{dim} \mathfrak{s}(F) .
$$

In [JR, Lemma 3.1], $\mathfrak{h}_{X_{0}}$ is well studied, and an upper bound for $\operatorname{Tr}\left(\left.\operatorname{ad}(d)\right|_{\mathfrak{h}_{X_{0}}}\right)$ is given there. By a minor modification of the discussion in [JR, §3], we study the structure of $\mathfrak{s}_{Y_{0}}$. For our purpose, we want to compare $r+m$ with $n^{2}+\frac{n}{2}$, where $r=\operatorname{dim} \mathfrak{s}_{Y_{0}}$ and $m=\frac{1}{2} \operatorname{Tr}\left(\left.\operatorname{ad}(-\mathrm{d})\right|_{\mathfrak{s}_{Y_{0}}}\right)$. The following inequalities will be used in $\S 6.3$.

Proposition 4.4. We have the relations

$$
\begin{aligned}
& \text { 1. } r \geq n, \\
& \text { 2. } r+m>n^{2}+\frac{n}{2} \text {. }
\end{aligned}
$$

Proof. Write $Y=Y_{0}$ for short. Let $V=V_{0} \oplus V_{1}$, where $V_{i}=F^{n}, 0 \leq i \leq$ 1. We identify $\mathfrak{g}(F)=\operatorname{Hom}(V, V), \mathfrak{h}(F)=\operatorname{Hom}\left(V_{0}, V_{0}\right) \oplus \operatorname{Hom}\left(V_{1}, V_{1}\right)$ and $\mathfrak{s}(F)=\operatorname{Hom}\left(V_{1}, V_{0}\right) \oplus \operatorname{Hom}\left(V_{0}, V_{1}\right)$. Given $Y$, there is a decomposition $V=$ $W_{1} \oplus W_{2} \oplus \cdots \oplus W_{k}$, where each $W_{i}$ is an indecomposable $F[Y]$-submodule. We can choose a generator $z_{i}$ of $W_{i}$ such that $z_{i}$ is in either $V_{0}$ or $V_{1}$. Define $\operatorname{deg}\left(z_{i}\right)=0$ if $z_{i} \in V_{0}$, otherwise $\operatorname{deg}\left(z_{i}\right)=1$. Write $w_{i}=\operatorname{dim} W_{i}$. There is an isomorphism from $\mathfrak{s}_{Y_{0}}$ to some space

$$
\mathcal{Z}=\oplus_{1 \leq i, j \leq k} S_{i j}
$$

Now we describe $S_{i j}$ precisely. An element $b_{i j} \in S_{i j}$ is in $F[X] /\left(X^{w_{j}}\right)$ of the form: 
1. $b_{i j}(X)=\sum_{\max \left\{w_{j}-w_{i}, 0\right\} \leq \ell<w_{j}} a_{\ell}^{i j} X^{\ell}$,

2. $a_{\ell}^{i j}=0$ when $\delta_{i} \delta_{j}=(-1)^{\ell}$, where $\delta_{i}:=(-1)^{\operatorname{deg}\left(z_{i}\right)}$.

We define an operator $\rho(\mathrm{d}):=X \frac{\mathrm{d}}{\mathrm{d} X}$ on $F[X]$, and an endomorphism $\rho(\mathrm{d})$ on $\mathcal{Z}$ by restriction. Each $S_{i j}$ is an invariant subspace of $\rho(\mathrm{d})$. Set

$$
r_{i i}=\operatorname{dim} S_{i i}, \quad m_{i i}=\operatorname{Tr}\left(\left.\rho(\mathrm{d})\right|_{S_{i i}}\right)
$$

for $1 \leq i \leq k$, and

$$
r_{i j}=\operatorname{dim} S_{i j}+\operatorname{dim} S_{j i}, \quad m_{i j}=\operatorname{Tr}\left(\left.\rho(\mathrm{d})\right|_{S_{i j}}\right)+\operatorname{Tr}\left(\left.\rho(\mathrm{d})\right|_{S_{i j}}\right)
$$

for $1 \leq i<j \leq k$. Then

$$
r=\sum_{1 \leq i \leq k} r_{i i}+\sum_{1 \leq i<j \leq k} r_{i j}, \quad m=\sum_{1 \leq i \leq k} m_{i i}+\sum_{1 \leq i<j \leq k} m_{i j} .
$$

The following lemma gives a complete list of $r_{i i}, r_{i j}, m_{i i}$ and $m_{i j}$. One can obtain it by the above description and a direct computation.

Lemma 4.5. 1. For $1 \leq i \leq k$, if $w_{i}=2 p_{i}$ or $2 p_{i}+1$, we have

$$
r_{i i}=p_{i}, \quad m_{i i}=p_{i}^{2} .
$$

2. For $1 \leq i<j \leq k$, we have the following table.

\begin{tabular}{|c|c|c|c|}
\hline$w_{i}, w_{j}$ & $\delta_{i} \delta_{j}$ & $m_{i j}$ & $r_{i j}$ \\
\hline$w_{i}=2 p_{i}, w_{j}=2 p_{j}$ & 1 & $2 p_{i} p_{j}$ & $2 \min \left(p_{i}, p_{j}\right)$ \\
\hline$w_{i}=2 p_{i}, w_{j}=2 p_{j}$ & -1 & $2 p_{i} p_{j}-2 \min \left(p_{i}, p_{j}\right)$ & $2 \min \left(p_{i}, p_{j}\right)$ \\
\hline$w_{i}=2 p_{i}, w_{j}=2 p_{j}+1, w_{i}<w_{j}$ & \pm 1 & $2 p_{i} p_{j}$ & $2 p_{i}$ \\
\hline$w_{i}=2 p_{i}, w_{j}=2 p_{j}+1, w_{i}>w_{j}$ & 1 & $2 p_{i} p_{j}+2\left(p_{i}-p_{j}\right)-1$ & $2 p_{j}+1$ \\
\hline$w_{i}=2 p_{i}, w_{j}=2 p_{j}+1, w_{i}>w_{j}$ & -1 & $2 p_{i} p_{j}$ & $2 p_{j}+1$ \\
\hline$w_{i}=2 p_{i}+1, w_{j}=2 p_{j}+1$ & 1 & $2 p_{i} p_{j}$ & $2 \min \left(p_{i}, p_{j}\right)$ \\
\hline$w_{i}=2 p_{i}+1, w_{j}=2 p_{j}+1$ & -1 & $2 p_{i} p_{j}+2 \sup \left(p_{i}, p_{j}\right)$ & $2 \min \left(p_{i}, p_{j}\right)+2$ \\
\hline
\end{tabular}

Now we continue to prove the proposition.

(1) The first inequality of the proposition can be read off from the above list. It is not hard to see that $r=n$ if and only if $Y^{2 n}=0$ and $Y^{2 n-1} \neq 0$.

(2) For the second inequality, compare with the proof of [JR, Lemma 3.1]. We denote by $u$ the number of indices $i$ such that $w_{i}$ is odd and $\delta_{i}=1$, which is equal to the number of indices $j$ such that $w_{j}$ is odd and $\delta_{j}=-1$. Then

$$
n=u+\sum_{1 \leq i \leq k} p_{i}
$$

where $w_{i}=2 p_{i}$ or $2 p_{i}+1$. Thus

$$
n^{2}+\frac{n}{2}=u^{2}+\frac{u}{2}+\left(2 u+\frac{1}{2}\right)\left(\sum_{1 \leq i \leq k} p_{i}\right)+\sum_{1 \leq i \leq k} p_{i}^{2}+2 \sum_{1 \leq i<j \leq k} p_{i} p_{j} .
$$


On the other hand

$$
r+m=\sum_{1 \leq i \leq k}\left(r_{i i}+m_{i i}\right)+\sum_{1 \leq i<j \leq k}\left(r_{i j}+m_{i j}\right)
$$

is determined by the data

$$
\left(w_{1}, \delta_{1}, w_{2}, \delta_{2}, \ldots, w_{k}, \delta_{k}\right) .
$$

We now induct on the number of indices $i$ so that $w_{i}$ is even. First assume all the integers $w_{i}$ are odd. Then it is not hard to see that

$$
\begin{aligned}
r+m= & \sum_{1 \leq i \leq k}\left(p_{i}^{2}+p_{i}\right)+2 \sum_{1 \leq i<j \leq k} p_{i} p_{j}+2 \sum_{1 \leq i<j \leq k, \delta_{i} \delta_{j}=1} \min \left(p_{i}, p_{j}\right) \\
& +2 \sum_{1 \leq i<j \leq k, \delta_{i} \delta_{j}=-1}\left(\sup \left(p_{i}, p_{j}\right)+\min \left(p_{i}, p_{j}\right)+1\right) \\
= & \sum_{1 \leq i \leq k}\left(p_{i}^{2}+p_{i}\right)+2 \sum_{1 \leq i<j \leq k} p_{i} p_{j}+2 \sum_{1 \leq i<j \leq k, \delta_{i} \delta_{j}=1} \min \left(p_{i}, p_{j}\right) \\
& +2 u \sum_{1 \leq i \leq k} p_{i}+2 u^{2} \\
\geq & 2 u^{2}+(2 u+1)\left(\sum_{1 \leq i \leq k} p_{i}\right)+\sum_{1 \leq i \leq k} p_{i}^{2}+2 \sum_{1 \leq i<j \leq k} p_{i} p_{j} \\
> & n^{2}+\frac{n}{2} .
\end{aligned}
$$

Now we can arrange the data so that $w_{k}$ is even. If $k=1$, then $w_{1}=2 n$ and $r+m=n^{2}+n$ which is strictly greater than $n^{2}+\frac{n}{2}$. By induction on the number of indices $i$ with $w_{i}$ even, we may assume that the inequality has been proved for the data $\left(w_{1}, \delta_{1}, \ldots, w_{k-1}, \delta_{k-1}\right)$. By the induction hypothesis, the contribution of the indices $(i, j)$ with $1 \leq i \leq j \leq k-1$ is strictly greater than $n^{\prime 2}+\frac{n^{\prime}}{2}$ where

$$
n^{\prime}=u+\sum_{1 \leq i<k} p_{i} .
$$

Therefore we have to show that the sum of the contributions $r_{i k}+m_{i k}$ of the pairs $(i, k)$ with $i \leq k$ is greater than or equal to

$$
n^{2}+\frac{n}{2}-n^{\prime 2}-\frac{n^{\prime}}{2}=p_{k}^{2}+\frac{p_{k}}{2}+2 \sum_{1 \leq i<k} p_{i} p_{k}+2 u p_{k} .
$$

The contribution of the pair $(k, k)$ is $p_{k}^{2}+p_{k}>p_{k}^{2}+\frac{p_{k}}{2}$. Now consider the contribution of a pair $(i, k)$ with $i<k$. It is always greater than or equal to $2 p_{i} p_{k}$ when $w_{i}=2 p_{i}$. When $w_{i}=2 p_{i}+1$, it is always greater than or equal to $2 p_{i} p_{k}+2 p_{k}$ (called good case) except when $w_{k}>w_{i}$ and $\delta_{i} \delta_{k}=-1$ (called bad case). It contributes at least $2 p_{i} p_{k}$ in bad case. However it does not matter when bad cases happen. Since if bad cases happen $u^{\prime}$ times with $\delta_{i}=-\delta_{k}$, good cases happen at least $u^{\prime}$ times with $w_{j}$ such that $w_{j}=2 p_{j}+1$ and $\delta_{j}=\delta_{k}$, which contribute at least $2 u^{\prime} p_{k}+\sum_{j} 2 p_{j} p_{k}$. This concludes the proof of the proposition. 


\section{$4.2\left(\mathbf{G}^{\prime}, \mathbf{H}^{\prime}\right)$}

Let $E=F(\sqrt{\Delta})$ be a quadratic extension field of $F$, D a quaternion algebra over $F$ containing $E$. Let $\eta$ be the quadratic character of $F^{\times}$associated to $E$ by the local class field theory. Denote by $\sigma$ the nontrivial element in $\operatorname{Gal}(E / F)$. Sometimes we also write $x \mapsto \bar{x}$ instead of $x \mapsto \sigma(x)$. Let $\mathbf{G}^{\prime}=\mathrm{GL}_{n}(\mathrm{D})$, $\mathbf{H}^{\prime}=\mathrm{GL}_{n}(E)$, both viewed as reductive groups defined over $F$. We can write $\mathbf{G}^{\prime}$ and $\mathbf{H}^{\prime}$ in a more concrete form. There is a $\gamma \in F^{\times}$such that, if we denote by $\mathrm{L}_{\gamma}$ the algebra

$$
\left\{\left(\begin{array}{cc}
A & \gamma B \\
\bar{B} & \bar{A}
\end{array}\right): A, B \in \mathfrak{g l}_{n}(E)\right\},
$$

then $G^{\prime}=\mathbf{G}^{\prime}(F)=\mathrm{L}_{\gamma}^{\times}$and $H^{\prime}=\mathbf{H}^{\prime}(F)$ consists of the ones with $B=0$. We will always consider $\mathbf{G}^{\prime}$ and $\mathbf{H}^{\prime}$ in such a form. Note that if $\gamma \in \mathrm{N}_{E / F} E^{\times}$, then $\mathbf{G}^{\prime} \simeq \mathrm{GL}_{2 n}$. Fix a square root $\delta$ of $\Delta$ in $E$. Let $\epsilon^{\prime}=\left(\begin{array}{cc}\delta \mathbf{1}_{n} & 0 \\ 0 & -\delta \mathbf{1}_{n}\end{array}\right)$. Define an involution $\theta$ on $\mathbf{G}^{\prime}$ by $\theta(g)=\epsilon^{\prime} g \epsilon^{\prime-1}$. Then $\mathbf{H}^{\prime}=\mathbf{G}^{\prime \theta}$. Let $\mathfrak{g}^{\prime}=\operatorname{Lie}\left(\mathbf{G}^{\prime}\right), \mathfrak{h}^{\prime}=$ Lie $\left(\mathbf{H}^{\prime}\right)$, and $\mathfrak{s}^{\prime}$ be the Lie algebra associated to the symmetric pair $\left(\mathbf{G}^{\prime}, \mathbf{H}^{\prime}, \theta\right)$ so that $\mathfrak{g}^{\prime}=\mathfrak{h}^{\prime} \oplus \mathfrak{s}^{\prime}$. Thus, $\mathfrak{s}^{\prime}(F)$ is the space

$$
\left\{\left(\begin{array}{cc}
0 & \gamma B \\
\bar{B} & 0
\end{array}\right): B \in \mathfrak{g l}_{n}(E)\right\} \simeq \mathfrak{g l}_{n}(E) .
$$

If we identify $\mathfrak{s}^{\prime}(F)$ with $\mathfrak{g l}_{n}(E)$, then $H^{\prime}=\mathrm{GL}_{n}(E)$ acts on $\mathfrak{s}^{\prime}(F)$ by $h \cdot X=$ $h X \bar{h}^{-1}$, which is the $\sigma$-twisted conjugation. We fix a nondegenerate symmetric bilinear form $\langle$,$\rangle on \mathfrak{g}^{\prime}(F)$ defined by

$$
\langle X, Y\rangle=\operatorname{tr}(X Y), \quad X, Y \in \mathfrak{g}^{\prime}(F) .
$$

By definition, it is easy to see $\langle X, Y\rangle \in F$, and $\langle$,$\rangle is both G^{\prime}$-invariant and $\theta$-invariant.

Since $H^{1}\left(F, \mathbf{H}^{\prime}\right)$ is trivial, we have $S^{\prime}=\mathcal{S}^{\prime}(F)$, where $S^{\prime}:=G^{\prime} / H^{\prime}$ and $\mathcal{S}^{\prime}:=\mathbf{G}^{\prime} / \mathbf{H}^{\prime}$. We identity $S^{\prime}$ with its image in $\mathbf{G}^{\prime \prime}(F)$ by the symmetrization map $s$. When we want to emphasize the index $n$, we write $\mathbf{G}_{n}^{\prime}, \mathbf{H}_{n}^{\prime}, \theta_{n}$ and $\mathfrak{s}_{n}^{\prime}$.

Before we continue, we recall some basic facts about the norm map in theory of base change. If $x \in \mathrm{GL}_{n}(E)$, we write $\mathrm{N}(x)=x \bar{x}$, which is called the norm of $x$. If $x \in \mathrm{GL}_{n}(E), \mathrm{N}(x)$ is conjugate in $\mathrm{GL}_{n}(E)$ to an element $y$ of $\mathrm{GL}_{n}(F)$, and $y$ is uniquely determined modulo conjugation in $\mathrm{GL}_{n}(F)$. We denote by $\mathrm{N}\left(\mathrm{GL}_{n}(E)\right)$ the subset of elements $y$ in $\mathrm{GL}_{n}(F)$ satisfying that there exists $x \in \mathrm{GL}_{n}(E)$ such that $y$ is conjugate to $\mathrm{N}(x)$. In fact, if $y \in \mathrm{N}\left(\mathrm{GL}_{n}(E)\right)$, there exists $x \in \mathrm{GL}_{n}(E)$ such that $y=x \bar{x}$.

Descendants Now we describe all the $H^{\prime}$-semisimple elements $x$ of $S^{\prime}$ and $\mathfrak{s}^{\prime}(F)$ and the descendants at $x$. The results below also hold when $F=k$ is a number field.

Proposition 4.6. 1. Each semisimple elements y of $S^{\prime}$ is $H^{\prime}$-conjugate to 
an element of the form

$$
y\left(A, n_{1}, n_{2}\right)=\left(\begin{array}{cccccc}
A & 0 & 0 & \gamma B & 0 & 0 \\
0 & \mathbf{1}_{n_{1}} & 0 & 0 & 0 & 0 \\
0 & 0 & -\mathbf{1}_{n_{2}} & 0 & 0 & 0 \\
\bar{B} & 0 & 0 & A & 0 & 0 \\
0 & 0 & 0 & 0 & \mathbf{1}_{n_{1}} & 0 \\
0 & 0 & 0 & 0 & 0 & -\mathbf{1}_{n_{2}}
\end{array}\right),
$$

with $A \in \mathfrak{g l}_{m}(F)$ being semisimple and unique up to conjugation such that $A^{2}-\mathbf{1}_{m} \in \gamma \mathrm{N}\left(\mathrm{GL}_{m}(E)\right)$ and $B \in \mathrm{GL}_{m}(E)$ is a matrix unique up to twisted conjugation such that $A^{2}-\mathbf{1}_{m}=\gamma B \bar{B}, A B=B A$, and $n=m+n_{1}+n_{2}$. Moreover, $y\left(A, n_{1}, n_{2}\right)$ is regular if and only if $n_{1}=n_{2}=0$ and $A$ is regular in $\mathfrak{g l}_{n}(F)$.

2. Let $y=y\left(A, n_{1}, n_{2}\right)$ in $S^{\prime}$ be semisimple. Then the descendant $\left(H_{y}^{\prime}, \mathfrak{s}_{y}^{\prime}\right)$ is isomorphic to the product (as a representation)

$\left(\mathrm{GL}_{m}(E)_{A} \cap \mathrm{GL}_{\sigma, m}(E)_{B}, \mathfrak{g l}_{m}(E)_{A} \cap \mathfrak{g l}_{m}^{\sigma}(E)_{B}\right) \times\left(H_{n_{1}}^{\prime}, \mathfrak{s}_{n_{1}}^{\prime}\right) \times\left(H_{n_{2}}^{\prime}, \mathfrak{s}_{n_{2}}^{\prime}\right)$.

Here

$$
\begin{gathered}
\mathrm{GL}_{\sigma, m}(E)_{B}:=\left\{h \in \mathrm{GL}_{m}(E): h B=B \bar{h}\right\}, \\
\mathfrak{g l}_{m}^{\sigma}(E)_{B}:=\left\{X \in \mathfrak{g l}_{m}(E): X \bar{B}=B \bar{X}\right\},
\end{gathered}
$$

and $\mathrm{GL}_{\sigma, m}(E)_{B}$ acts on $\mathfrak{g l}_{m}^{\sigma}(E)_{B}$ by $\sigma$-twisted conjugation.

Proof. See [Gu1, Proposition 1.2] for the first assertion. The second assertion can be proved by a direct computation.

Proposition 4.7. 1. Each semisimple element $Y$ of $\mathfrak{s}^{\prime}(F)$ is $H^{\prime}$-conjugate to an element of the form

$$
Y(A)=\left(\begin{array}{cccc}
0 & 0 & \gamma B & 0 \\
0 & 0 & 0 & 0 \\
\bar{B} & 0 & 0 & 0 \\
0 & 0 & 0 & 0
\end{array}\right)
$$

where $A \in \mathrm{GL}_{m}(F)$ is semisimple and unique up to conjugation such that $A \in \gamma \mathrm{N}\left(\mathrm{GL}_{m}(E)\right)$ and $B \in \mathrm{GL}_{m}(E)$ is a matrix unique up to twisted conjugation such that $A=\gamma B \bar{B}$. Moreover, $Y(A)$ is regular if and only if $A \in \mathrm{GL}_{n}(F)$ is regular.

2. Let $Y=Y(A)$ in $\mathfrak{s}^{\prime}(F)$ be semisimple. Then the descendant $\left(H_{Y}^{\prime}, \mathfrak{s}_{Y}^{\prime}\right)$ is isomorphic to the product (as a representation)

$$
\left(\mathrm{GL}_{\sigma, m}(E)_{B}, \mathfrak{g l}_{m}^{\sigma}(E)_{B}\right) \times\left(H_{n-m}^{\prime}, \mathfrak{s}_{n-m}^{\prime}\right)
$$

Proof. See [Gu2, Lemma 2.1] for the first assertion. The second assertion can be proved by a direct computation. 
The null-cone Fix $X_{0} \neq 0$ in the null-cone $\mathcal{N}^{\prime}$ of $\mathfrak{s}^{\prime}(F)$. Let $\left(X_{0}, \mathrm{~d}, Y_{0}\right)$ be an $\mathfrak{s l}_{2}$-triple as before. By the same proof as Lemma 4.3 , we have

$$
\operatorname{dim} \mathfrak{s}_{Y_{0}}^{\prime}=\operatorname{dim} \mathfrak{h}_{X_{0}}^{\prime} .
$$

Write $r=\operatorname{dim} \mathfrak{s}_{Y_{0}}^{\prime}$ and $m=\frac{1}{2} \operatorname{Tr}\left(\left.\operatorname{ad}(-\mathrm{d})\right|_{\mathfrak{s}_{Y_{0}}^{\prime}}\right)$. We still want to compare $r+m$ with $n^{2}+\frac{n}{2}$, which is easier in this case.

Proposition 4.8. We have $r+m>n^{2}+\frac{n}{2}$ and $m^{\prime}<n^{2}$ where $m^{\prime}=$ $\frac{1}{2} \operatorname{Tr}\left(\left.\operatorname{ad}(-\mathrm{d})\right|_{\mathfrak{h}_{Y_{0}}^{\prime}}\right)$.

Proof. Write $Y_{0}=\left(\begin{array}{cc}0 & \gamma A \\ \bar{A} & 0\end{array}\right)$. If we change $\left(X_{0}, \mathrm{~d}, Y_{0}\right)$ to be any triple in the $H^{\prime}$-orbit of $\left(X_{0}, \mathrm{~d}, Y_{0}\right)$, the numbers $r$ and $m$ are unchanged. By [Gu2, Lemma 2.2 , we can choose $A$ to be of the Jordan normal form. At the same time, we can also choose d to be in $\mathfrak{g l}_{n}(F)$. In such situation, it is easy to see that there is a d-equivariant isomorphism $\mathfrak{s}_{Y_{0}}^{\prime} \simeq \mathfrak{h}_{Y_{0}}^{\prime}$. Thus $r=r^{\prime}$ and $m=m^{\prime}$, where $r^{\prime}=\operatorname{dim} \mathfrak{h}_{Y_{0}}^{\prime}$. Since $\mathfrak{g}_{Y_{0}}^{\prime}=\mathfrak{h}_{Y_{0}}^{\prime} \oplus \mathfrak{s}_{Y_{0}}^{\prime}$, we have $m+m^{\prime}=\frac{1}{2}\left(4 n^{2}-r-r^{\prime}\right)$. Thus we get $m=\frac{1}{4}\left(4 n^{2}-2 r\right)$ and $r+m=n^{2}+\frac{r}{2}$. The inequality $r \geq 2 n$ implies the lemma.

\section{Smooth transfer}

In this section, we introduce the main object of this article: the smooth transfer between Schwartz functions on different symmetric spaces. By several reduction steps, we explain why Theorem 5.16 implies Theorem 5.13 in details.

\subsection{Definitions}

Matching of orbits We first recall the matching between semisimple orbits in symmetric spaces $S$ and $S^{\prime}$, and then give the definition of matching between semisimple orbits in Lie algebras $\mathfrak{s}(F)$ and $\mathfrak{s}^{\prime}(F)$. These definitions of matching orbits also hold when $F=k$ is a number field.

Proposition 5.1. 1. For each semisimple element $y$ of $S^{\prime}$, there exists $h \in$ $\mathbf{H}(E)$ such that hy $h^{-1}$ belongs to $S$. This establishes an injection of the $H^{\prime}$-semisimple orbits in $S^{\prime}$ into the $H$-semisimple orbits in $S$, which carries the orbit of $y\left(A, n_{1}, n_{2}\right)$ in $S^{\prime}$ to the orbit of $x\left(A, n_{1}, n_{2}\right)$ in $S$.

2. For each semisimple element $Y$ of $\mathfrak{s}^{\prime}(F)$, there exists $h \in \mathbf{H}(E)$ such that $h Y h^{-1}$ belongs to $\mathfrak{s}(F)$. This establishes an injection of the $H^{\prime}$-semisimple orbits in $\mathfrak{s}^{\prime}(F)$ into the $H$-semisimple orbits in $\mathfrak{s}(F)$, which carries the orbit of $Y(A)$ in $\mathfrak{s}^{\prime}(F)$ to the orbit of $X(A)$ in $\mathfrak{s}(F)$.

Proof. See [Gu1, Proposition 1.3] for the first assertion. The second assertion can be proved in the same way.

Definition 5.2. 1 . We say that $y \in S_{\mathrm{ss}}^{\prime}\left(\right.$ resp. $\left.Y \in \mathfrak{s}_{\mathrm{ss}}^{\prime}(F)\right)$ matches $x \in S_{\mathrm{ss}}$ (resp. $X \in \mathfrak{s}_{\mathrm{ss}}(F)$ ) and write $x \leftrightarrow y$ (resp. $X \leftrightarrow Y$ ) if the above map sends the orbit of $y$ (resp. $Y$ ) to the orbit of $x$ (resp. $X)$. 
2. We say that $x \in S_{\mathrm{ss}}$ (resp. $\left.X \in \mathfrak{s}_{\mathrm{Ss}}(F)\right)$ comes from $S_{\mathrm{ss}}^{\prime}\left(\operatorname{resp} . \mathfrak{s}_{\mathrm{sS}}^{\prime}(F)\right)$ if there exists $y \in S_{\mathrm{SS}}^{\prime}\left(\right.$ resp. $\left.Y \in \mathfrak{s}_{\mathrm{sS}}^{\prime}(F)\right)$ such that $x \leftrightarrow y(\operatorname{resp} . X \leftrightarrow Y)$. We denote by $S_{\mathrm{ss}, 0}$ (resp. $\left.\mathfrak{s}_{\mathrm{ss}}(F)_{0}\right)$ the subset of elements in $S_{\mathrm{ss}}$ (resp. $\left.\mathfrak{s}_{\mathrm{ss}}(F)\right)$ coming from $S_{\mathrm{ss}}^{\prime}\left(\right.$ resp. $\left.\mathfrak{s}_{\mathrm{ss}}^{\prime}(F)\right)$.

Remark 5.3. Denote by $\mathcal{Q}$ (resp. $\mathcal{Q}^{\prime}$ ) the categorical quotient $\mathcal{S} / \mathbf{H}$ (resp. $\mathcal{S}^{\prime} / \mathbf{H}^{\prime}$ ), and by $\mathfrak{q}\left(\right.$ resp. $\left.\mathfrak{q}^{\prime}\right)$ the categorical quotient $\mathfrak{s} / \mathbf{H}\left(\right.$ resp. $\left.\mathfrak{s}^{\prime} / \mathbf{H}^{\prime}\right)$. The maps in Proposition 5.1 induce natural maps

$$
\mathcal{Q}^{\prime} \hookrightarrow \mathcal{Q}, \quad \text { and } \quad \mathfrak{q}^{\prime} \hookrightarrow \mathfrak{q} .
$$

Actually, $\mathcal{Q}$ is isomorphic to the affine space $\mathbf{A}^{n}$, and the quotient map $\pi: \mathcal{S} \rightarrow$ $\mathcal{Q}$ is given by

$$
\left(\begin{array}{ll}
A & B \\
C & D
\end{array}\right) \mapsto\left(\operatorname{tr} \wedge^{i} B C\right), \quad i=1,2, \ldots, n
$$

The natural map $\mathcal{Q}^{\prime} \hookrightarrow \mathcal{Q}$ is induced by

$$
\mathcal{S}^{\prime} \longrightarrow \mathcal{Q}, \quad\left(\begin{array}{cc}
A & \gamma B \\
\bar{B} & \bar{A}
\end{array}\right) \mapsto\left(\operatorname{tr} \wedge^{i} \gamma B \bar{B}\right), \quad i=1, \ldots, n .
$$

Similarly, $\mathfrak{q}$ is isomorphic to the affine space $\mathbf{A}^{n}$, and the quotient map $\pi: \mathfrak{s} \rightarrow \mathfrak{q}$ is given by

$$
\left(\begin{array}{cc}
0 & A \\
B & 0
\end{array}\right) \mapsto\left(\operatorname{tr} \wedge^{i} A B\right), \quad i=1,2, \ldots, n
$$

The natural map $\mathfrak{q}^{\prime} \hookrightarrow \mathfrak{q}$ is induced by

$$
\mathfrak{s}^{\prime} \longrightarrow \mathfrak{q}, \quad\left(\begin{array}{cc}
0 & \gamma B \\
\bar{B} & \overline{0}
\end{array}\right) \mapsto\left(\operatorname{tr} \wedge^{i} \gamma B \bar{B}\right), \quad i=1, \ldots, n .
$$

Remark 5.4. A semisimple element $x=x\left(A, n_{1}, n_{2}\right)$ in $S_{\mathrm{ss}}$ comes from $S_{\mathrm{ss}}^{\prime}$ if and only if $A^{2}-\mathbf{1}_{m} \in \gamma \mathrm{N}\left(\mathrm{GL}_{m}(E)\right)$ where $m=n-n_{1}-n_{2}$. A semisimple element $X=X(A)$ in $\mathfrak{s}_{\mathrm{ss}}(F)$ comes from $\mathfrak{s}_{\mathrm{ss}}^{\prime}(F)$ if and only if $A \in \gamma \mathrm{N}\left(\mathrm{GL}_{m}(E)\right)$.

Remark 5.5. Suppose that $x \in S_{\mathrm{ss}}$ and $y \in S_{\mathrm{ss}}^{\prime}$ match. We want to compare $\left(H_{x}, \mathfrak{s}_{x}\right)$ with $\left(H_{y}^{\prime}, \mathfrak{s}_{y}^{\prime}\right)$. It suffices to assume that $x=x\left(A, n_{1}, n_{2}\right)$ and $y=$ $y\left(A, n_{1}, n_{2}\right)$. Thus, by Propositions 4.1 and 4.6 , we have

$$
\left(H_{x}, \mathfrak{s}_{x}\right) \simeq\left(\mathrm{GL}_{m}(F)_{A}, \mathfrak{g l}_{m}(F)_{A}\right) \times\left(H_{n_{1}}, \mathfrak{s}_{n_{1}}\right) \times\left(H_{n_{2}}, \mathfrak{s}_{n_{2}}\right),
$$

and

$\left(H_{y}^{\prime}, \mathfrak{s}_{y}^{\prime}\right) \simeq\left(\mathrm{GL}_{m}(E)_{A} \cap \mathrm{GL}_{\sigma, m}(E)_{B}, \mathfrak{g l}_{m}(E)_{A} \cap \mathfrak{g l}_{m}^{\sigma}(E)_{B}\right) \times\left(H_{n_{1}}^{\prime}, \mathfrak{s}_{n_{1}}^{\prime}\right) \times\left(H_{n_{2}}^{\prime}, \mathfrak{s}_{n_{2}}^{\prime}\right)$

with $A^{2}-\mathbf{1}_{m}=\gamma B \bar{B}$ and $A B=B A$. By the proof of Lemma 5.26 below, we see that $\left(\mathrm{GL}_{m}(E)_{A} \cap \mathrm{GL}_{\sigma, m}(E)_{B}, \mathfrak{g l}_{m}(E)_{A} \cap \mathfrak{g l}_{m}^{\sigma}(E)_{B}\right)$ essentially is an inner form of $\left(\mathrm{GL}_{m}(F)_{A}, \mathfrak{g l}_{m}(F)_{A}\right)$. The other factors in the descendants are related in a similar manner as $(H, \mathfrak{s})$ and $\left(H^{\prime}, \mathfrak{s}^{\prime}\right)$ are. For $X \in \mathfrak{s}_{\mathrm{SS}}(F)$ and $Y \in \mathfrak{s}_{\mathrm{sS}}^{\prime}(F)$ such that $X \leftrightarrow Y$, by Propositions 4.2 and 4.7 and Lemma 5.26, the factors of the descendants $\left(H_{X}, \mathfrak{s}_{X}\right)$ and $\left(H_{Y}^{\prime}, \mathfrak{s}_{Y}^{\prime}\right)$ have the similar relations as above.

Remark 5.6. It is obvious that the maps in Proposition 5.1 send regular semisimple orbits to regular semisimple ones. We denote by $S_{\mathrm{rs}, 0}\left(\operatorname{resp} . \mathfrak{s}_{\mathrm{rs}}(F)_{0}\right)$ the subset of elements in $S_{\mathrm{rs}}\left(\right.$ resp. $\left.\mathfrak{s}_{\mathrm{rs}}(F)\right)$ coming from $S_{\mathrm{rs}}^{\prime}\left(\operatorname{resp} . \mathfrak{s}_{\mathrm{rs}}^{\prime}(F)\right)$. Suppose that $x \in S_{\mathrm{rs}}\left(\right.$ resp. $\left.x \in \mathfrak{s}_{\mathrm{rs}}(F)\right)$ and $y \in S_{\mathrm{rs}}^{\prime}\left(\right.$ resp. $\left.y \in \mathfrak{s}_{\mathrm{rs}}^{\prime}(F)\right)$ match. Then by the above remark, we see that $H_{x}$ is an inner form of $H_{y}^{\prime}$. Since they are torus, we have

$$
H_{x} \simeq H_{y}^{\prime}
$$


Transfer factors To state our results on smooth transfer, we need to define transfer factors for the symmetric pair $(\mathbf{G}, \mathbf{H}, \theta)$ and its descendants. In general, the transfer factor is defined as follows (cf. [Zhw1, Definition 3.2]).

Definition 5.7. Let a reductive group $\mathbf{H}$ act on an affine variety $\mathbf{X}$, both defined over $F$. Let $\eta$ be a quadratic character of $H$. Suppose that for all regular semisimple $x \in X=\mathbf{X}(F)$, the character $\eta$ is trivial on the stabilizer $H_{x}$. Then a transfer factor is a smooth function $\kappa: X_{\mathrm{rs}} \rightarrow \mathbb{C}^{\times}$such that $\kappa\left(x^{h}\right)=\eta(h) \kappa(x)$ for any $h \in H$.

Definition 5.8. For convenience, we give an explicit definition of various transfer factors in our situation as follows:

- type $(H, S)$ : for $x=\left(\begin{array}{ll}A & B \\ C & D\end{array}\right) \in S$ regular semisimple, define $\kappa(x):=$ $\eta(\operatorname{det}(B))$

- type $\left(H_{m}, \mathfrak{s}_{m}\right)$ : for $X=\left(\begin{array}{cc}0 & A \\ B & 0\end{array}\right) \in \mathfrak{s}_{m}(F)$ regular semisimple, define $\kappa(X):=\eta(\operatorname{det}(A))$;

- type $\left(\mathrm{GL}_{m}(F)_{A}, \mathfrak{g l}_{m}(F)_{A}\right)$ : we define $\kappa$ to be the constant function with value 1 .

In the cases (1) and (2), $\eta$ is the non-trivial quadratic character on $F^{\times}$associated to $E$, while in the case (3) $\eta$ is the trivial character. In all the cases, it is easy to see that $\eta$ is trivial on the stabilizers $H_{x}$.

Smooth transfer Now we give the definition of smooth transfer. First, we fix Haar measures on $H$ and $H^{\prime}$. Notice that, for $x \in S_{\mathrm{rs}}\left(\operatorname{resp} . x \in \mathfrak{s}_{\mathrm{rs}}(F)\right)$ and $y \in S_{\mathrm{rs}}^{\prime}$ (resp. $\left.y \in \mathfrak{s}_{\mathrm{rs}}^{\prime}(F)\right)$ such that $x \leftrightarrow y$, their stabilizers $H_{x}$ and $H_{y}^{\prime}$ are isomorphic to each other (see Remark 5.6), and we fix such an isomorphism. Fix a Haar measure on $H_{x}$ for each $x \in S_{\text {rs }}$ (resp. $\left.x \in \mathfrak{s}_{\text {rs }}(F)\right)$. We fix a Haar measure on $H_{y}^{\prime}$ for each $y \in S_{\mathrm{rs}}^{\prime}$ (resp. $\left.y \in \mathfrak{s}_{\mathrm{rs}}^{\prime}(F)\right)$ which is compatible with that of $H_{x}$ if $x \leftrightarrow y$.

Definition 5.9. For $x \in S_{\mathrm{rs}}$ (resp. $\left.x \in \mathfrak{s}_{\mathrm{rs}}(F)\right)$ and $f \in \mathcal{C}_{c}^{\infty}(S)$ (resp. $f \in$ $\mathcal{C}_{c}^{\infty}(\mathfrak{s}(F))$ ), define the orbital integral of $f$ at $x$ to be

$$
O^{\eta}(x, f):=\int_{H_{x} \backslash H} f\left(x^{h}\right) \eta(h) \mathrm{d} h .
$$

For $y \in S_{\mathrm{rs}}^{\prime}\left(\operatorname{resp} . y \in \mathfrak{s}_{\mathrm{rs}}^{\prime}(F)\right)$ and $f^{\prime} \in \mathcal{C}_{c}^{\infty}\left(S^{\prime}\right)\left(\right.$ resp. $\left.f^{\prime} \in \mathcal{C}_{c}^{\infty}\left(\mathfrak{s}^{\prime}(F)\right)\right)$, define the orbital integral of $f^{\prime}$ at $y$ to be

$$
O(y, f):=\int_{H_{y}^{\prime} \backslash H^{\prime}} f\left(x^{h}\right) \mathrm{d} h .
$$

Definition 5.10. 1. For $f \in \mathcal{C}_{c}^{\infty}(S)$ and $f^{\prime} \in \mathcal{C}_{c}^{\infty}\left(S^{\prime}\right)$, we say that $f$ and $f^{\prime}$ are smooth transfer of each other if for each $x \in S_{\mathrm{rs}}$

$$
\kappa(x) O^{\eta}(x, f)=\left\{\begin{array}{cl}
O\left(y, f^{\prime}\right), & \text { if there exists } y \in S_{\mathrm{rs}}^{\prime} \text { such that } x \leftrightarrow y, \\
0, & \text { otherwise. }
\end{array}\right.
$$

We denote by $\mathcal{C}_{c}^{\infty}(S)_{0}$ the subspace of elements $f$ in $\mathcal{C}_{c}^{\infty}(S)$ satisfying that $O^{\eta}(x, f)=0$ for any $x$ in $S_{\mathrm{rs}}$ but not in $S_{\mathrm{rs}, 0}$. 
2. For $f \in \mathcal{C}_{c}^{\infty}(\mathfrak{s}(F))$ and $f^{\prime} \in \mathcal{C}_{c}^{\infty}\left(\mathfrak{s}^{\prime}(F)\right)$, we say that $f$ and $f^{\prime}$ are smooth transfer of each other if for each $X \in \mathfrak{s}_{\mathrm{rs}}(F)$

$$
\kappa(X) O^{\eta}(X, f)=\left\{\begin{array}{cl}
O\left(Y, f^{\prime}\right), & \text { if there exists } Y \in \mathfrak{s}_{\mathrm{rs}}^{\prime}(F) \text { such that } X \leftrightarrow Y, \\
0, & \text { otherwise. }
\end{array}\right.
$$

We denote by $\mathcal{C}_{c}^{\infty}(\mathfrak{s}(F))_{0}$ the subspace of elements $f$ in $\mathcal{C}_{c}^{\infty}(\mathfrak{s}(F))$ satisfying that $O^{\eta}(X, f)=0$ for any $X$ in $\mathfrak{s}_{\mathrm{rs}}(F)$ but not in $\mathfrak{s}_{\mathrm{rs}}(F)_{0}$.

Remark 5.11. The definition of smooth transfer depends on the Haar measures on $H$ and $H^{\prime}$, but the existence of smooth transfer does not depend on them. Sometimes, we will write transfer in place of smooth transfer for short.

Remark 5.12. For semisimple $x \in S$ and semisimple $y \in S^{\prime}$ such that $x \leftrightarrow y$, by Remark 5.5, we can define the notion of smooth transfer between elements in $\mathcal{C}_{c}^{\infty}\left(\mathfrak{s}_{x}(F)\right)$ and those in $\mathcal{C}_{c}^{\infty}\left(\mathfrak{s}_{y}^{\prime}(F)\right)$, determined by the orbital integrals with respect to the action of $H_{x}$ on $\mathfrak{s}_{x}(F)$, the action of $H_{y}^{\prime}$ on $\mathfrak{s}_{y}^{\prime}(F)$, and the transfer factor $\kappa$ defined as above. Similarly, for semisimple $X \in \mathfrak{s}(F)$ and semisimple $Y \in \mathfrak{s}^{\prime}(F)$ such that $X \leftrightarrow Y$, we can also define the notion of smooth transfer between elements in $\mathcal{C}_{c}^{\infty}\left(\mathfrak{s}_{X}(F)\right)$ and those in $\mathcal{C}_{c}^{\infty}\left(\mathfrak{s}_{Y}^{\prime}(F)\right)$.

Our main theorems are as follows.

Theorem 5.13. For each $f^{\prime} \in \mathcal{C}_{c}^{\infty}\left(S^{\prime}\right)$, there exists $f \in \mathcal{C}_{c}^{\infty}(S)$ that is a smooth transfer of $f^{\prime}$. Conversely, for each $f \in \mathcal{C}_{c}^{\infty}(S)_{0}$, there exists $f^{\prime} \in \mathcal{C}_{c}^{\infty}\left(S^{\prime}\right)$ that is a smooth transfer of $f$.

Theorem 5.14. For each $f^{\prime} \in \mathcal{C}_{c}^{\infty}\left(\mathfrak{s}^{\prime}(F)\right)$, there exists $f \in \mathcal{C}_{c}^{\infty}(\mathfrak{s}(F))$ that is a smooth transfer of $f^{\prime}$. Conversely, for each $f \in \mathcal{C}_{c}^{\infty}(\mathfrak{s}(F))_{0}$, there exists $f^{\prime} \in \mathcal{C}_{c}^{\infty}\left(\mathfrak{s}^{\prime}(F)\right)$ that is a smooth transfer of $f$.

In the later subsections, we will show that Theorem 5.14 implies Theorem 5.13 .

Lemma 5.15. To prove Theorem 5.14, it suffices to prove it for the case $\mathfrak{s}=\mathfrak{s}_{\epsilon}$ when $\epsilon=1$.

Proof. Let

$$
\mathfrak{s}^{\prime}(F)=\left\{Y(B)=\left(\begin{array}{cc}
0 & B \\
\bar{B} & 0
\end{array}\right): B \in \mathfrak{g l}_{n}(E)\right\} .
$$

Choose a representative $\gamma \in F^{\times}$of the nontrivial element in $F^{\times} / \mathrm{N} E^{\times}$. Let

$$
\mathfrak{s}_{\gamma}^{\prime}(F)=\left\{Y_{\gamma}(B)=\left(\begin{array}{cc}
0 & \gamma B \\
\bar{B} & 0
\end{array}\right): B \in \mathfrak{g l}_{n}(E)\right\} .
$$

Identify $H^{\prime}$ with $\mathrm{GL}_{n}(E)$. Then there is a natural $H^{\prime}$-equivariant isomorphism

$$
j: \mathfrak{s}^{\prime}(F) \stackrel{\sim}{\rightarrow} \mathfrak{s}_{\gamma}^{\prime}(F), Y(B) \mapsto Y_{\gamma}(B),
$$

which implies the lemma. 
Fourier transform Define the Fourier transform $f \mapsto \widehat{f}$ on $\mathcal{C}_{c}^{\infty}(\mathfrak{s}(F)$ ) (resp. $\left.\mathcal{C}_{c}^{\infty}\left(\mathfrak{s}^{\prime}(F)\right)\right)$ with respect to the fixed bilinear form $\langle$,$\rangle and the additive charac-$ ter $\psi$. The following theorem is the key point in proving the existence of smooth transfer.

Theorem 5.16. There exists a nonzero constant $c \in \mathbb{C}$ such that if $f \in$ $\mathcal{C}_{c}^{\infty}(\mathfrak{s}(F))$ and $f^{\prime} \in \mathcal{C}_{c}^{\infty}\left(\mathfrak{s}^{\prime}(F)\right)$ are smooth transfer of each other then $\widehat{f}$ and $c \widehat{f}^{\prime}$ are also smooth transfer of each other.

In the later subsections, we will prove the following main result of this section.

Proposition 5.17. Theorem 5.16 implies Theorem 5.14.

\subsection{Fundamental lemma}

In this subsection, we prove the following fundamental lemma (Lemma 5.18). This is an important example of the smooth transfer and also a crucial lemma for us to prove Theorem 5.16 by using global method.

Now assume that $\gamma=1$. Thus, $\mathbf{G}^{\prime}$ is isomorphic to $\mathbf{G}$. Suppose that $F$ is of odd residual characteristic and $E$ is unramified over $F$. We choose the Haar measures on $H$ and $H^{\prime}$ so that $\operatorname{vol}\left(\mathbf{H}\left(\mathcal{O}_{F}\right)\right)=1$ and $\operatorname{vol}\left(\mathbf{H}^{\prime}\left(\mathcal{O}_{F}\right)\right)=1$ respectively.

Let $f_{0} \in \mathcal{C}_{c}^{\infty}(\mathfrak{s}(F))$ and $f_{0}^{\prime} \in \mathcal{C}_{c}^{\infty}\left(\mathfrak{s}^{\prime}(F)\right)$ be the characteristic functions of the standard lattices

$$
L=\mathfrak{g l}_{n}\left(\mathcal{O}_{F}\right) \oplus \mathfrak{g l}_{n}\left(\mathcal{O}_{F}\right), \quad L^{\prime}=\mathfrak{g l}_{n}\left(\mathcal{O}_{E}\right)
$$

respectively.

Lemma 5.18. $f_{0}$ and $f_{0}^{\prime}$ are smooth transfer of each other.

Remark 5.19. The group version of the above fundamental lemma was proved in [Gu1] (cf. [Gu1, Theorem]).

Proof. Let $X \in \mathfrak{s}_{\mathrm{rs}}(F)$. It suffices to consider $X$ of the form $\left(\begin{array}{cc}0 & \mathbf{1}_{n} \\ A & 0\end{array}\right)$ with $A \in \mathrm{GL}_{n}(F)$ being regular semisimple. Then we have

$$
\begin{aligned}
\kappa(X) O^{\eta}\left(X, f_{0}\right) & =\int_{H_{X}(F) \backslash H(F)} f_{0}\left(h_{1}^{-1} h_{2}, h_{2}^{-1} A h_{1}\right) \eta\left(h_{1} h_{2}\right) \mathrm{d} h_{1} \mathrm{~d} h_{2} \\
& =\int_{\left(\mathrm{GL}_{n}(F)_{A} \backslash \mathrm{GL}_{n}(F)\right) \times \mathrm{GL}_{n}(F)} f_{0}\left(h_{2}, h_{2}^{-1} h_{1}^{-1} A h_{1}\right) \eta\left(h_{2}\right) \mathrm{d} h_{2} \mathrm{~d} h_{1} .
\end{aligned}
$$

Let $K=\mathrm{GL}_{n}\left(\mathcal{O}_{F}\right)$ and $K^{\prime}=\mathrm{GL}_{n}\left(\mathcal{O}_{E}\right)$. For $r=\left(r_{i, j}\right) \in \mathfrak{g l}_{n}(\bar{F})$, put $|r|=$ $\max _{i, j}\left|r_{i, j}\right|_{F}$. Then for $r, t \in \mathfrak{g l}_{n}(F)$, the value $f(r, t) \neq 0$ if and only if $|r| \leq$ $1,|t| \leq 1$. Let $\Phi_{A}$ be the characteristic function of the set of $(r, t) \in \mathrm{GL}_{n}(F) \times$ $\mathrm{GL}_{n}(F)$ satisfying $|r| \leq 1,|t| \leq 1$ and $|\operatorname{det}(r t)|_{F}=|\operatorname{det} A|_{F}$. Then $\Phi_{A}$ belongs to $\mathcal{C}_{c}^{\infty}\left(\mathrm{GL}_{n}(F) \times \mathrm{GL}_{n}(F)\right)$ and is bi- $K$-invariant both for the variables $r$ and $t$. Let $\Psi_{A}$ be the function on $\mathrm{GL}_{n}(F)$ defined by

$$
\Psi_{A}(g)=\int_{\mathrm{GL}_{n}(F)} \Phi_{A}\left(h, h^{-1} g\right) \eta(h) \mathrm{d} h .
$$


Then $\Psi_{A}$ belongs to $\mathcal{C}_{c}^{\infty}\left(\mathrm{GL}_{n}(F)\right)$, and is bi- $K$-invariant (that is, $\Psi_{A}$ is a Hecke function). We have

$$
\kappa(X) O^{\eta}\left(X, f_{0}\right)=\int_{\mathrm{GL}_{n}(F)_{A} \backslash \mathrm{GL}_{n}(F)} \Psi_{A}\left(g^{-1} A g\right) \mathrm{d} g .
$$

If $Y=\left(\begin{array}{cc}0 & B \\ \bar{B} & 0\end{array}\right) \in \mathfrak{s}_{\mathrm{rs}}^{\prime}(F)$, we have

$$
O\left(Y, f_{0}^{\prime}\right)=\int_{\mathrm{GL}_{\sigma, n}(E)_{B} \backslash \mathrm{GL}_{n}(E)} f_{0}^{\prime}\left(h^{-1} B \bar{h}\right) \mathrm{d} h .
$$

Let $\Psi_{B}$ be the characteristic function of the set of $r \in \mathrm{GL}_{n}(E)$ satisfying $|r| \leq$ 1 and $|\operatorname{det} r|_{F}=|\operatorname{det} B|_{F}$. Then $\Psi_{B}$ belongs to $\mathcal{C}_{c}^{\infty}\left(\mathrm{GL}_{n}(E)\right)$, and is bi- $K^{\prime}-$ invariant. We have

$$
O\left(Y, f_{0}^{\prime}\right)=\int_{\mathrm{GL}_{\sigma, n}(E)_{B} \backslash \mathrm{GL}_{n}(E)} \Psi_{B}\left(h^{-1} B \bar{h}\right) \mathrm{d} h .
$$

Denote by

$$
\text { bc : } \mathcal{H}\left(\mathrm{GL}_{n}(E), K^{\prime}\right) \longrightarrow \mathcal{H}\left(\mathrm{GL}_{n}(F), K\right)
$$

the base change map between the two spaces of Hecke functions. Then, in fact, it was shown in [Gu1, Corollary 3.7] (can be read off from the proof of Proposition 3.7 loc. cit.) that $\Psi_{A}=0$ if $A \notin \mathrm{N}\left(\mathrm{GL}_{n}(E)\right)$, and $\Psi_{A}=\mathrm{bc}\left(\Psi_{B}\right)$ if $A=B \bar{B}$. Recall that $\Psi_{A}=\mathrm{bc}\left(\Psi_{B}\right)$ implies that

$$
\kappa(X) O^{\eta}\left(X, f_{0}\right)=O\left(Y, f_{0}^{\prime}\right), \quad \text { if } X \leftrightarrow Y .
$$

Hence the lemma follows.

\subsection{Reduction steps}

The main aim of this subsection is to reduce Theorem 5.13 to Theorem 5.14. The reduction steps here are almost the same as those in [Zhw1, Section 3].

Descent of orbital integrals The following proposition essentially is [Zhw1, Proposition 3.11], whose proof is also valid here.

Proposition 5.20. Let $X$ be any one of $S, S^{\prime}, \mathfrak{s}(F)$ or $\mathfrak{s}^{\prime}(F)$. Let $x \in X$ be semisimple and $\left(U_{x}, p, \psi, Z_{x}, N_{x}\right)$ an analytic Luna slice at $x$. Then there exists a neighborhood $\xi \subset \psi\left(p^{-1}(x)\right)$ of 0 in $N_{x}$ satisfying the following properties:

- for each $f \in \mathcal{C}_{c}^{\infty}(X)$, there exists $f_{x} \in \mathcal{C}_{c}^{\infty}\left(N_{x}\right)$ such that for all regular semisimple $z \in \xi$ with $z=\psi(y)$ we have

$$
\int_{H_{y} \backslash H} f\left(y^{h}\right) \eta(h) \mathrm{d} h=\int_{H_{y} \backslash H_{x}} f_{x}\left(z^{h}\right) \eta(h) \mathrm{d} h ;
$$

- and conversely, for each $f_{x} \in \mathcal{C}_{c}^{\infty}\left(N_{x}\right)$, there exists $f$ in $\mathcal{C}_{c}^{\infty}(X)$ such that above equality holds for any regular semisimple $z \in \xi$.

Here $H=H^{\prime}$ and $\eta=\mathbf{1}$ when $X$ is $S^{\prime}$ or $\mathfrak{s}^{\prime}(F)$. 
Reduction to local transfer Recall that we denote by $\mathcal{Q}$ (resp. $\mathcal{Q}^{\prime}$ ) the categorical quotient $\mathcal{S} / \mathbf{H}$ (resp. $\mathcal{S}^{\prime} / \mathbf{H}^{\prime}$ ), and by $\mathfrak{q}$ (resp. $\mathfrak{q}^{\prime}$ ) the categorical quotient $\mathfrak{s} / \mathbf{H}\left(\right.$ resp. $\left.\mathfrak{s}^{\prime} / \mathbf{H}^{\prime}\right)$. By Remark 5.3, we always view $\mathcal{Q}^{\prime}$ and $\mathfrak{q}^{\prime}$ as closed subsets of $\mathcal{Q}$ and $\mathfrak{q}$ respectively. Let $\mathbf{X}$ be any one of $\mathcal{S}, \mathcal{S}^{\prime}, \mathfrak{s}$ or $\mathfrak{s}^{\prime}$, and $\mathbf{Q}$ the quotient $\mathcal{Q}, \mathcal{Q}^{\prime}, \mathfrak{q}$ or $\mathfrak{q}^{\prime}$ of $\mathbf{X}$. Let $\mathbf{Q}(F)_{\text {rs }}$ be the regular semisimple locus in $\mathbf{Q}(F)$. Since $H^{1}(F, \mathbf{H})=H^{1}\left(F, \mathbf{H}^{\prime}\right)=1$, the natural map $\pi: \mathbf{X}(F) \rightarrow \mathbf{Q}(F)$ is a surjection. For $x \in \mathbf{Q}(F)_{\mathrm{rs}}$, the fiber $\pi^{-1}(x)$ consists of precisely one orbit.

Definition 5.21. Let $\mathbf{X}$ and $\mathbf{Q}$ be as above. Write $X=\mathbf{X}(F)$ and $Q=\mathbf{Q}(F)$.

1. Let $\Phi$ be a function on $Q_{\mathrm{rs}}$ which vanishes outside a compact set of $Q_{\mathrm{rs}}$. For $x \in Q$, we say that $\Phi$ is a local orbital integral around $x$, if there exists a neighborhood $U$ of $x$ and a function $f \in \mathcal{C}_{c}^{\infty}(X)$ such that for all $y \in U_{\mathrm{rs}}$ and $z$ with $\pi(z)=y$ we have

$$
\Phi(y)=\kappa(z) O^{\eta}(z, f) .
$$

2. For $f \in \mathcal{C}_{c}^{\infty}(X)$, define a function $\pi_{*}(f)$ on $Q_{\mathrm{rs}}$ to be:

$$
\pi_{*}(f)(x)=\kappa(y) O^{\eta}(y, f), \quad \text { for } x \in Q_{\mathrm{rs}}, y \in \pi^{-1}(x) .
$$

Here $\kappa=\mathbf{1}$ and $\eta=\mathbf{1}$ when $\mathbf{X}$ is $\mathcal{S}^{\prime}$ or $\mathfrak{s}^{\prime}$.

The following result is [Zhw1, Proposition 3.8].

Proposition 5.22. Let $\Phi$ be a function on $Q_{\mathrm{rs}}$ which vanishes outside a compact set $\Xi$ of $Q$. If $\Phi$ is a local orbital integral at each $x \in \Xi$, it is an orbital integral. Namely there exists $f \in \mathcal{C}_{c}^{\infty}(X)$ such that for all $y \in Q_{\mathrm{rs}}$, and $z$ with $\pi(z)=y$ we have

$$
\Phi(y)=\kappa(z) O^{\eta}(z, f) .
$$

Definition 5.23. For $x \in \mathcal{Q}(F)$ (resp. $x \in \mathfrak{q}(F)$ ), we say that local transfer around $x$ exists, if for each $f^{\prime} \in \mathcal{C}_{c}^{\infty}\left(S^{\prime}\right)$ (resp. $f^{\prime} \in \mathcal{C}_{c}^{\infty}\left(\mathfrak{s}^{\prime}(F)\right)$ ), there exists $f \in \mathcal{C}_{c}^{\infty}(S)_{0}$ (resp. $\left.f \in \mathcal{C}_{c}^{\infty}(\mathfrak{s}(F))_{0}\right)$ such that in a neighborhood $U$ of $x$, the following equality holds:

$$
\pi_{*}(f)=\pi_{*}\left(f^{\prime}\right) \text { on } U \cap \mathcal{Q}(F)_{\mathrm{rs}}\left(\text { resp. } U \cap \mathfrak{q}(F)_{\mathrm{rs}}\right)
$$

and conversely for each $f \in \mathcal{C}_{c}^{\infty}(S)_{0}$ (resp. $\left.f \in \mathcal{C}_{c}^{\infty}(\mathfrak{s}(F))_{0}\right)$, there exists $f^{\prime} \in$ $\mathcal{C}_{c}^{\infty}\left(S^{\prime}\right)$ (resp. $f^{\prime} \in \mathcal{C}_{c}^{\infty}\left(\mathfrak{s}^{\prime}(F)\right)$ ) satisfying the above equality.

Corollary 5.24. To prove Theorems 5.13 and 5.14, it suffices to prove the existence of local transfer around all elements of $\mathcal{Q}(F)$ and $\mathfrak{q}(F)$.

Proof. This is a direct consequence of Proposition 5.22.

\section{Reduction to local transfer around zero}

Lemma 5.25. To prove the existence of local transfer around an element $z$ in $\mathcal{Q}(F)$ (resp. $\mathfrak{q}(F)$ ), it suffices to prove the existence of smooth transfer for the sliced representations $\left(H_{x}, \mathfrak{s}_{x}\right)$ and $\left(H_{y}^{\prime}, \mathfrak{s}_{y}^{\prime}\right)$ where $x$ in $S_{\mathrm{ss}}\left(\right.$ resp. $\left.\mathfrak{s}_{\mathrm{ss}}(F)\right)$ and $y$ in $S_{\mathrm{SS}}^{\prime}\left(\right.$ resp. $\left.\mathfrak{s}_{\mathrm{SS}}^{\prime}(F)\right)$ are such that $x \leftrightarrow y$ and $\pi(x)=\pi(y)=z$. 
Proof. This result partially follows from Proposition 5.20 and the fact that for $f^{\prime} \in \mathcal{C}_{c}^{\infty}\left(S^{\prime}\right)\left(\operatorname{resp} . \mathcal{C}_{c}^{\infty}\left(\mathfrak{s}^{\prime}(F)\right)\right)$ and $f \in \mathcal{C}_{c}^{\infty}(S)$ (resp. $\left.\mathcal{C}_{c}^{\infty}(\mathfrak{s}(F))\right)$ the functions $\pi_{*}\left(f^{\prime}\right)$ and $\pi_{*}(f)$ are locally constant on $\mathcal{Q}(F)_{\mathrm{rs}}$ (resp. $\left.\mathfrak{q}(F)_{\mathrm{rs}}\right)$. It remains to prove Lemma 5.29 ahead, which shows the compatibility of the transfer factors under the semisimple descent.

Lemma 5.26. 1. Given semisimple $A \in \mathfrak{g l}_{m}(F)$ such that $A^{2}-\mathbf{1}_{m}=\gamma B \bar{B}$, $A B=B A$ with $B \in \mathrm{GL}_{m}(E)$, the smooth transfer exists for the sliced representations

$\left(\mathrm{GL}_{m}(F)_{A}, \mathfrak{g l}_{m}(F)_{A}\right)$ and $\left(\mathrm{GL}_{m}(E)_{A} \cap \mathrm{GL}_{\sigma, m}(E)_{B}, \mathfrak{g l}_{m}(E)_{A} \cap \mathfrak{g l}_{m}^{\sigma}(E)_{B}\right)$.

2. Given semisimple $A \in \mathrm{GL}_{m}(F)$ such that $A=\gamma B \bar{B}$ with $B \in \mathrm{GL}_{m}(E)$, the smooth transfer exists for the sliced representations

$$
\left(\mathrm{GL}_{m}(F)_{A}, \mathfrak{g l}_{m}(F)_{A}\right) \text { and }\left(\mathrm{GL}_{\sigma, m}(E)_{B}, \mathfrak{g l}_{m}^{\sigma}(E)_{B}\right)
$$

Proof. Firstly, we prove the second assertion. We can assume that $\gamma=1$ and $A$ is of the form $\operatorname{diag}\left(A_{1}, A_{2}, \ldots, A_{k}\right)$ such that

$$
\mathrm{GL}_{m}(F)_{A}=\prod_{i=1}^{k} \mathrm{GL}_{m_{i}}\left(F_{i}\right),
$$

where $F_{i}=F\left[A_{i}\right]$ is a field and $A_{i}$ is in the center of $\mathrm{GL}_{m_{i}}\left(F_{i}\right)$. For each $1 \leq i \leq$ $k$, let $L_{i}=E \otimes_{F} F_{i}$. Since $A \in \mathrm{N}\left(\mathrm{GL}_{m}(E)\right)$, there exists $B_{i} \in \mathrm{GL}_{m_{i}}\left(L_{i}\right)$ such that $A_{i}=\mathrm{N}\left(B_{i}\right)$ for each $i$. We can choose $B$ to be $\operatorname{diag}\left(B_{1}, B_{2}, \ldots, B_{k}\right)$. Then $\operatorname{GL}_{\sigma, m_{i}}\left(L_{i}\right)_{B_{i}}$ is an inner form of $\mathrm{GL}_{m_{i}}\left(F_{i}\right)$, and $\mathrm{GL}_{\sigma, m}(E)_{B}=\prod_{i=1}^{k} \operatorname{GL}_{\sigma, m_{i}}\left(L_{i}\right)_{B_{i}}$. For $X \in \mathfrak{g l}_{m}^{\sigma}(E)_{B}$, it is easy to see that $X \bar{B} \in \mathfrak{g l}_{\sigma, m}(E)_{B}$, where

$$
\mathfrak{g l}_{\sigma, m}(E)_{B}=\left\{Y \in \mathfrak{g l}_{m}(E): Y B=B \bar{Y}\right\},
$$

which is the Lie algebra of $\mathrm{GL}_{\sigma, m}(E)_{B}$. For $X \in \mathfrak{g l}_{m}^{\sigma}(E)_{B}$ and $h \in \mathrm{GL}_{\sigma, m}(E)_{B}$, we have $h^{-1} X \bar{h} \bar{B}=h^{-1} X \bar{B} h$. Therefore, timing $\bar{B}$ on right, we get a $\mathrm{GL}_{\sigma, m}(E)_{B^{-}}$ equivariant isomorphism

$$
\mathfrak{g l}_{m}^{\sigma}(E)_{B} \longrightarrow \mathfrak{g l}_{\sigma, m}(E)_{B}
$$

where $\mathrm{GL}_{\sigma, m}(E)_{B}$ acts on $\mathfrak{g l}_{\sigma, m}(E)_{B}$ by conjugation. Since the existence of smooth transfer between $\mathrm{GL}_{m}\left(F_{i}\right)$ and its inner forms is known, we completes the proof.

The first assertion is proved in the same way. By the above discussion, we know that the smooth transfer holds for

$$
\left(\mathrm{GL}_{m}(F)_{A^{2}-\mathbf{1}_{m}}, \mathfrak{g l}_{m}(F)_{A^{2}-\mathbf{1}_{m}}\right) \text { and }\left(\mathrm{GL}_{\sigma, m}(E)_{B}, \mathfrak{g l}_{m}^{\sigma}(E)_{B}\right) \text {. }
$$

We can choose some scalar $\lambda \in F$ so that $A+\lambda \in \mathrm{GL}_{m}(F)$. Then $A+\lambda \in$ $\mathrm{GL}_{m}(F)_{A^{2}-\mathbf{1}_{m}}$ and $A+\lambda \in \mathrm{GL}_{\sigma, m}(E)_{B}$. Hence

$$
\left(\mathrm{GL}_{\sigma, m}(E)_{B}\right)_{A+\lambda}=\mathrm{GL}_{m}(E)_{A} \cap \mathrm{GL}_{\sigma, m}(E)_{B}
$$

is an inner form of

$$
\left(\mathrm{GL}_{m}(F)_{A^{2}-\mathbf{1}_{m}}\right)_{A+\lambda}=\mathrm{GL}_{m}(F)_{A} .
$$

The rest of the proof is the same as that of the first assertion. 
Proposition 5.27. To prove the existence of local transfer around all elements of $\mathcal{Q}(F)$ or $\mathfrak{q}(F)$, it suffices to prove the existence of local transfer around zero of $\mathfrak{q}(F)$.

Proof. By Lemma 5.25, it suffices to prove the existence of smooth transfer for the sliced representations $\left(H_{x}, \mathfrak{s}_{x}\right)$ and $\left(H_{y}^{\prime}, \mathfrak{s}_{y}^{\prime}\right)$ where $x \leftrightarrow y$. By Remark 5.5 and Lemma 5.26, it suffices to prove the existence of smooth transfer for $\left(H_{m}, \mathfrak{s}_{m}\right)$ and $\left(H_{m}^{\prime}, \mathfrak{s}_{m}^{\prime}\right)$, that is, the existence of local transfer around zero of $\mathfrak{q}(F)$.

Corollary 5.28. Theorem 5.14 implies Theorem 5.13.

Explicit analytic Luna slices We now describe explicit analytic Luna slices at semisimple elements of $S$ or $\mathfrak{s}(F)$. We refer the reader to [JR, page 76] for the discussions on $\mathfrak{s}$, and to [JR, $\S 5.2]$ for the discussions on $S$.

First let $X \in \mathfrak{s}(F)$ be semisimple. Write $\mathfrak{s}(F)=\mathfrak{s}_{X} \oplus \mathfrak{s}_{X}^{\perp}$, where $\mathfrak{s}_{X}^{\frac{1}{X}}$ is the orthogonal complement of $\mathfrak{s}_{X}$ in $\mathfrak{s}(F)$ with respect to $\langle$,$\rangle . Set$

$$
Z=\left\{\xi \in \mathfrak{s}_{X}: \operatorname{det}\left(\left.\left[\operatorname{ad}(X+\xi)^{2}\right]\right|_{\mathfrak{s}_{X}^{\frac{1}{X}}}\right) \neq 0\right\},
$$

which is a non-empty open set of $\mathfrak{s}_{X}$ and invariant under $H_{X}$. Let $Z_{X}=\{X+\xi$ : $\xi \in Z\}$. Consider the map

$$
\phi: H \times Z_{X} \longrightarrow \mathfrak{s}(F), \quad(h, X+\xi) \mapsto \operatorname{Ad} h(X+\xi),
$$

which is everywhere submersive. Let $U_{X}$ be the image of $\phi$, which is an open $H$-invariant set in $\mathfrak{s}(F)$. Then $Z_{X}$ and $U_{X}$ are what we want, and $\psi$ is the natural map:

$$
\psi: Z_{X} \longrightarrow \mathfrak{s}_{X}, \quad X+\xi \mapsto \xi .
$$

Next let $x \in S$ be semisimple. Write $x=s(g)$ for some $g \in G$, where $s$ is the symmetrization map. Consider the map

$$
\phi: H \times G_{x} \times H \longrightarrow G, \quad\left(h, \xi, h^{\prime}\right) \mapsto h \xi g h^{\prime} .
$$

Let $\mathcal{Z}^{\prime}$ be the set of $\xi$ such that $\phi$ is submersive at $(1, \xi, 1)$, which is also the set of $\xi$ in $G_{x}$ such that

$$
\operatorname{det}\left(\left.[\mathbf{1}-\operatorname{Ad} s(\xi g)]\right|_{\mathfrak{g}_{x}^{\perp}}\right) \neq 0 .
$$

Let

$$
W^{\prime}=\left\{X \in \mathfrak{s}_{x}: \operatorname{det}(\mathbf{1}+X) \operatorname{det}(\mathbf{1}-X) \neq 0\right\},
$$

which is an open neighborhood of 0 in $\mathfrak{s}_{x}$. Consider the Cayley transform

$$
\lambda: W^{\prime} \longrightarrow G_{x}, \quad X \mapsto(\mathbf{1}-X)(\mathbf{1}+X)^{-1},
$$

and denote by $\mathcal{V}$ the image of $W^{\prime}$ under $\lambda$. Put $\mathcal{Z}=\mathcal{Z}^{\prime} \cap \mathcal{V}$ and $W=\lambda^{-1}(\mathcal{Z})$. Let $U_{x}$ be the image of $\phi(H \times \mathcal{Z} \times H)$ under the symmetrization map $s$, and $Z_{x}$ the image of $\phi(\mathbf{1} \times \mathcal{Z} \times \mathbf{1})$ under $s$. Then $Z_{x}$ and $U_{x}$ are what we want.

The lemma below follows from the above construction and a direct computation by choosing $x=x\left(A, n_{1}, n_{2}\right)$ and $X=X(A)$ in a standard form. We omit the proof here.

Lemma 5.29. Let $x \in S$ (resp. $X \in \mathfrak{s}(F)$ ) be semisimple. Then we may choose an $H_{x}$-invariant (resp. $H_{X}$-invariant) neighborhood of $x$ (resp. $\left.X\right)$ such that for any regular semisimple $y$ in this neighborhood, $\kappa(y)$ is equal to a non-zero constant times $\kappa(\psi(y))$. 


\subsection{Proof of Proposition 5.17}

Now we can prove Proposition 5.17 with the help of the following results.

Theorem 5.30. Denote by $\mathcal{N}$ the null-cone of $\mathfrak{s}(F)$, by $\mathcal{N}^{\prime}$ the null-cone of $\mathfrak{s}^{\prime}(F)$.

1. Let $T \in \mathcal{D}(\mathfrak{s}(F))^{H, \eta}$ be such that $\operatorname{Supp}(T) \subset \mathcal{N}$ and $\operatorname{Supp}(\widehat{T}) \subset \mathcal{N}$. Then $T=0$.

2. Let $T \in \mathcal{D}\left(\mathfrak{s}^{\prime}(F)\right)^{H^{\prime}}$ be such that $\operatorname{Supp}(T) \subset \mathcal{N}^{\prime}$ and $\operatorname{Supp}(\widehat{T}) \subset \mathcal{N}^{\prime}$. Then $T=0$.

Proof. The first assertion is proved in [JR, Proposition 3.1] when $\eta$ is the trivial character. The same proof goes through for the quadratic character $\eta$. The same proof is also valid for the second assertion, noting the relation $m^{\prime}<n^{2}$ in Proposition 4.8.

The following corollary is a direct consequence of the above theorem (cf. [Zhw1, Corollary 4.20]).

Corollary 5.31. 1. Let $\mathcal{C}_{0}=\bigcap_{T} \operatorname{ker}(T)$ where $T$ runs over all $(H, \eta)$-invariant distributions on $\mathfrak{s}(F)$. Then each $f \in \mathcal{C}_{c}^{\infty}(\mathfrak{s}(F))$ can be written as

$$
f=f_{0}+f_{1}+\widehat{f_{2}}
$$

with $f_{0} \in \mathcal{C}_{0}$ and $f_{i} \in \mathcal{C}_{c}^{\infty}(\mathfrak{s}(F)-\mathcal{N}), i=1,2$.

2. Let $\mathcal{C}_{0}=\bigcap_{T} \operatorname{ker}(T)$ where $T$ runs over all $H^{\prime}$-invariant distributions on $\mathfrak{s}^{\prime}(F)$. Then each $f \in \mathcal{C}_{c}^{\infty}\left(\mathfrak{s}^{\prime}(F)\right)$ can be written as

$$
f=f_{0}+f_{1}+\widehat{f_{2}}
$$

with $f_{0} \in \mathcal{C}_{0}$ and $f_{i} \in \mathcal{C}_{c}^{\infty}\left(\mathfrak{s}^{\prime}(F)-\mathcal{N}^{\prime}\right), i=1,2$.

Proof of Proposition 5.17. Now we assume that Theorem 5.16 is true. First we consider the converse direction: given $f \in \mathcal{C}_{c}^{\infty}(\mathfrak{s}(F))_{0}$, we want to show its smooth transfer exists in $\mathcal{C}_{c}^{\infty}\left(\mathfrak{s}^{\prime}(F)\right)$. For a general element $f$ in $\mathcal{C}_{c}^{\infty}(\mathfrak{s}(F))$, we say that $f^{\prime} \in \mathcal{C}_{c}^{\infty}\left(\mathfrak{s}^{\prime}(F)\right)$ is a smooth transfer of $f$ if

$$
O\left(y, f^{\prime}\right)=\kappa(x) O^{\eta}(x, f), \quad x \leftrightarrow y,
$$

for each $y \in \mathfrak{s}_{\text {rs }}^{\prime}(F)$. We can and do assume that: there exists a nonzero $c \in \mathbb{C}$ such that if $f^{\prime}$ is a smooth transfer of $f \in \mathcal{C}_{c}^{\infty}(\mathfrak{s}(F))$ then $c \widehat{f}^{\prime}$ is a smooth transfer of $\widehat{f}$. This assumption is proved in Theorem 8.2. Basing on this assumption, we will show the following stronger form of Theorem 5.14: for each $f \in \mathcal{C}_{c}^{\infty}(\mathfrak{s}(F)$ ), there exists $f^{\prime} \in \mathcal{C}_{c}^{\infty}\left(\mathfrak{s}^{\prime}(F)\right)$ that is a smooth transfer of $f$. We use induction argument to show this result. Suppose that the stronger form of Theorem 5.14 holds for $\mathcal{C}_{c}^{\infty}\left(\mathfrak{s}_{m}(F)\right)$ and $\mathcal{C}_{c}^{\infty}\left(\mathfrak{s}_{m}^{\prime}(F)\right)$ for every $m<n$. Thus, by Corollary 5.24 and Lemma 5.26, for each $f \in \mathcal{C}_{c}^{\infty}(\mathfrak{s}(F)-\mathcal{N})$, its smooth transfer exists. Therefore, by Corollary 5.31, it suffices to show the existence of smooth transfer for $\widehat{f}$ with $f \in \mathcal{C}_{c}^{\infty}(\mathfrak{s}(F)-\mathcal{N})$, which is guaranteed by the assumption.

For the other direction in Theorem 5.14 the proof is the same. 


\section{Representability}

For $X \in \mathfrak{s}_{\mathrm{rs}}(F)$, it is more convenient to consider the normalized orbital integral

$$
I^{\eta}(X, f):=\left|D^{\mathfrak{s}}(X)\right|_{F}^{\frac{1}{2}} O^{\eta}(X, f), \quad f \in \mathcal{C}_{c}^{\infty}(\mathfrak{s}(F)) .
$$

Similarly, for $Y \in \mathfrak{s}_{\mathrm{rs}}^{\prime}(F)$, we consider the normalized orbital integral

$$
I\left(Y, f^{\prime}\right):=\left|D^{\mathfrak{s}^{\prime}}(Y)\right|_{F}^{\frac{1}{2}} O\left(Y, f^{\prime}\right), \quad f^{\prime} \in \mathcal{C}_{c}^{\infty}\left(\mathfrak{s}^{\prime}(F)\right) .
$$

If $X \leftrightarrow Y$ it is not hard to see that $\left|D^{\mathfrak{s}}(X)\right|_{F}=\left|D^{\mathfrak{s}^{\prime}}(Y)\right|_{F}$. Hence it does not matter if we consider the smooth transfer with respect to the normalized orbital integrals instead of the orbital integrals introduced before. The Fourier transform of the normalized orbital integral $I_{X}^{\eta}$ is defined to be

$$
\widehat{I}^{\eta}(X, f)=I^{\eta}(X, \widehat{f}) .
$$

For $Y \in \mathfrak{s}_{\text {rs }}^{\prime}(F)$, we define $\widehat{I}_{Y}$ similarly.

To prove Theorem 5.16, we first need to study the Fourier transform of orbital integrals. In this section, we prove the following fundamental theorem on the representability of $\widehat{I}_{X}^{\eta}$ and $\widehat{I}_{Y}$.

Theorem 6.1. 1. For each $X \in \mathfrak{s}_{\mathrm{rs}}(F)$, there exists a locally constant $H$ invariant function $\widehat{i}_{X}^{\eta}$ defined on $\mathfrak{s}_{\mathrm{rs}}(F)$ which is locally integrable on $\mathfrak{s}(F)$, such that for any $f \in \mathcal{C}_{c}^{\infty}(\mathfrak{s}(F))$ we have

$$
\widehat{I}^{\eta}(X, f)=\int_{\mathfrak{s}(F)} \widehat{i}_{X}^{\eta}(Y) \kappa(Y) f(Y)\left|D^{\mathfrak{s}}(Y)\right|_{F}^{-1 / 2} \mathrm{~d} Y .
$$

2. For each $X \in \mathfrak{s}_{\mathrm{rs}}^{\prime}(F)$, there exits a locally constant $H^{\prime}$-invariant function $\widehat{i}_{X}$ defined on $\mathfrak{s}_{\mathrm{rs}}^{\prime}(F)$ which is locally integrable on $\mathfrak{s}^{\prime}(F)$, such that for any $f \in \mathcal{C}_{c}^{\infty}\left(\mathfrak{s}^{\prime}(F)\right)$ we have

$$
\widehat{I}(X, f)=\int_{\mathfrak{s}^{\prime}(F)} \widehat{i}_{X}(Y) f(Y)\left|D^{\mathfrak{s}^{\prime}}(Y)\right|_{F}^{-1 / 2} \mathrm{~d} Y .
$$

We also write $\widehat{i}^{\eta}(X, Y)$ (resp. $\left.\widehat{i}(X, Y)\right)$ instead of $\widehat{i}_{X}^{\eta}(Y)$ (resp. $\widehat{i}_{X}(Y)$ ), which is viewed as a function on $\mathfrak{s}_{\mathrm{rs}}(F) \times \mathfrak{s}_{\mathrm{rs}}(F)\left(\right.$ resp. $\left.\mathfrak{s}_{\mathrm{rs}}^{\prime}(F) \times \mathfrak{s}_{\mathrm{rs}}^{\prime}(F)\right)$. Then it is not hard to see that $\widehat{i}^{\eta}(X, Y)$ (resp. $\widehat{i}(X, Y)$ ) is locally constant on $\mathfrak{s}_{\mathrm{rs}}(F) \times \mathfrak{s}_{\mathrm{rs}}(F)$ (resp. $\left.\mathfrak{s}_{\mathrm{rs}}^{\prime}(F) \times \mathfrak{s}_{\mathrm{rs}}^{\prime}(F)\right),(H, \eta)$-invariant (resp. $H^{\prime}$-invariant) on the first variable and $H$-invariant (resp. $H^{\prime}$-invariant) on the second variable. Our method to prove Theorem 6.1 follows that of of [HC1] and [HC2]. Some of our treatment also follows that of $[\mathrm{Ko}]$. We only prove the assertion for $\widehat{I}_{X}^{\eta}$. The assertion for $\widehat{I}_{X}$ can be proved in the same way and is left to the reader.

\subsection{Reduction to elliptic case}

In this subsection we reduce the question of the representability of $\widehat{I}_{X}^{\eta}$ to that for elliptic elements $X \in \mathfrak{s}_{\mathrm{rs}}(F)$. For $X \in \mathfrak{s}_{\mathrm{rs}}(F)$, we say that $X$ is elliptic if its stabilizer $H_{X}$ is an elliptic torus. Thus, if $X=\left(\begin{array}{cc}0 & A \\ B & 0\end{array}\right), X$ is elliptic if and only $A B$ is elliptic in $\mathrm{GL}_{n}(F)$ in the usual sense. 
For convenience, we suppose that $X \in \mathfrak{s}_{\mathrm{rs}}(F)$ is of the form $\left(\begin{array}{cc}0 & \mathbf{1}_{n} \\ A & 0\end{array}\right)$. From now on, we also suppose that $X$ is not elliptic, or equivalently, $A$ is not elliptic. Then there exists a proper Levi subgroup $\mathbf{M}_{0}$ of $\mathrm{GL}_{n}$ such that $A \in \mathbf{M}_{0}$. Let $\mathbf{P}_{0}$ be a proper parabolic subgroup of $\mathrm{GL}_{n}$ such that $\mathbf{M}_{0}$ is a Levi component of $\mathbf{P}_{0}$. Let $\mathbf{U}_{0}$ be the unipotent subgroup of $\mathbf{P}_{0}$. Set $\mathfrak{m}_{0}=\operatorname{Lie}\left(\mathbf{M}_{0}\right), \mathfrak{p}_{0}=\operatorname{Lie}\left(\mathbf{P}_{0}\right)$ and $\mathfrak{u}_{0}=\operatorname{Lie}\left(\mathbf{U}_{0}\right)$. Then $\mathfrak{p}_{0}=\mathfrak{m}_{0} \oplus \mathfrak{u}_{0}$, and $\mathfrak{g l}_{n}=\mathfrak{p}_{0} \oplus \overline{\mathfrak{u}}_{0}$ where $\overline{\mathfrak{u}}_{0}$ is the Lie algebra of the unipotent subgroup $\overline{\mathbf{U}}_{0}$ opposite to $\mathbf{U}_{0}$.

Write $\mathfrak{s}=\mathfrak{s}^{+} \oplus \mathfrak{s}^{-}$, where

$$
\mathfrak{s}^{+}=\left\{\left(\begin{array}{cc}
0 & B \\
0 & 0
\end{array}\right): B \in \mathfrak{g l}_{n}\right\}, \quad \mathfrak{s}^{-}=\left\{\left(\begin{array}{cc}
0 & 0 \\
C & 0
\end{array}\right): C \in \mathfrak{g l}_{n}\right\} .
$$

Identify $\mathfrak{s}^{+}\left(\right.$resp. $\left.\mathfrak{s}^{-}\right)$with $\mathfrak{g l}_{n}$. Under this identification, let $\mathfrak{r}^{+} \subset \mathfrak{s}^{+}$(resp. $\left.\mathfrak{r}^{-} \subset \mathfrak{s}^{-}\right)$be the subspace that corresponds to $\mathfrak{m}_{0}, \mathfrak{n}^{+} \subset \mathfrak{s}^{+}\left(\right.$resp. $\left.\mathfrak{n}^{-} \subset \mathfrak{s}^{-}\right)$ the subspace that corresponds to $\mathfrak{u}_{0}, \overline{\mathfrak{n}}^{+} \subset \mathfrak{s}^{+}$(resp. $\overline{\mathfrak{n}}^{-} \subset \mathfrak{s}^{-}$) the subspace that corresponds to $\overline{\mathfrak{u}}_{0}$. Set $\mathfrak{r}=\mathfrak{r}^{+} \oplus \mathfrak{r}^{-}, \mathfrak{n}=\mathfrak{n}^{+} \oplus \mathfrak{n}^{-}$and $\overline{\mathfrak{n}}=\overline{\mathfrak{n}}^{+} \oplus \overline{\mathfrak{n}}^{-}$. Then $\mathfrak{s}=\mathfrak{r} \oplus \mathfrak{n} \oplus \overline{\mathfrak{n}}$ and $X \in \mathfrak{r}(F)$. Notice that $\mathfrak{r}$ is isomorphic to a product of $\mathfrak{s}_{n_{i}}$ with $\sum n_{i}=n$. Also notice that $\mathfrak{n}^{\perp}=\mathfrak{r} \oplus \mathfrak{n}$ and $(\mathfrak{r} \oplus \mathfrak{n})^{\perp}=\mathfrak{n}$ under the fixed pairing $\langle\cdot, \cdot\rangle$ on $\mathfrak{s}$.

We call a subspace $\mathfrak{f}$ of $\mathfrak{s}$ a proper Levi subspace if $\mathfrak{f}$ is of the form $\mathfrak{r}$ as above for some $\mathfrak{r}$.

Let $\mathbf{P}=\mathbf{P}_{0} \times \mathbf{P}_{0}$, which is a parabolic subgroup of $\mathbf{H}=\mathrm{GL}_{n} \times \mathrm{GL}_{n}$. There is a Levi decomposition $\mathbf{P}=\mathbf{M U}$ and $\mathfrak{p}=\mathfrak{m} \oplus \mathfrak{u}$, with $\mathbf{M}=\mathbf{M}_{0} \times \mathbf{M}_{0}$, $\mathbf{U}=\mathbf{U}_{0} \times \mathbf{U}_{0}, \mathfrak{m}=\mathfrak{m}_{0} \oplus \mathfrak{m}_{0}$ and $\mathfrak{u}=\mathfrak{u}_{0} \oplus \mathfrak{u}_{0}$. Notice that $(\mathbf{M}, \mathfrak{r}) \simeq \prod\left(\mathbf{H}_{n_{i}}, \mathfrak{s}_{n_{i}}\right)$ for some $\left(\mathbf{H}_{n_{i}}, \mathfrak{s}_{n_{i}}\right)$. We fix an open compact subgroup $K$ of $H$ such that $H=M U K$ and $\left.\eta\right|_{K}$ is trivial. Recall that we write $M=\mathbf{M}(F)$ and $U=\mathbf{U}(F)$. Here we choose the Haar measure on $H$ so that $\operatorname{vol}(K)=1$, and choose Haar measures on $M$ and $U$ so that for any $f \in \mathcal{C}_{c}^{\infty}(H)$,

$$
\int_{H} f(h) \mathrm{d} h=\int_{M} \int_{U} \int_{K} f(m u k) \mathrm{d} m \mathrm{~d} u \mathrm{~d} k .
$$

We choose the Haar measure on Lie algebra $\mathfrak{u}(F)$ compatible with that on $U$ under the exponential map, and choose Haar measures on $\mathfrak{r}(F), \mathfrak{n}(F), \overline{\mathfrak{n}}(F)$ according to the above identifications.

For $f \in \mathcal{C}_{c}^{\infty}(\mathfrak{s}(F))$, we define $f^{\mathfrak{r}} \in \mathcal{C}_{c}^{\infty}(\mathfrak{r}(F))$ to be

$$
f^{\mathfrak{r}}(Y):=\int_{\mathfrak{n}(F)} f(Y+Z) \mathrm{d} Z,
$$

define $\tilde{f} \in \mathcal{C}_{c}^{\infty}(\mathfrak{s}(F))$ to be

$$
\tilde{f}(Y)=\int_{K} f\left(Y^{k}\right) \mathrm{d} k,
$$

and define $f^{(\mathfrak{r})} \in \mathcal{C}_{c}^{\infty}(\mathfrak{r}(F))$ to be

$$
f^{(\mathfrak{r})}:=(\widetilde{f})^{\mathfrak{r}}
$$

Definition 6.2. Let $T_{\mathfrak{r}}$ be a distribution on $\mathfrak{r}(F)$. We define the distribution $i_{\mathfrak{r}}\left(T_{\mathfrak{r}}\right)$ on $\mathfrak{s}(F)$ to be:

$$
i_{\mathfrak{r}}^{\mathfrak{s}}\left(T_{\mathfrak{r}}\right)(f):=T_{\mathfrak{r}}\left(f^{(\mathfrak{r})}\right), \quad \text { for } f \in \mathcal{C}_{c}^{\infty}(\mathfrak{s}(F)) .
$$


The above process is an analogue of parabolic induction in the usual sense (cf. [HC2, §1] or [Ko, §13]), and has the following similar properties. Notice that $\mathbf{M}$ acts on $\mathfrak{r}$ by the adjoint action, which is induced from the action of $\mathbf{H}$ on $\mathfrak{s}$. Denote by $\mathfrak{r}_{\mathrm{rs}}$ the regular semisimple locus of $\mathfrak{r}$ with respect to the action of $\mathbf{M}$. If $Y$ is in $\mathfrak{s}_{\mathrm{rs}}(F)$, then it is also in $\mathfrak{r}_{\mathrm{rs}}(F)$. If $Y \in \mathfrak{r}_{\mathrm{rs}}(F)$, put

$$
\left|D^{\mathfrak{r}}(Y)\right|_{F}=|\operatorname{det}(\operatorname{ad}(Y) ; \mathfrak{m} / \mathfrak{t} \oplus \mathfrak{r} / \mathfrak{c})|^{\frac{1}{2}}
$$

where $\mathfrak{c}$ is the Cartan space of $\mathfrak{r}$ containing $Y$ and $\mathfrak{t}$ is the Lie algebra of the centralizer of $Y$ in $\mathbf{M}$. The normalized orbital integral $I_{X}^{\eta, M}\left(f^{\prime}\right)$, for $f^{\prime} \in \mathcal{C}_{c}^{\infty}(\mathfrak{r}(F))$, is defined to be

$$
\left|D^{\mathfrak{r}}(X)\right|_{F}^{\frac{1}{2}} \int_{H_{X} \backslash M} f^{\prime}\left(X^{m}\right) \eta(m) \mathrm{d} m .
$$

Then $I_{X}^{\eta, M}$ is a distribution on $\mathfrak{r}(F)$. In the proposition below, we write $I_{X}^{\eta, H}$ instead of $I_{X}^{\eta}$ to distinguish it from $I_{X}^{\eta, M}$.

Proposition 6.3. 1. Suppose that $T_{\mathfrak{r}}$ is an $(M, \eta)$-invariant distribution on $\mathfrak{r}(F)$, then $i_{\mathfrak{r}}^{\mathfrak{s}}\left(T_{\mathfrak{r}}\right)$ is an $(H, \eta)$-invariant distribution on $\mathfrak{s}(F)$.

2. We have $i_{\mathfrak{r}}^{\mathfrak{s}}\left(I_{X}^{\eta, M}\right)=I_{X}^{\eta, H}$.

3. Suppose that $T_{\mathfrak{r}}$ is an $(M, \eta)$-invariant distribution on $\mathfrak{r}(F)$, which is represented by a function $\Theta_{\mathfrak{r}}$ which is locally constant on $\mathfrak{r}_{\mathrm{rs}}(F)$ and locally integrable on $\mathfrak{r}(F)$. In other words, for any $f \in \mathcal{C}_{c}^{\infty}(\mathfrak{r}(F))$,

$$
T_{\mathfrak{r}}(f)=\int_{\mathfrak{r}_{\mathrm{rs}}(F)} \Theta_{\mathfrak{r}}(Y) \kappa(Y) f(Y)\left|D^{\mathfrak{r}}(Y)\right|_{F}^{-1 / 2} \mathrm{~d} Y .
$$

Then the distribution $i_{\mathfrak{r}}\left(T_{\mathfrak{r}}\right)$ is represented by the function

$$
\Theta_{\mathfrak{s}}(Y)=\sum_{Y^{\prime}} \Theta_{\mathfrak{r}}\left(Y^{\prime}\right)
$$

where $Y^{\prime}$ runs over a finite set of representatives for the $M$-conjugacy classes of elements in $\mathfrak{r}(F)$ which are $H$-conjugate to $Y$. The function $\Theta_{\mathfrak{s}}$ is locally constant on $\mathfrak{s}_{\mathrm{rs}}(F)$ and locally integrable on $\mathfrak{s}(F)$, and, for any $f \in \mathcal{C}_{c}^{\infty}(\mathfrak{s}(F))$,

$$
i_{\mathfrak{r}}^{\mathfrak{s}}\left(T_{\mathfrak{r}}\right)(f)=\int_{\mathfrak{s}_{\mathrm{rs}}(F)} \Theta_{\mathfrak{s}}(Y) \kappa(Y) f(Y)\left|D^{\mathfrak{s}}(Y)\right|_{F}^{-1 / 2} \mathrm{~d} Y .
$$

4. The map $f \mapsto f^{(\mathfrak{r})}$ commutes with the Fourier transform, and therefore $i_{\mathfrak{r}}\left(\widehat{T}_{\mathfrak{r}}\right)=\widehat{i_{\mathfrak{r}}\left(T_{\mathfrak{r}}\right)}$.

Proof. (1) For $f \in \mathcal{C}_{c}^{\infty}(\mathfrak{s}(F))$ and $h \in H$, define ${ }^{h} f \in \mathcal{C}_{c}^{\infty}(\mathfrak{s}(F))$ by ${ }^{h} f(Y)=$ $f\left(Y^{h}\right)$. To prove (i), it suffices to observe the following relation: for $p=m u \in P$ and $Y \in \mathfrak{r}(F)$, we have

$$
\begin{aligned}
\left({ }^{p} f\right)^{\mathfrak{r}}(Y) & =\int_{\mathfrak{n}(F)}{ }^{p} f(Y+Z) \mathrm{d} Z=\int_{\mathfrak{n}(F)} f\left(Y^{p}+Z^{p}\right) \mathrm{d} Z \\
& =\int_{\mathfrak{n}(F)} f\left(Y^{m}+Z^{p}\right) \mathrm{d} Z \\
& =|\operatorname{det}(\operatorname{Ad}(p) ; \mathfrak{n})|_{F} \int_{\mathfrak{n}(F)} f\left(Y^{m}+Z\right) \mathrm{d} Z
\end{aligned}
$$


It is easy to verify that

$$
|\operatorname{det}(\operatorname{Ad}(p) ; \mathfrak{n})|_{F}=|\operatorname{det}(\operatorname{Ad}(p) ; \mathfrak{u})|_{F}=\delta_{P}(p),
$$

where $\delta_{P}$ is the modulus character of $P$. Therefore $\left({ }^{p} f\right)^{\mathfrak{r}}(Y)=\delta_{P}(p) f^{\mathfrak{r}}\left(Y^{m}\right)$. The rest arguments are routine.

(2). Write $T=H_{X}$ for simplicity. For $f \in \mathcal{C}_{c}^{\infty}(\mathfrak{s}(F))$,

$$
\begin{aligned}
\int_{T \backslash H} f\left(X^{h}\right) \eta(h) \mathrm{d} h & =\int_{T \backslash M} \int_{U} \int_{K} f\left(X^{m u k}\right) \eta(m) \mathrm{d} k \mathrm{~d} u \mathrm{~d} m \\
& =\int_{T \backslash M} \int_{U} \tilde{f}\left(X^{m u}\right) \eta(m) \mathrm{d} u \mathrm{~d} m .
\end{aligned}
$$

Write $Y=X^{m}$. Notice that the map

$$
\alpha: \mathbf{U} \longrightarrow \mathfrak{n}, \quad u \mapsto u^{-1} Y u-Y
$$

is an isomorphism of algebraic varieties, whose Jacobian is

$$
|\operatorname{det}(\varrho \circ \operatorname{ad}(Y) ; \mathfrak{u})|_{F} .
$$

Also note that

$$
\begin{aligned}
|\operatorname{det}(\varrho \circ \operatorname{ad}(Y) ; \mathfrak{u})|_{F} & =|\operatorname{det}(\varrho \circ \operatorname{ad}(Y) ; \mathfrak{u} \oplus \overline{\mathfrak{u}})|_{F}^{1 / 2} \\
& =\frac{|\operatorname{det}(\varrho \circ \operatorname{ad}(Y) ; \mathfrak{h} / \mathfrak{t})|_{F}^{1 / 2}}{|\operatorname{det}(\varrho \circ \operatorname{ad}(Y) ; \mathfrak{m} / \mathfrak{t})|_{F}^{1 / 2}} \\
& =\frac{\left|D^{\mathfrak{s}}(Y)\right|_{F}^{1 / 2}}{\left|D^{\mathfrak{r}}(Y)\right|_{F}^{1 / 2}} .
\end{aligned}
$$

Therefore

$$
\begin{aligned}
I^{\eta, H}(X, f) & =\int_{T \backslash M} \int_{\mathfrak{n}(F)}\left|D^{\mathfrak{r}}\left(X^{m}\right)\right|_{F}^{1 / 2} \widetilde{f}\left(X^{m}+Z\right) \eta(m) \mathrm{d} Z \mathrm{~d} m \\
& =\left|D^{\mathfrak{r}}(X)\right|_{F}^{1 / 2} \int_{T \backslash M} f^{(\mathfrak{r})}(X) \eta(m) \mathrm{d} m \\
& =I^{\eta, M}\left(X, f^{(\mathfrak{r})}\right) .
\end{aligned}
$$

The assertion (iii) is a consequence of Weyl integration formula, and the assertion (iv) is obvious.

Let $\mathfrak{s}_{\text {ell }}$ be the open subset of elliptic regular semisimple elements in $\mathfrak{s}(F)$.

Lemma 6.4. Suppose that $\phi \in \mathcal{C}_{c}^{\infty}\left(\mathfrak{s}_{\mathrm{ell}}\right)$. Then $\phi^{(\mathfrak{r})}$ and $(\widehat{\phi})^{(\mathfrak{r})}$ are identically zero for every proper Levi subspace $\mathfrak{r}$ of $\mathfrak{s}$. Moreover, for any regular semisimple element $X$ of $\mathfrak{s}(F)$ lying in $\mathfrak{r}(F)$, we have

$$
I^{\eta}(X, \phi)=\widehat{I}^{\eta}(X, \phi)=0 .
$$

Proof. The vanishing of $\phi^{(\mathfrak{r})}$ is obvious. The vanishing of $(\widehat{\phi})^{(\mathfrak{r})}$ is a consequence of Proposition 6.3 (iv). The vanishing of the orbital integrals follows from the first assertion and Proposition 6.3 (ii). 
Let $\mathfrak{c}$ be an elliptic Cartan subspace of $\mathfrak{s}$, which means that one (any) element of $\mathfrak{c}_{\text {reg }}(F)$ is elliptic. Let $T$ be the centralizer of $\mathfrak{c}$ in $H$, and $Z$ the center of $G$ which is also contained in $T$. Then $Z \backslash T$ is compact since $\mathfrak{c}$ is elliptic. Now we require that $\operatorname{vol}(Z \backslash T)=1$ here, which does not matter.

Let $\mathfrak{s}_{\mathrm{rs}}^{\mathfrak{c}}=\left(\mathfrak{c}_{\mathrm{reg}}(F)\right)^{H}$ and $\phi \in \mathcal{C}_{c}^{\infty}\left(\mathfrak{s}_{\mathrm{rs}}^{\mathfrak{c}}\right)$. We define the distribution $I_{\phi} \in$ $\mathcal{D}(\mathfrak{s}(F))^{H, \eta}$ to be

$$
I_{\phi}(f)=\int_{Z \backslash H} \int_{\mathfrak{s}(F)} f(Y) \phi\left(Y^{h}\right) \eta(h) \mathrm{d} Y \mathrm{~d} h .
$$

This distribution is well defined:

$$
\begin{aligned}
& \int_{Z \backslash H} \int_{\mathfrak{s}(F)}\left|f(Y) \phi\left(Y^{h}\right)\right| \mathrm{d} Y \mathrm{~d} h \\
= & \int_{Z \backslash H} \mathrm{~d} h\left(\int_{\mathfrak{c}(F)}\left|D^{\mathfrak{s}}(Y)\right|_{F} \mathrm{~d} Y \int_{Z \backslash H}\left|f\left(Y^{h^{\prime}}\right)\right| \cdot\left|\phi\left(Y^{h^{\prime} h}\right)\right| \mathrm{d} h^{\prime}\right) \\
= & \int_{\mathfrak{c}(F)}\left|D^{\mathfrak{s}}(Y)\right|_{F} \mathrm{~d} Y\left(\int_{(Z \backslash H) \times(Z \backslash H)}\left|f\left(Y^{h^{\prime}}\right)\right| \cdot\left|\phi\left(Y^{h}\right)\right| \mathrm{d} h^{\prime} \mathrm{d} h\right) \\
= & \int_{\mathfrak{c}_{\mathrm{reg}}(F)} I(Y,|f|) \cdot I(Y,|\phi|) \mathrm{d} Y<\infty,
\end{aligned}
$$

since $I(Y,|\phi|) \in \mathcal{C}_{c}^{\infty}\left(\mathfrak{c}_{\text {reg }}(F)\right)$. Here $I(\cdot, f)$ is the normalized orbital integral without twisting $\eta$. We also define the distribution $I_{\widehat{\phi}} \in \mathcal{D}(\mathfrak{s}(F))^{H, \eta}$ to be

$$
I_{\widehat{\phi}}(f)=\int_{Z \backslash H} \int_{\mathfrak{s}(F)} f(Y) \widehat{\phi}\left(Y^{h}\right) \eta(h) \mathrm{d} Y \mathrm{~d} h .
$$

We have the relation

$$
\int_{\mathfrak{s}(F)} f(Y) \widehat{\phi}\left(Y^{h}\right) \mathrm{d} Y=\int_{\mathfrak{s}(F)} \widehat{f}(Y) \phi\left(Y^{h}\right) \mathrm{d} Y .
$$

Thus

$$
\begin{aligned}
& \int_{Z \backslash H} \eta(h) \mathrm{d} h\left(\int_{\mathfrak{s}(F)} f(Y) \widehat{\phi}\left(Y^{h}\right) \mathrm{d} Y\right) \\
= & \int_{Z \backslash H} \eta(h) \mathrm{d} h\left(\int_{\mathfrak{s}(F)} \widehat{f}(Y) \phi\left(Y^{h}\right) \mathrm{d} Y\right),
\end{aligned}
$$

by the absolute convergence of the latter one, which shows that $I_{\widehat{\phi}}$ is well defined and $I_{\widehat{\phi}}=\widehat{I}_{\phi}$. In summary, we have the following lemma.

Lemma 6.5. Let $\mathfrak{c}$ be an elliptic Cartan subspace of $\mathfrak{s}$ and $\phi \in \mathcal{C}_{c}^{\infty}\left(\mathfrak{s}_{\mathrm{rs}}^{\mathfrak{c}}\right)$. Then $\widehat{I}_{\phi}=I_{\widehat{\phi}}$

In the next subsection, we will reduce Theorem 6.6 to the following theorem whose proof will be given in $\S \S 6.3-6.4$.

Theorem 6.6. Let $\mathfrak{c}$ be an elliptic Cartan subspace of $\mathfrak{s}$ and $\phi \in \mathcal{C}_{c}^{\infty}\left(\mathfrak{s}_{\mathrm{rs}}^{\mathfrak{c}}\right)$. Then $\widehat{I}_{\phi}$ is represented by a locally integrable function on $\mathfrak{s}(F)$ which is locally constant on $\mathfrak{s}_{\mathrm{rs}}(F)$. 


\subsection{Proof of Theorem 6.1}

To show the representability of the Fourier transform of orbital integrals, we need the following relative version of Howe's finiteness theorem (Theorem 6.7). Let us introduce some notation. If $\omega$ is a compact set in $\mathfrak{s}(F)$, put

$$
\mathcal{J}(\omega)^{\eta}=\left\{T \in \mathcal{D}(\mathfrak{s}(F))^{H, \eta}: \operatorname{Supp}(T) \subset \operatorname{cl}\left(\omega^{H}\right)\right\} .
$$

Let $L \subset \mathfrak{s}(F)$ be a lattice (a compact open $\mathcal{O}_{F}$-submodule). Denote by $\mathcal{C}_{c}(\mathfrak{s}(F) / L)$ the space of $f \in \mathcal{C}_{c}^{\infty}(\mathfrak{s}(F))$ which is invariant under translation by $L$. Let $j_{L}: \mathcal{J}(\omega)^{\eta} \rightarrow \mathcal{C}_{c}(\mathfrak{s}(F) / L)^{*}$ be the composition of the maps:

$$
j_{L}: \mathcal{J}(\omega)^{\eta} \hookrightarrow \mathcal{D}(\mathfrak{s}(F)) \stackrel{\text { res }}{\longrightarrow} \mathcal{C}_{c}(\mathfrak{s}(F) / L)^{*},
$$

where $\mathcal{C}_{c}(\mathfrak{s}(F) / L)^{*}$ is the vector space dual to $\mathcal{C}_{c}(\mathfrak{s}(F) / L)$ and res is the restriction map. Then Howe's finiteness theorem is the following.

Theorem 6.7. For any lattice $L$ and any compact set $\omega$ in $\mathfrak{s}(F)$, we have

$$
\operatorname{dim} j_{L}\left(\mathcal{J}(\omega)^{\eta}\right)<+\infty
$$

Proof. It was shown in [RR, Theorem 6.1] that Howe's finiteness theorem holds in a more general setting when $\eta=\mathbf{1}$. It is not hard to check that it still holds when $\eta$ is our quadratic character.

The following variant of Howe's theorem is often used, and we refer the reader to $[\mathrm{Ko}, \S 26]$ for more details. Let $\widehat{j}_{L}: \mathcal{J}(\omega)^{\eta} \rightarrow \mathcal{D}(L)$ be the composition of the maps

$$
\widehat{j}_{L}: \mathcal{J}(\omega)^{\eta} \hookrightarrow \mathcal{D}(\mathfrak{s}(F)) \stackrel{\mathcal{F}}{\longrightarrow} \mathcal{D}(\mathfrak{s}(F)) \stackrel{\text { res }}{\longrightarrow} \mathcal{D}(L),
$$

where $\mathcal{F}$ denotes the Fourier transform.

Theorem 6.8. For any lattice $L$ and any compact set $\omega$ in $\mathfrak{s}(F)$,

$$
\operatorname{dim} \widehat{j}_{L}\left(\mathcal{J}(\omega)^{\eta}\right)<+\infty .
$$

Proof. See [Ko, Theorem 26.3].

Corollary 6.9. Let $\omega$ be compact, and let $V$ be a subspace of $\mathcal{J}(\omega)^{\eta}$. Let $L$ be any lattice in $\mathfrak{s}(F)$. Then $\widehat{j}_{L}(V)=\widehat{j}_{L}(\operatorname{cl}(V))$.

Proof. See [Ko, Proposition 26.1].

Proof of Theorem 6.1. By Proposition 6.3, it suffices to show that $\widehat{I}_{X}^{\eta}$ can be represented when $X$ lies in $\mathfrak{c}_{\text {reg }}(F)$ for some elliptic Cartan subspace $\mathfrak{c}$ of $\mathfrak{s}$. Then Theorem 6.1 follows from Theorem 6.6, Lemma 6.10 and the fact that $\mathfrak{s}(F)=\bigcup_{\text {lattice }} L$.

Lemma 6.10. Let $X \in \mathfrak{c}_{\mathrm{reg}}(F)$ be an elliptic element and $\omega$ a compact open neighborhood of $X$ in $\mathfrak{c}_{\text {reg }}(F)$. Then given a lattice $L$ in $\mathfrak{s}(F)$, there exists $\phi \in \mathcal{C}_{c}^{\infty}\left(\omega^{H}\right)$ such that $\widehat{I}_{X}^{\eta}$ and $\widehat{I}_{\phi}$ have the same restriction to $L$. 
Proof. The proof is similar as that of [Ko, Lemma 26.5]. We first show that $I_{X}^{\eta}$ lies in the closure of the linear space

$$
I_{\omega}:=\left\{I_{\phi}: \phi \in \mathcal{C}_{c}^{\infty}\left(\omega^{H}\right)\right\},
$$

which is a subspace of $\mathcal{J}(\omega)^{\eta}$. It suffices to show that: if $I_{\phi}(f)=0$ for all $\phi \in \mathcal{C}_{c}^{\infty}\left(\omega^{H}\right)$ then $I_{X}^{\eta}(f)=0$. Note that

$$
I_{\phi}(f)=\int_{\omega} I_{Y}^{\eta}(f) \cdot I_{Y}^{\eta}(\phi) \mathrm{d} Y .
$$

We may shrink $\omega$ so that every function $\varphi \in \mathcal{C}_{c}^{\infty}(\omega)$ arises as $Y \mapsto I_{Y}^{\eta}(\phi)$ for some $\phi \in \mathcal{C}_{c}^{\infty}(\omega)$. Thus $I_{X}^{\eta}(f)=0$ if $I_{\phi}(f)=0$ for all $\phi \in \mathcal{C}_{c}^{\infty}\left(\omega^{H}\right)$. By Corollary 6.9 , we see that $\hat{j}_{L}\left(I_{X}^{\eta}\right) \in \widehat{j}_{L}\left(I_{\omega}\right)$ for any lattice $L$. In other words, given a lattice $L$, there exists a $\phi \in \mathcal{C}_{c}^{\infty}\left(\omega^{H}\right)$ such that $\widehat{I}_{X}^{\eta}$ and $\widehat{I}_{\phi}$ have the same restriction to $L$.

\subsection{Bounding the orbital integrals}

In this subsection, we will show the boundness of the normalized orbital integrals along a Cartan subspace (Theorem 6.11), which is crucial for proving Theorem 6.6. We follow the same line as the proof of [HC1, Theorem 14], where there are no Shalika germs involved.

Theorem 6.11. 1. Let $\mathfrak{c}$ be a Cartan subspace of $\mathfrak{s}$ and $f \in \mathcal{C}_{c}^{\infty}(\mathfrak{s}(F))$. Then

$$
\sup _{X \in \mathfrak{c}_{\text {reg }}(F)}\left|I^{\eta}(X, f)\right|<+\infty .
$$

2. Let $\mathfrak{c}^{\prime}$ be a Cartan subspace of $\mathfrak{s}^{\prime}$ and $f^{\prime} \in \mathcal{C}_{c}^{\infty}\left(\mathfrak{s}^{\prime}(F)\right)$. Then

$$
\sup _{X \in \mathfrak{c}_{\text {reg }}^{\prime}(F)}\left|I\left(X, f^{\prime}\right)\right|<+\infty .
$$

We will prove only the first assertion with respect to $\mathfrak{s}$. The second assertion can be proved in the same way. We use inductive method to prove this theorem. In the case $n=1$, our case essentially is the Gan-Gross-Prasad conjecture for unitary groups of rank 1. Thus Theorem 6.11 follows from the discussions in [Zhw1, §4.1] (in particular, Lemma 4.1 in loc. cit.). Now we assume that Theorem 6.11 holds for $\mathcal{C}_{c}^{\infty}\left(\mathfrak{s}_{m}(F)\right)$ for every $m<n$.

Lemma 6.12. Fix a compact set $\omega$ of $\mathfrak{s}(F)$ and a Cartan subspace $\mathfrak{c}$. Then the set of all $X \in \mathfrak{c}(F)$ such that $X \in \operatorname{cl}\left(\omega^{H}\right)$ is relative compact in $\mathfrak{c}(F)$.

Proof. It suffices to assume $\omega$ is closed. Consider the closed inclusion $i$ : $(\mathfrak{c} / W)(F) \rightarrow(\mathfrak{s} / \mathbf{H})(F)$ where $W$ is the Weyl group of $\mathfrak{c}$, and the natural map $\pi: \mathfrak{s}(F) \rightarrow(\mathfrak{s} / \mathbf{H})(F)$. Then $\pi(\omega)$ and thus $i^{-1}(\pi(\omega))$ is compact. The lemma follows from the fact that the map $\mathfrak{c}(F) \rightarrow(\mathfrak{c} / W)(F)$ is a proper map between locally compact Hausdorff spaces.

Corollary 6.13. For $f \in \mathcal{C}_{c}^{\infty}(\mathfrak{s}(F)), I^{\eta}(X, f)=0$ for $X \in \mathfrak{c}_{\text {reg }}(F)$ lying outside a compact subset of $\mathfrak{c}(F)$.

We first prove Theorem 6.11 in the following situation. 
Lemma 6.14. Let $f$ be in $\mathcal{C}_{c}^{\infty}(\mathfrak{s}(F)-\mathcal{N})$. Then $I^{\eta}(\cdot, f)$ is bounded on $\mathfrak{c}_{\text {reg }}(F)$.

Proof. By Lemma 6.12 and Corollary 6.13, it suffices to prove: given $X_{0} \in \mathfrak{c}(F)$, we can choose a neighborhood $V$ of $X_{0}$ in $\mathfrak{c}(F)$ such that

$$
\sup _{X \in V^{\prime}}\left|I^{\eta}(X, f)\right|<+\infty, \quad \text { where } V^{\prime}=V \cap \mathfrak{s}_{\mathrm{rs}}(F) .
$$

When $X_{0} \neq 0$, using the descent of orbital integrals (Proposition 5.20), we reduce to considering the orbital integrals for $\mathcal{C}_{c}^{\infty}\left(\mathfrak{s}_{X_{0}}\right)$ with respect to the action of $H_{X_{0}}$. Since $X_{0} \neq 0,\left(H_{X_{0}}, \mathfrak{s}_{X_{0}}\right)$ is of the form

$$
\left(\mathrm{GL}_{m}(F)_{A}, \mathfrak{g l}_{m}(F)_{A}\right) \times\left(H_{n-m}, \mathfrak{s}_{n-m}(F)\right)
$$

for some semisimple $A$ in $\operatorname{GL}_{n}(F)$ and some integer $0<m \leq n$. Then the result follows from the inductive hypothesis on $n-m$ and the bound of the usual orbital integrals for $\mathcal{C}_{c}^{\infty}\left(\mathfrak{g l}_{m}(F)_{A}\right)$ by Harish-Chandra. When $X_{0}=0$, since $\operatorname{Supp}(f) \cap \mathcal{N}=\emptyset$, we can find a neighborhood $V$ of $X_{0}$ such that $I^{\eta}(X, f)=0$ on $V^{\prime}$.

Now let $\mathfrak{s}_{0}$ be the set of $Y \in \mathfrak{s}(F)$ such that: there exists an open neighborhood $\omega$ of $Y$ in $\mathfrak{s}(F)$ so that $\sup _{X \in \mathfrak{c}_{\text {reg }}(F)}\left|I^{\eta}(X, f)\right|<\infty$ for all $f \in \mathcal{C}_{c}^{\infty}(\mathfrak{s}(F))$ with $\operatorname{Supp}(f) \subset \omega$. Since $\mathcal{N}$ is closed in $\mathfrak{s}(F)$, Lemma 6.14 implies that $\mathfrak{s}(F)-\mathcal{N} \subset \mathfrak{s}_{0}$. To prove Theorem 6.11 , it remains to show that $\mathcal{N} \subset \mathfrak{s}_{0}$. We need some preparation below.

Fix $X_{0} \neq 0$ in $\mathcal{N}$. Let $\left(X_{0}, \mathrm{~d}\left(X_{0}\right), Y_{0}\right)$ be an $\mathfrak{s l}_{2}$-triple as in Lemma 3.1. Consider the map

$$
\psi: H \times \mathfrak{s}_{Y_{0}} \longrightarrow \mathfrak{s}(F), \quad(h, U) \mapsto\left(X_{0}+U\right)^{h} .
$$

By the same discussion as that of [HC1, Part VI, $\S 4]$, we see that $\psi$ is everywhere submersive. Set $\omega=\psi\left(H \times \mathfrak{s}_{Y_{0}}\right)$, which is an open and $H$-invariant subset of $\mathfrak{s}(F)$. Since $\psi$ is everywhere submersive, we have a surjective linear map

$$
\mathcal{C}_{c}^{\infty}\left(H \times \mathfrak{s}_{Y_{0}}\right) \longrightarrow \mathcal{C}_{c}^{\infty}(\omega), \quad \alpha \mapsto f_{\alpha}
$$

such that

$$
\int_{\omega} f_{\alpha}(X) p(X) \mathrm{d} X=\int_{H \times \mathfrak{s}_{Y_{0}}} \alpha(h, u) p\left(\left(X_{0}+U\right)^{h}\right) \mathrm{d} h \mathrm{~d} U
$$

for every locally integrable function $p$ on $\omega$.

Let $\Gamma$ be the Cartan subgroup of $H$ with the Lie algebra $F \cdot \mathrm{d}\left(X_{0}\right)$. Please refer to Lemma 3.3 and Proposition 4.4 for the notations below. Put $t=\xi(\gamma)$ and write $U_{\gamma}=\xi(\gamma) U^{\gamma^{-1}}$ for $U \in \mathfrak{s}_{Y_{0}}, \gamma \in \Gamma$. We have

$$
\left(X_{0}+U_{\gamma}\right)^{\gamma h}=\left(X_{0}+t U^{\gamma^{-1}}\right)^{\gamma h}=t\left(X_{0}+U\right)^{h} .
$$

For $\gamma \in \Gamma$ and $\alpha \in \mathcal{C}_{c}^{\infty}\left(H \times \mathfrak{s}_{Y_{0}}\right)$, define $\alpha^{\prime} \in \mathcal{C}_{c}^{\infty}\left(H \times \mathfrak{s}_{Y_{0}}\right)$ to be

$$
\alpha^{\prime}(h, U)=\alpha\left(\gamma^{-1} h, U_{\gamma^{-1}}\right)
$$


Lemma 6.15. Fix $\gamma \in \Gamma$ and $\alpha \in \mathcal{C}_{c}^{\infty}\left(H \times \mathfrak{s}_{Y_{0}}\right)$. Then

$$
f_{\alpha}\left(t^{-1} X\right)=|t|_{F}^{2 n^{2}-r-m} f_{\alpha^{\prime}}(X), \quad X \in \omega .
$$

Proof. Choose any function $\alpha$ in $\mathcal{C}_{c}^{\infty}(\omega)$. We have

$$
\begin{aligned}
& \int_{\mathfrak{s}(F)} f_{\alpha}\left(t^{-1} X\right) p(X) \mathrm{d} X \\
= & |t|_{F}^{2 n^{2}} \int_{\mathfrak{s}(F)} f_{\alpha}(X) p(t X) \mathrm{d} X \\
= & |t|_{F}^{2 n^{2}} \int_{H \times \mathfrak{s}_{Y_{0}}} \alpha(h, U) p\left(t\left(X_{0}+U\right)^{h}\right) \mathrm{d} h \mathrm{~d} U \\
= & |t|_{F}^{2 n^{2}} \int_{H \times \mathfrak{s}_{Y_{0}}} \alpha(h, U) p\left(\left(X_{0}+U_{\gamma}\right)^{\gamma h}\right) \mathrm{d} h \mathrm{~d} U \\
= & |t|_{F}^{2 n^{2}} \int_{H \times \mathfrak{s}_{Y_{0}}} \alpha\left(\gamma^{-1} h, U_{\gamma^{-1}}\right) p\left(\left(X_{0}+U\right)^{h}\right)\left|\frac{\mathrm{d} U_{\gamma^{-1}}}{\mathrm{~d} U}\right|_{F} \mathrm{~d} h \mathrm{~d} U .
\end{aligned}
$$

It remains to compute the Jacobian $\left|\frac{\mathrm{d} U_{\gamma-1}}{\mathrm{~d} U}\right|_{F}$. Choose a basis $U_{1}, \ldots, U_{r}$ of $\mathfrak{s}_{Y_{0}}$ as in Lemma 3.3. Write $U=\sum_{1 \leq i \leq r} a_{i} U_{i}$. Then

$$
\begin{aligned}
U_{\gamma^{-1}} & =t^{-1} U^{\gamma}=t^{-1} \sum_{i} a_{i} U_{i}^{\gamma} \\
& =t^{-1} \sum_{i} a_{i} \xi_{i}\left(\gamma^{-1}\right) U_{i} .
\end{aligned} .
$$

Hence

$$
\left|\frac{\mathrm{d} U_{\gamma^{-1}}}{\mathrm{~d} U}\right|_{F}=|t|_{F}^{-r} \prod_{1 \leq i \leq r}|t|_{F}^{\frac{-\lambda_{i}}{2}}=|t|_{F}^{-r-m}
$$

which implies the lemma.

For $X \in \mathfrak{c}_{\text {reg }}(F)$, there is a unique distribution $\tau_{X}^{\eta}$ on $\mathfrak{s}_{Y_{0}}$ such that $I^{\eta}\left(X, f_{\alpha}\right)=$ $\tau_{X}^{\eta}\left(\beta_{\alpha}\right)$ where

$$
\beta_{\alpha}(U)=\int_{H} \alpha(h, U) \eta(h) \mathrm{d} h, \quad \alpha \in \mathcal{C}_{c}^{\infty}\left(H \times \mathfrak{s}_{Y_{0}}\right) .
$$

For $f \in \mathcal{C}_{c}^{\infty}(\omega)$, define $f^{\prime} \in \mathcal{C}_{c}^{\infty}(\omega)$ to be $f^{\prime}(X)=f\left(t^{-1} X\right)$. It is easy to see that

$$
I^{\eta}\left(X, f^{\prime}\right)=|t|_{F}^{\frac{1}{2}\left(2 n^{2}-n\right)} I^{\eta}\left(t^{-1} X, f\right) .
$$

Now fix $\alpha \in \mathcal{C}_{c}^{\infty}\left(H \times \mathfrak{s}_{Y_{0}}\right)$, and set $f=f_{\alpha}, f^{\prime}=f_{\alpha}^{\prime}, \beta=\beta_{\alpha}$ and $\beta^{\prime}=\beta_{\alpha^{\prime}}$. Note that

$$
f^{\prime}=|t|_{F}^{2 n^{2}-r-m} f_{\alpha^{\prime}}
$$

We have

$$
\beta^{\prime}(U)=\int_{H} \alpha\left(\gamma^{-1} h, U_{\gamma^{-1}}\right) \eta(h) \mathrm{d} h=\eta(\gamma) \beta\left(t^{-1} U^{\gamma}\right), \quad U \in \mathfrak{s}_{Y_{0}} .
$$

So we obtain

$$
|t|_{F}^{\frac{1}{2}\left(2 n^{2}-n\right)} I^{\eta}\left(t^{-1} X, f\right)=|t|_{F}^{2 n^{2}-r-m} I^{\eta}\left(X, f_{\alpha^{\prime}}\right)=|t|_{F}^{2 n^{2}-r-m} \tau_{X}^{\eta}\left(\beta^{\prime}\right),
$$


or

$$
I^{\eta}\left(t^{-1} X, f\right)=|t|_{F}^{n^{2}+\frac{n}{2}-r-m}\left(I^{\eta}(X, f)+\tau_{X}^{\eta}\left(\beta^{\prime}-\beta\right)\right) .
$$

By Proposition 4.4, we know $n^{2}+\frac{n}{2}-r-m<0$.

Now we continue to prove Theorem 6.11. Let $X_{0} \in \mathcal{N}$ and suppose $X_{0} \neq 0$. We want to construct an open neighborhood $\omega_{0}$ of $X_{0}$ such that $I^{\eta}(\cdot, f)$ is bounded on $\mathfrak{c}_{\text {reg }}(F)$ as soon as $\operatorname{Supp}(f) \subset \omega_{0}$. Recall that we denote by $\mathcal{N}_{q}$ the union of all $H$-orbits in $\mathcal{N}$ of dimension $\leq q$, and notice that $X_{0} \in \mathcal{N}_{2 n^{2}-r}$ and $\mathcal{N}_{2 n^{2}-n}=\mathcal{N}$. So we can choose an open neighborhood $\omega_{1}$ of $X_{0}$ in $\omega$ such that $\omega_{1} \cap \mathcal{N}_{2 n^{2}-r} \subset X_{0}^{H}$, and can assume $\omega_{1}=\omega_{1}^{H}$. By [HC1, Lemma 37], we can choose an open neighborhood $\mathcal{U}$ of zero in $\mathfrak{s}_{Y_{0}}$ such that $X_{0}+\mathcal{U} \subset \omega_{1}$ and $\left(X_{0}+\mathcal{U}\right) \cap X_{0}^{H}=\left\{X_{0}\right\}$.

Fix $\gamma \in \Gamma$ such that $\eta(\gamma)=1$ and $|t|_{F}=|\xi(\gamma)|_{F}>1$. Choose an open neighborhood $\mathcal{U}_{0}$ of zero in $\mathcal{U}$ such that $t^{-1} \mathcal{U}_{0}^{\gamma} \cup t \mathcal{U}_{0}^{\gamma}{ }^{-1} \subset \mathcal{U}$. Put $\mathcal{N}^{*}=\mathcal{N}-\{0\}$.

Lemma 6.16. $\mathcal{N}^{*} \subset \mathfrak{s}_{0}$.

Proof. We induct on $r=\operatorname{dim} \mathfrak{s}_{Y_{0}}$ for $X_{0} \in \mathcal{N}^{*}$. Put $\omega_{0}=\left(X_{0}+\mathcal{U}_{0}\right)^{H}$, which is an open invariant neighborhood of $X_{0}$. Consider the surjective map

$$
H \times \mathcal{U}_{0} \longrightarrow \omega_{0}, \quad(h, U) \mapsto\left(X_{0}+U\right)^{h},
$$

which is everywhere submersive. Consider the surjective linear map

$$
\mathcal{C}_{c}^{\infty}\left(H \times \mathcal{U}_{0}\right) \longrightarrow \mathcal{C}_{c}^{\infty}\left(\omega_{0}\right), \quad \alpha \mapsto f_{\alpha},
$$

which is the restriction of the $\operatorname{map} \mathcal{C}_{c}^{\infty}\left(H \times \mathfrak{s}_{Y_{0}}\right) \rightarrow \mathcal{C}_{c}^{\infty}(\omega)$ as before. Let $f \in \mathcal{C}_{c}^{\infty}\left(\omega_{0}\right)$ and choose $\alpha \in \mathcal{C}_{c}^{\infty}\left(H \times \mathcal{U}_{0}\right)$ such that $f=f_{\alpha}$. Set $\beta=\beta_{\alpha}, \beta^{\prime}=\beta_{\alpha^{\prime}}$ and $f^{\prime}=f_{\alpha}^{\prime}$ as before. Then $\beta-\beta^{\prime} \in \mathcal{C}_{c}^{\infty}(\mathcal{U})$, and $0 \notin \operatorname{Supp}\left(\beta-\beta^{\prime}\right)$. Define $\alpha_{0}(h, U)=\alpha_{1}(h)\left(\beta(U)-\beta^{\prime}(U)\right)$ for $h \in H, U \in \mathcal{U}$, where $\alpha_{1} \in \mathcal{C}_{c}^{\infty}(H)$ and $\int_{H} \alpha_{1}(h) \eta(h) \mathrm{d} h=1$. For $X \in \mathfrak{c}_{\text {reg }}(F)$, we have

$$
I^{\eta}\left(X, f_{\alpha_{0}}\right)=\tau_{X}^{\eta}\left(\beta_{\alpha_{0}}\right)=\tau_{X}^{\eta}\left(\beta-\beta^{\prime}\right),
$$

and $\operatorname{Supp}\left(f_{\alpha_{0}}\right) \cap \mathcal{N}_{2 n^{2}-r}=\emptyset$. Now we start the induction on $r=\operatorname{dim} \mathfrak{s}_{Y_{0}}$.

First assume that $r=n$. Note that $r=n$ is the initial step. In such case we have $n^{2}+\frac{n}{2}-r-m=-\frac{n}{2}$ and

$$
I^{\eta}\left(t^{-1} X, f\right)=|t|_{F}^{-\frac{n}{2}}\left(I^{\eta}(X, f)+\tau_{X}^{\eta}\left(\beta^{\prime}-\beta\right)\right),
$$

by (1). Put $c=|t|_{F}^{-\frac{n}{2}}<1$. Since $\operatorname{Supp}\left(f_{\alpha_{0}}\right) \cap \mathcal{N}=\emptyset\left(\mathcal{N}=\mathcal{N}_{2 n^{2}-n}\right)$, by Lemma 6.14 , we have

$$
a=\sup _{X \in \mathfrak{c}_{\text {reg }}(F)}\left|\tau_{X}^{\eta}\left(\beta^{\prime}-\beta\right)\right|<+\infty .
$$

Iteration gives

$$
I^{\eta}\left(t^{-d} X, f\right)=|t|_{F}^{-\frac{d n}{2}} I^{\eta}(X, f)+\sum_{1 \leq k \leq d}|t|_{F}^{-\frac{k d}{2}} \tau_{t^{k-d} X}^{\eta}\left(\beta^{\prime}-\beta\right), \quad(d \geq 1),
$$

or

$$
I^{\eta}(X, f)=c^{d} I^{\eta}\left(t^{d} X, f\right)+\sum_{1 \leq k \leq d} c^{d} \tau_{t^{k} X}^{\eta}\left(\beta^{\prime}-\beta\right), \quad(d \geq 1)
$$


Since $\lim _{d \rightarrow+\infty} I^{\eta}\left(t^{d} X, f\right)=0$, we get

$$
\left|I^{\eta}(X, f)\right| \leq a \sum_{1 \leq k<\infty} c^{k} \leq a \frac{c}{1-c} .
$$

Now assume $r>n$. Since $\operatorname{Supp}\left(f_{\alpha_{0}}\right) \cap \mathcal{N}_{2 n^{2}-r}=\emptyset$, by the inductive hypothesis and Lemma $6.14, I^{\eta}\left(X, f_{\alpha_{0}}\right)$ is bounded on $\mathfrak{c}_{\mathrm{reg}}(F)$ and so is $\tau_{X}^{\eta}\left(\beta-\beta^{\prime}\right)$. Applying the same argument as the case $r=n$, we complete the proof of the lemma.

Applying the same arguments as those of [HC1, Part VI $\S 7$, we have the following lemma.

Lemma 6.17. $0 \in \mathfrak{s}_{0}$.

At last, Theorem 6.11 follows from Lemma 6.14, Lemma 6.16 and Lemma 6.17 .

\subsection{Proof of Theorem 6.6}

Now we continue to prove Theorem 6.6. Let $\mathfrak{c}_{0}$ be an elliptic Cartan subspace of $\mathfrak{s}$ and $\phi_{0} \in \mathcal{C}_{c}^{\infty}\left(\mathfrak{s}_{\mathrm{rs}}^{\mathfrak{c}_{0}}\right)$. For simplicity, we write $\phi=\widehat{\phi_{0}}$, and denote by $\Theta$ the distribution $I_{\phi}$, that is, for $f \in \mathcal{C}_{c}^{\infty}(\mathfrak{s}(F))$,

$$
\Theta(f):=\int_{Z \backslash H} \int_{\mathfrak{s}(F)} f(Y) \phi\left(Y^{h}\right) \eta(h) \mathrm{d} Y \mathrm{~d} h .
$$

Our goal is to prove that the distribution $\Theta$ can be represented by a locally integrable function on $\mathfrak{s}(F)$ which is locally constant on $\mathfrak{s}_{\mathrm{rs}}(F)$. We follow the strategy of the proof of [HC1, Theorem 16].

For $t \geq 1$, let $\Omega_{t}$ denote the set of all $h \in H$ such that $1+\log \|h\|_{Z \backslash H} \leq t$. Then $\Omega_{t}$ is a compact set modulo $Z$. Let $\Phi_{t}$ denote the characteristic function of $\Omega_{t}$. Then we have

$$
\begin{aligned}
\Theta(f) & =\lim _{t \rightarrow+\infty} \int_{Z \backslash H} \Phi_{t}(h) \int_{\mathfrak{s}(F)} f(Y) \phi\left(Y^{h}\right) \eta(h) \mathrm{d} Y \mathrm{~d} h \\
& =\lim _{t \rightarrow+\infty} \int_{\mathfrak{s}(F)} f(Y) \Theta_{t}(Y) \mathrm{d} Y,
\end{aligned}
$$

where

$$
\Theta_{t}(Y)=\int_{Z \backslash H} \Phi_{t}(h) \phi\left(Y^{h}\right) \eta(h) \mathrm{d} h .
$$

We will first show that $\lim _{t \rightarrow+\infty} \Theta_{t}(Y)$ exists for all $Y \in \mathfrak{s}_{\mathrm{rs}}(F)$, and then will give an estimation on $\Theta_{t}$ to apply Lebesgue's Theorem.

Lemma 6.18. Given a compact subset $\omega$ of $\mathfrak{s}(F)$, we can choose $c_{0} \geq 0$ such that

$$
1+\log \|h\|_{T \backslash H} \leq c_{0}\left(1+\log \left(\max \left\{1,\left|D^{\mathfrak{s}}(X)\right|_{F}^{-1}\right\}\right)\right)
$$

for $h \in H, X \in \mathfrak{c}_{\mathrm{reg}}(F)$ such that $X^{h} \in \omega$.

Proof. The proof is the same as that of [Ko, Lemma 20.3]. 
We choose a compact set $\omega \subset \mathfrak{s}(F)$ such that $\operatorname{Supp}(\phi) \subset \omega, \operatorname{Supp}(f) \subset \omega$. Fix a Cartan subspace $\mathfrak{c} \subset \mathfrak{s}$. Let $T$ be the centralizer of $\mathfrak{c}$ in $H$, and $A$ the maximal split torus in $T$. Notice that $A$ consists of elements of the form $\operatorname{diag}(a, a)$ where $a \in A_{0}$ for some split torus $A_{0}$ contained in $\operatorname{GL}_{n}(F)$. Let $\omega_{\mathfrak{c}}$ be the set of $X \in \mathfrak{c}(F)$ such that $X^{h} \in \omega$ for some $h \in H$. Then $\omega_{\mathfrak{c}}$ is compact. For $X \in \omega_{\mathfrak{c}}, h \in H$, set

$$
\phi_{X}(h)=\phi\left(X^{h}\right) \eta(h) .
$$

Note that $\phi_{X}$ has the following properties:

(i) $\operatorname{Supp}\left(\phi_{X}\right) \subset C_{X}$ for some subset $C_{X} \subset H$ which is compact modulo $A$ and $\phi_{X}(a h)=\phi_{X}(h)$ for $h \in H, a \in A$;

(ii) if $P_{0}^{\prime}$ is a proper parabolic subgroup in $\mathrm{GL}_{n}(F)$ with Levi decomposition $P_{0}^{\prime}=M_{0}^{\prime} U_{0}^{\prime}$, and $A_{0}^{\prime} \subset A_{0}$ where $A_{0}^{\prime}$ is the center of $M_{0}^{\prime}$, then

$$
\int_{U^{\prime}} \phi_{X}(u h) \mathrm{d} u=0, \quad \text { for each } h \in H
$$

where $U^{\prime}=U_{0}^{\prime} \times U_{0}^{\prime}$ is a unipotent subgroup of $H$.

Let $K_{1}^{\prime}$ be an open subgroup of $K^{\prime}=\mathrm{GL}_{n}\left(\mathcal{O}_{F}\right)$ such that $\|k\|=1, \eta(k)=1$ for all $k \in K_{1}^{\prime}$. Here we choose the Haar measure on $H$ so that $\operatorname{vol}\left(K_{1}^{\prime} \times K_{1}^{\prime}\right)=1$. Fix an open compact subgroup $K_{0}^{\prime}$ of $\mathrm{GL}_{n}(F)$ such that

$$
K_{0}^{\prime} \subset\left(\bar{U} \cap K_{1}^{\prime}\right)\left(M \cap K_{1}^{\prime}\right)\left(U \cap K_{1}^{\prime}\right)
$$

for any parabolic subgroup $P^{\prime}=M^{\prime} U^{\prime}$ in $\mathcal{P}\left(A_{0}\right)$, where we denote by $\mathcal{P}\left(A_{0}\right)$ the set of all parabolic subgroups $P^{\prime}=M^{\prime} U^{\prime}$ of $\mathrm{GL}_{n}(F)$ such that $A_{0}$ is the center of $M^{\prime}$. Set $K_{0}=K_{0}^{\prime} \times K_{0}^{\prime} \subset H$. For an element $y \in H$, put $K_{0}(y)=K_{0} \cap K_{0}^{y}$. Set

$$
\left\|C_{X}\right\|_{T \backslash H}=\sup _{h \in C_{X}}\|h\|_{T \backslash H} .
$$

The following lemma is an analogue of [HC1, Theorem 20], and we omit the details of the proof since it is the same as that of [HC1, Theorem 20]

Lemma 6.19. There exists a number $c \geq 1$ with the following property. Let $y \in H$, and $\Omega=\Omega\left(C_{X}, y\right)$ be the set of $h \in H$ such that

$$
1+\log \|h\|_{Z \backslash H} \leq c\left(1+\log \left\|C_{X}\right\|_{T \backslash H}\right)\left(1+\log \|y\|_{T \backslash H}\right) .
$$

Then

$$
\int_{K_{0}(y)} \phi_{X}(y k h) \mathrm{d} k=0
$$

unless $h \in \Omega$.

Now suppose that $X \in \mathfrak{c}_{\mathrm{reg}}(F)$ and $y \in H$ are such that $X^{y} \in \omega$. Then $X \in \omega_{\mathfrak{c}}$. By Lemma 6.18, there is a positive constant $c_{0}$, only depending on $\omega$ and $\mathfrak{c}$, such that

$$
1+\log \|y\|_{T \backslash H} \leq c_{0}\left(1+\log \left(\max \left\{1,\left|D^{\mathfrak{s}}(X)\right|_{F}^{-1}\right\}\right)\right) .
$$

Set $\omega_{\mathfrak{c}}^{\prime}=\omega_{\mathfrak{c}} \cap \mathfrak{c}_{\text {reg }}(F)$. Then for any $X \in \omega_{\mathfrak{c}}^{\prime}$ we can choose a subset $C_{X}$ of $H$ such that

(1) $\operatorname{Supp}\left(\phi_{X}\right) \subset C_{X}$ and $C_{X}$ is compact modulo $A$; 
(2) $1+\log \left\|C_{X}\right\|_{T \backslash H} \leq c_{0}\left(1+\log \left(\max \left\{1,\left|D^{\mathfrak{s}}(X)\right|_{F}^{-1}\right\}\right)\right)$.

Let $\Omega_{X}\left(X \in \omega_{\mathfrak{c}}^{\prime}\right)$ be the set of $h \in H$ such that

$$
1+\log \|h\|_{Z \backslash H} \leq c_{1}\left(1+\log \left(\max \left\{1,\left|D^{\mathfrak{s}}(X)\right|_{F}^{-1}\right\}\right)\right)^{2},
$$

where $c_{1}=c \cdot c_{0}^{2}$ with $c$ as in Lemma 6.19 . Let $\Phi_{X}$ denote the characteristic function of $\Omega_{X}$. Then we have

$$
\begin{aligned}
\Theta_{t}\left(X^{y}\right) & =\int_{Z \backslash H} \Phi_{t}(h) \phi\left(X^{y h}\right) \eta(h) \mathrm{d} h \\
& =\int_{Z \backslash H} \Phi_{t}(h) \int_{K_{1}} \phi\left(X^{y k h}\right) \eta(h) \mathrm{d} k \mathrm{~d} h .
\end{aligned}
$$

Note that $\|k h\|=\|h\|$ for $k \in K_{1}$. By Lemma 6.19 we have

$$
\int_{K_{1}} \phi\left(X^{y k h}\right) \mathrm{d} k=\int_{K_{1}} \phi_{X}(y k h) \mathrm{d} k=0,
$$

unless:

$$
1+\log \left\|k_{1} h\right\|_{Z \backslash H} \leq c\left(1+\log \left\|C_{X}\right\|_{T \backslash H}\right)\left(1+\log \|y\|_{T \backslash H}\right),
$$

where $k_{1}$ runs over a set of representatives of $K_{1} / K_{0}\left(y_{0}\right)$ in $K_{1}$. Since $\|k h\|=$ $\|h\|$ and

$$
c\left(1+\log \left\|C_{X}\right\|_{T \backslash H}\right)\left(1+\log \|y\|_{T \backslash H}\right) \leq c c_{0}^{2}\left(1+\log \left(\max \left\{1,\left|D^{\mathfrak{s}}(X)\right|_{F}^{-1}\right\}\right)\right)^{2},
$$

the integral $\int_{K_{1}} \phi\left(X^{y k h}\right)=0$ unless $h \in \Omega_{X}$. Thus, if

$$
t \geq c_{1}\left(1+\log \left(\max \left\{1,\left|D^{\mathfrak{s}}(X)\right|_{F}^{-1}\right\}\right)\right)^{2},
$$

we get

$$
\begin{aligned}
\Theta_{t}\left(X^{y}\right) & =\int_{Z \backslash H} \Phi_{t}(h) \Phi_{X}(h) \eta(h) \int_{K_{1}} \phi\left(X^{y k h}\right) \mathrm{d} k \mathrm{~d} h \\
& =\int_{Z \backslash H} \Phi_{X}(h) \int_{K_{1}} \phi\left(X^{y k h}\right) \mathrm{d} k \mathrm{~d} h \\
& =\int_{Z \backslash H} \int_{K_{1}} \phi\left(X^{y k h}\right) \eta(h) \mathrm{d} k \mathrm{~d} h .
\end{aligned}
$$

Therefore $\lim _{t \rightarrow+\infty} \Theta_{t}\left(X^{y}\right)$ exists for $X^{y} \in \omega \cap \mathfrak{s}_{\mathrm{rs}}(F)$. By enlarging $\omega, \lim _{t \rightarrow+\infty} \Theta_{t}(X)$ exists for all $X \in \mathfrak{s}_{\mathrm{rs}}(F)$.

Now we estimate $\Theta_{t}(X)$. All the notations are the same as above. We have

$$
\begin{aligned}
\left|\Theta_{t}\left(X^{y}\right)\right| & \leq \int_{Z \backslash H} \Phi_{X}\left(y^{-1} h\right)\left|\phi\left(X^{h}\right)\right| \mathrm{d} h \\
& =\int_{A \backslash H}\left|\phi\left(X^{h}\right)\right| \mathrm{d} h \int_{Z \backslash A} \Phi_{X}\left(y^{-1} a h\right) \mathrm{d} a .
\end{aligned}
$$

Recall that $\phi\left(X^{h}\right) \eta(h)=\phi_{X}(h)=0$ unless $h \in C_{X}$. Suppose $h \in C_{X}$. We can assume $\log \|h\| \leq \log \left\|C_{X}\right\|$ and $\log \|y\|=\log \|y\|_{T \backslash H}$. Then $\Phi_{X}\left(y^{-1} a h\right)=0$ unless $y^{-1} a h \in \Omega_{X}$. Since

$$
1+\log \|a\|_{Z \backslash H} \leq(1+\log \|h\|)\left(1+\log \left\|y^{-1} a h\right\|_{Z \backslash H}\right)(1+\log \|y\|),
$$


we have $\Phi_{X}\left(y^{-1} a h\right)=0$ unless

$$
1+\log \|a\|_{Z \backslash H} \leq c_{2}\left(1+\log \left(\max \left\{1,\left|D^{\mathfrak{s}}(X)\right|_{F}^{-1}\right\}\right)\right)^{4},
$$

where $c_{2}=c_{1} c_{0}^{2}$. Therefore

$$
\begin{aligned}
\int_{Z \backslash A} \Phi_{X}\left(y^{-1} a h\right) \mathrm{d} a & \leq \int_{1+\log \|a\|_{Z \backslash H} \leq c_{2}\left(1+\log \left(\max \left\{1,\left|D^{\mathfrak{s}}(X)\right|_{F}^{-1}\right\}\right)\right)^{4}} \mathrm{~d} a \\
& \leq c_{3}\left(1+\log \left(\max \left\{1,\left|D^{\mathfrak{s}}(X)\right|_{F}^{-1}\right\}\right)\right)^{4 \ell}
\end{aligned}
$$

where $c_{3}$ is a positive constant, independent of the choice of $X \in \omega_{\mathfrak{c}}^{\prime}$, and $\ell=\operatorname{dim} Z \backslash A$. This shows that

$$
\left|\Theta_{t}\left(X^{y}\right)\right| \leq c_{3}\left(1+\log \left(\max \left\{1,\left|D^{\mathfrak{s}}(X)\right|_{F}^{-1}\right\}\right)\right)^{4 \ell} \int_{A \backslash H}\left|\phi\left(X^{h}\right)\right| \mathrm{d} h .
$$

Notice that Theorem 6.11 also holds when $\eta=\mathbf{1}$. Then we have:

$$
\sup _{X \in \omega_{\mathrm{c}}^{\prime}}\left|D^{\mathfrak{s}}(X)\right|_{F}^{\frac{1}{2}} \int_{A \backslash H}\left|\phi\left(X^{h}\right)\right| \mathrm{d} h<+\infty .
$$

Hence

$$
\left|\Theta_{t}\left(X^{y}\right)\right| \leq c_{4}\left|D^{\mathfrak{s}}(X)\right|_{F}^{-\frac{1}{2}}\left(1+\log \left(\max \left\{1,\left|D^{\mathfrak{s}}(X)\right|_{F}^{-1}\right\}\right)\right)^{4 \ell}
$$

for all $X \in \mathfrak{c}(F)$ and $y \in H$ such that $X^{h} \in \omega^{\prime}=\omega \cap \mathfrak{s}_{\mathrm{rs}}(F)$. Since there are only finitely many non-conjugate Cartan subspaces in $\mathfrak{s}$, there exists a constant $c_{5}$ such that

$$
\left|\Theta_{t}(X)\right| \leq c_{5}\left|D^{\mathfrak{s}}(X)\right|_{F}^{-\frac{1}{2}}\left(1+\log \left(\max \left\{1,\left|D^{\mathfrak{s}}(X)\right|_{F}^{-1}\right\}\right)\right)^{4 \ell}
$$

for all $X \in \omega^{\prime}$ and all $t \geq 1$.

It follows from the lemma below that the function

$$
X \mapsto\left|D^{\mathfrak{s}}(X)\right|_{F}^{-\frac{1}{2}}\left(1+\log \left(\max \left\{1,\left|D^{\mathfrak{s}}(X)\right|_{F}^{-1}\right\}\right)\right)^{4 \ell}
$$

is locally integrable on $\mathfrak{s}(F)$. Then Theorem 6.6 follows from Lebesgue's Theorem.

Lemma 6.20. There exists $\epsilon>0$ such that the function $\left|D^{\mathfrak{s}}(X)\right|_{F}^{-\epsilon}$ is locally integrable on $\mathfrak{c}(F)$ for any Cartan subspace $\mathfrak{c}$ of $\mathfrak{s}$.

Proof. See [Zhw1, Lemma 4.3].

\section{Local calculations}

\subsection{Limit formulae}

In this subsection, we obtain formulae for $\widehat{i}^{\eta}(X, Y)\left(X, Y \in \mathfrak{s}_{\mathrm{rs}}(F)\right)$ and $\widehat{i}(X, Y)$ $\left(X, Y \in \mathfrak{s}_{\mathrm{rs}}^{\prime}(F)\right)$ at "infinity", which are analogues of [Wa2, VIII.1 Proposition]. The proof of [Wa2, VIII.1 Proposition] is very technical. Here we modify Waldspurger's proof a little to make it available in our situation. 
Statement Let $\mathfrak{c}$ be a Cartan subspace of $\mathfrak{s}$, and $\mathbf{T}^{-}$the maximal $\theta$-split torus in $\mathbf{G}$ whose Lie algebra is $\mathfrak{c}$. Let $\mathbf{T}$ be the centralizer of $\mathfrak{c}$ in $\mathbf{H}$, and write $\mathfrak{t}=\operatorname{Lie}(\mathbf{T})$. For $X, Y \in \mathfrak{c}_{\text {reg }}(F)$, define a bilinear form $q_{X, Y}$ on $\mathfrak{h}(F) / \mathfrak{t}(F)$ by

$$
q_{X, Y}\left(Z, Z^{\prime}\right)=\left\langle[Z, X],\left[Y, Z^{\prime}\right]\right\rangle,
$$

where the pairing $\langle\cdot, \cdot\rangle$ is the one as before. One can check that the form $q_{X, Y}$ is nondegenerate and symmetric. One can also verify that $q_{X, Y}=q_{Y, X}$. We write $\gamma_{\psi}(X, Y)=\gamma_{\psi}\left(q_{X, Y}\right)$ for simplicity. Recall that, by conventions, $T=\mathbf{T}(F)$, $H=\mathbf{H}(F)$.

Let $\mathfrak{c}^{\prime}$ be a Cartan subspace of $\mathfrak{s}^{\prime}$. Similarly, we denote by $\mathbf{T}^{\prime-}$ the maximal $\theta$-split torus in $\mathbf{G}^{\prime}$ whose Lie algebra is $\mathfrak{c}^{\prime}$, by $\mathbf{T}^{\prime}$ the centralizer of $\mathfrak{c}^{\prime}$ in $\mathbf{H}^{\prime}$, and by $\mathfrak{t}^{\prime}$ the Lie algebra of $\mathbf{T}^{\prime}$. For $X, Y \in \mathfrak{c}_{\text {reg }}^{\prime}(F)$, we also define a nondegenerate, bilinear and symmetric form $q_{X, Y}$ on $\mathfrak{h}^{\prime}(F) / \mathfrak{t}^{\prime}(F)$ in the same way as above.

The following formulae depend on the choices of the Haar measures on $T$ and $H$ (also on $T^{\prime}$ and $H^{\prime}$ ). Here we equip $H$ or $T$ with the Haar measure so that the exponential map preserve the measure in a neighborhood of 0 in $\mathfrak{h}(F)$ or $\mathfrak{t}(F)$. We make the similar choices for the Haar measures on $T^{\prime}$ and $H^{\prime}$.

Proposition 7.1. Let the notations be as above.

1. Let $X \in \mathfrak{s}_{\mathrm{rs}}(F)$ and $Y \in \mathfrak{c}_{\mathrm{reg}}(F)$. Then there exists $N \in \mathbb{N}$ such that if $\mu \in F^{\times}$satisfying $v_{F}(\mu)<-N$, we have the equality

$$
\widehat{i}^{\eta}(\mu X, Y)=\kappa(Y) \sum_{h \in T \backslash H, h \cdot X \in \mathfrak{c}} \eta(h) \gamma_{\psi}(\mu h \cdot X, Y) \psi(\langle\mu h \cdot X, Y\rangle),
$$

and

$$
\widehat{i}^{\eta}(X, \mu Y)=\kappa(\mu Y) \sum_{h \in T \backslash H, h \cdot X \in \mathfrak{c}} \eta(h) \gamma_{\psi}(\mu h \cdot X, Y) \psi(\langle\mu h \cdot X, Y\rangle) .
$$

2. Let $X \in \mathfrak{s}_{\mathrm{rS}}^{\prime}(F)$ and $Y \in \mathfrak{c}_{\mathrm{reg}}^{\prime}(F)$. Then there exists $N \in \mathbb{N}$ such that if $\mu \in F^{\times}$satisfying $v_{F}(\mu)<-N$, we have the equality

$$
\widehat{i}(\mu X, Y)=\widehat{i}(X, \mu Y)=\sum_{h \in T^{\prime} \backslash H^{\prime}, h \cdot X \in \mathfrak{c}^{\prime}} \gamma_{\psi}(\mu h \cdot X, Y) \psi(\langle(\mu h \cdot X, Y\rangle) .
$$

In particular, the above expression is zero if $X$ is not conjugate to any element of $\mathfrak{c}(F)\left(\right.$ or $\left.\mathfrak{c}^{\prime}(F)\right)$.

Proof of Proposition 7.1 We now prove the formula for $\widehat{i}^{\eta}(\mu X, Y)$. The formula for $\widehat{i}^{\eta}(X, \mu Y)$ can be deduced from it. We leave the proof of the formulae for $\widehat{i}(\mu X, Y)$ and $\hat{i}(X, \mu Y)$ to the reader. They can be proved in the same way.

Firstly, we introduce some notations. Let $\mathfrak{q}$ (resp. $\mathfrak{p}$ ) be the unique complement of $\mathfrak{t}$ (resp. $\mathfrak{c}$ ) in $\mathfrak{h}$ (resp. $\mathfrak{s}$ ) which is stable under the adjoint action of $\mathbf{T}$. Denote by $S_{\mathfrak{c}}$ the set of roots of $\mathbf{T}^{-}$in $\mathfrak{g}(\bar{F})$. For each subspace $\mathfrak{f} \subset \mathfrak{g}(F)$ such that the restriction of $\langle\cdot, \cdot\rangle$ to $\mathfrak{f}$ is nondegenerate and for each $\mathcal{O}_{F}$-lattice $L \subset \mathfrak{f}$, set $\widetilde{L}=\left\{\ell \in \mathfrak{f}: \forall \ell^{\prime} \in L, \psi\left(\left\langle\ell^{\prime}, \ell\right\rangle\right)=1\right\}$. We denote by $L_{\mathfrak{c}}$ the $\mathcal{O}_{F}$-lattice of $\mathfrak{c}(F)$ such that

$$
\widetilde{L}_{\mathfrak{c}}=\left\{Z \in \mathfrak{c}(F): \forall \alpha \in S_{\mathfrak{c}}, v_{F}(\alpha(Z)) \geq 0\right\}
$$


Fix $\mathcal{O}_{F}$-lattices $L_{\mathfrak{p}} \subset \mathfrak{p}(F), L_{\mathfrak{t}} \subset \mathfrak{t}(F)$ and $L_{\mathfrak{q}} \subset \mathfrak{q}(F)$. Set $L_{\mathfrak{s}}=L_{\mathfrak{c}} \oplus L_{\mathfrak{p}}$, $L_{\mathfrak{h}}=L_{\mathfrak{t}} \oplus L_{\mathfrak{q}}, L=L_{\mathfrak{s}} \oplus L_{\mathfrak{h}}$.

For simplicity, write $d=\operatorname{dim}_{F}(\mathfrak{g}(F))=4 n^{2}$. Denote by $F[U]_{d}$ the set of monic polynomials of degree $d$ with coefficients in $F$. For $P \in F[U]_{d}$, write

$$
P(U)=\sum_{i=0}^{d} s_{i}(P) U^{d-i} .
$$

For $a \in \mathbb{Z}$ and $P_{1}, P_{2} \in F[U]_{d}$, we write $P_{1} \equiv P_{2} \bmod \varpi^{a} \mathcal{O}_{F}$ if $v_{F}\left(s_{i}\left(P_{1}\right)-\right.$ $\left.s_{i}\left(P_{2}\right)\right) \geq a$ for each $i=0,1, \ldots, d$. For each $Z \in \mathfrak{g}(F)$, denote by $P_{Z}$ the characteristic polynomial of $\operatorname{ad}(Z)$ acting on $\mathfrak{g}(F)$. Then $P_{Z} \in F[U]_{d}$.

Fix an integer $c \in \mathbb{N}$ satisfying the following conditions.

1. For each $a \in \mathbb{N}, a \geq c$, we have

- $\varpi^{a} L_{\mathfrak{h}} \subset V_{\mathfrak{h}}$ and $\varpi^{a} L \subset V_{\mathfrak{g}}$;

- $K_{a}:=\exp \left(\varpi^{a} L_{\mathfrak{h}}\right)$ is a subgroup of $K=\mathrm{GL}_{n}\left(\mathcal{O}_{F}\right) \times \mathrm{GL}_{n}\left(\mathcal{O}_{F}\right)$, and $\left.\eta\right|_{K_{a}}=1$

- the action of $K_{a}$ stabilizes $L_{\mathfrak{s}}$ (hence stabilizes $\widetilde{L}_{\mathfrak{s}}$ ).

2. For each $a \in \mathbb{N}, a \geq c$, and each $Z \in \varpi^{a} L_{\mathfrak{h}}$, we have

- $(\exp Z) \cdot Y-Y-[Z, Y] \in \varpi^{2 a-c} L_{\mathfrak{s}}$;

- $(\exp Z) \cdot Y-Y-[Z, Y]-\frac{1}{2}[Z,[Z, Y]] \in \varpi^{3 a-c} L_{\mathfrak{s}}$.

3. Denote by $C(X)$ the set of $X^{\prime} \in \mathfrak{c}(F)$ satisfying that there exists $h \in H$ such that $h \cdot X^{\prime}=X$, which is a finite set. We require that:

- if $a \in \mathbb{N}, a \geq c, X^{\prime}, X^{\prime \prime} \in C(X)$, and $\gamma \in K_{a}$ satisfying $\gamma \cdot X^{\prime}=X^{\prime \prime}$, then $X^{\prime}=X^{\prime \prime}$;

- for each $X^{\prime} \in C(X)$, denote by $\widetilde{L}_{\mathfrak{q}}^{X^{\prime}}$ the dual of $L_{\mathfrak{q}}$ in $\mathfrak{q}(F)$ with respect to the form $q_{X^{\prime}, Y}$; then require $\varpi^{c} \widetilde{L}_{\mathfrak{q}}^{X^{\prime}} \subset 2 \varpi^{-c} L_{\mathfrak{q}}$.

4. If $Z \in \mathfrak{p}(F)$ satisfying $[Y, Z] \in \widetilde{L}_{\mathfrak{h}}$, then $Z \in \varpi^{-c} \widetilde{L}_{\mathfrak{p}}$.

5. For each $h \in H$, denote by $c(h)$ the unique element of $\mathbb{Z}$ such that

$$
X^{h} \in \varpi^{-c(h)} \widetilde{L}_{\mathfrak{s}}-\varpi^{-c(h)+1} \widetilde{L}_{\mathfrak{s}} .
$$

Since $X \in \mathfrak{s}_{\mathrm{rs}}(F)$, the set $\{c(h), h \in H\}$ has a lower bound. We require that

- for each $h \in H, c(h) \geq-c$.

6. Fix a basis $\mathcal{B}$ of $\mathfrak{g}(\bar{F})$ formed of basis of $\mathfrak{c}(\bar{F})$ and $\mathfrak{t}(\bar{F})$, and root vectors associated to $S_{\mathfrak{c}}$. We require that

- for each $Z \in \widetilde{L}_{\mathfrak{p}}$, the coefficients of the matrix representation of $\operatorname{ad}(Z)$ with respect to the basis $\mathcal{B}$ are of valuation $\geq-c$;

- for each $i=0,1, \ldots, d, v_{F}\left(s_{i}\left(P_{X}\right)\right) \geq-c$. 
7. There exists an open compact set $\Omega \subset \mathfrak{c}_{\text {reg }}(F)$ such that if $Z \in \mathfrak{c}_{\text {reg }}(F)$ satisfying $P_{Z} \equiv P_{X} \bmod \varpi^{c} \mathcal{O}_{F}$, then $Z \in \Omega$.

The integer $c$ is fixed from now on. We also fix an open compact $\Omega$ satisfying condition (vii). The following lemma actually is [Wa2, VIII.3 Lemme], and whose proof can be applied in our situation.

Lemma 7.2. There exists $c^{\prime} \in \mathbb{N}, c^{\prime} \geq c$, such that if $a \in \mathbb{N}, a \geq c^{\prime}$, and $Z \in \Omega+\varpi^{a+c^{\prime}} \widetilde{L}_{\mathfrak{p}}$, then there exists $\gamma \in K_{a}$ such that $\gamma \cdot Z \in \Omega$.

From now on, we fix an integer $c^{\prime}$ as in the above lemma. Set

$$
N=2(d+8) c+6 c^{\prime}+12 .
$$

Let $\mu \in F^{\times}$be such that $v_{F}(\mu)<-N$. Choose $m \in \mathbb{N}$ such that

- the functions $Y^{\prime} \mapsto \widehat{i}^{\eta}\left(\mu X, Y^{\prime}\right), Y^{\prime} \mapsto\left|D^{\mathfrak{s}}\left(Y^{\prime}\right)\right|_{F}$ and $\kappa(Y)$ are constant on $Y+\varpi^{m} L_{\mathfrak{s}}$;

- for each $X^{\prime} \in C(X), \mu X^{\prime} \in \varpi^{-m} \widetilde{L}_{\mathfrak{s}}$.

Let $f$ be the characteristic function of $Y+\varpi^{m} L_{\mathfrak{s}}$, and $f^{\prime}$ be the characteristic function of $\varpi^{-m} \widetilde{L}_{\mathfrak{s}}$. Then we have

$$
\begin{aligned}
\widehat{I}^{\eta}(\mu X, f) & =\int_{\mathfrak{s}(F)} \widehat{i}^{\eta}\left(\mu X, Y^{\prime}\right) \kappa\left(Y^{\prime}\right) f\left(Y^{\prime}\right)\left|D^{\mathfrak{s}}\left(Y^{\prime}\right)\right|_{F}^{-1 / 2} \mathrm{~d} Y^{\prime} \\
& =\operatorname{vol}\left(\varpi^{m} L_{\mathfrak{s}}\right)\left|D^{\mathfrak{s}}(Y)\right|_{F}^{-1 / 2} \kappa(Y) \widehat{i}^{\eta}(\mu X, Y) .
\end{aligned}
$$

On the other hand, it is easy to verify that

$$
\widehat{f}\left(Y^{\prime}\right)=\operatorname{vol}\left(\varpi^{m} L_{\mathfrak{s}}\right) \psi\left(\left\langle Y, Y^{\prime}\right\rangle\right) f^{\prime}\left(Y^{\prime}\right) .
$$

Hence

$$
\widehat{I}^{\eta}(\mu X, f)=\left|D^{\mathfrak{s}}(\mu X)\right|_{F}^{1 / 2} \operatorname{vol}\left(\varpi^{m} L_{\mathfrak{s}}\right) \int_{T \backslash H} f^{\prime}\left(\mu X^{h}\right) \psi\left(\left\langle Y, \mu X^{h}\right\rangle\right) \eta(h) \mathrm{d} h .
$$

Set

$$
a=\left[-v_{F}(\mu) / 2\right]-2 c-c^{\prime}-1 .
$$

By (1), $a \geq c$. Fix a set of representatives $\Gamma$ in $H$ for the double coset $T \backslash H / K_{a}$. By condition (iii), we can suppose that if there exist $h \in \Gamma$ and $h^{\prime} \in T h K_{a}$ such that $X^{h^{\prime}} \in \mathfrak{c}(F)$, then $X^{h} \in \mathfrak{c}(F)$. Then we have

$$
\widehat{i}^{\eta}(\mu X, Y)=\left|D^{\mathfrak{s}}(\mu X) D^{\mathfrak{s}}(Y)\right|_{F}^{1 / 2} \kappa(Y) \sum_{h \in \Gamma} \operatorname{vol}\left(T \backslash T h K_{a}\right) f^{\prime}\left(\mu X^{h}\right) \eta(h) i(h),
$$

where

$$
i(h)=\int_{K_{a}} \psi\left(\left\langle Y, \mu X^{h \gamma}\right\rangle\right) \mathrm{d} \gamma .
$$

Fix $h \in \Gamma$. Choose $b \in \mathbb{N}$ such that

- $\left(c+c(h)-v_{F}(\mu)\right) / 2 \leq b \leq c(h)-v_{F}(\mu)-1-2 c ;$

- if $c(h) \leq c, b \leq\left\{\begin{array}{l}-(d+2) c-1-v_{F}(\mu), \\ -c-c^{\prime}-1-a-v_{F}(\mu),\end{array}\right.$ 
which implies $b \geq a$. Fix a set of representatives $\Gamma^{\prime}$ of $K_{a} / K_{b}$. Then we have

$$
i(h)=\sum_{g \in \Gamma^{\prime}} i(h, g),
$$

where

$$
i(h, g)=\int_{K_{b}} \psi\left(\left\langle Y, \mu X^{h g \gamma}\right\rangle\right) \mathrm{d} \gamma .
$$

Fix $g \in \Gamma^{\prime}$, and set $X^{\prime}=X^{h g}$. Then

$$
i(h, g)=\int_{\varpi^{b} L_{\mathfrak{h}}} \psi\left(\left\langle\exp Z \cdot Y, \mu X^{\prime}\right\rangle\right) \mathrm{d} Z .
$$

Notice that since $K_{b}$ stabilizes $L_{\mathfrak{s}}$ and $\widetilde{L}_{\mathfrak{s}}$, then $c(h g)=c(h)$. In particular, $X^{\prime} \in \varpi^{-c(h)} \widetilde{L}_{\mathfrak{s}}$. By (4), we have

$$
\psi\left(\left\langle Z, \mu X^{\prime}\right\rangle\right)=1
$$

for each $Z \in \varpi^{2 b-c} L_{\mathfrak{s}}$. Notice that $b \geq c$. For $Z \in \varpi^{b} L_{\mathfrak{h}}$, by condition (ii), we have

$$
\begin{aligned}
\psi\left(\left\langle\exp Z \cdot Y, \mu X^{\prime}\right\rangle\right) & =\psi\left(\left\langle Y+[Z, Y], \mu X^{\prime}\right\rangle\right) \\
& =\psi\left(\left\langle Y, \mu X^{\prime}\right\rangle\right) \psi\left(\left\langle Z,\left[Y, \mu X^{\prime}\right]\right\rangle\right) .
\end{aligned}
$$

Therefore we see that $i(h, g)=0$ if $\left[Y, \mu X^{\prime}\right] \notin \varpi^{-b} \widetilde{L}_{\mathfrak{h}}$. We make the following claim:

$$
\text { (*) } \quad \text { if }\left[Y, \mu X^{\prime}\right] \in \varpi^{-b} \widetilde{L}_{\mathfrak{h}} \text {, then } X^{h} \in \mathfrak{c}(F) .
$$

Now we prove this claim. Suppose $\left[Y, \mu X^{\prime}\right] \in \varpi^{-b} \widetilde{L}_{\mathfrak{h}}$, in other words, $\left[Y, X_{\mathfrak{p}}^{\prime}\right] \in$ $\mu^{-1} \varpi^{-b} \widetilde{L}_{\mathfrak{q}}$, where $X^{\prime}=X_{\mathfrak{c}}^{\prime}+X_{\mathfrak{p}}^{\prime}$ is the decomposition of $X^{\prime}$ with respect to $\mathfrak{s}=\mathfrak{c} \oplus \mathfrak{p}$. Thus, by condition (iv),

$$
X_{\mathfrak{p}}^{\prime} \in \mu^{-1} \varpi^{-b-c} \widetilde{L}_{\mathfrak{p}}
$$

Moreover, by $(4), X_{\mathfrak{p}}^{\prime} \in \varpi^{-c(h)+1} \widetilde{L}_{\mathfrak{p}}$. By the definition of $c(h)$ and that $c(h g)=$ $c(h)$, we deduce that

$$
X_{\mathfrak{c}}^{\prime} \in \varpi^{-c(h)} \widetilde{L}_{\mathfrak{c}}-\varpi^{-c(h)+1} \widetilde{L}_{\mathfrak{c}} .
$$

Set $R=\left\{\alpha \in S_{\mathfrak{c}}: v_{F}\left(\alpha\left(X_{\mathfrak{c}}^{\prime}\right)\right)<-c(h)+1\right\}$. The above relation and the definition of $\widetilde{L}_{\mathfrak{c}}$ imply that $R \neq \emptyset$. Set $r=\# R$, we calculate the coefficient $s_{r}\left(P_{X^{\prime}}\right)$. This is a sum of products of the coefficients of the matrix representations of $\operatorname{ad} X_{\mathfrak{c}}^{\prime}$ and $\operatorname{ad} X_{\mathfrak{p}}^{\prime}$ with respect to the basis $\mathcal{B}$. By (4), (5) and condition (vi), the coefficients of $\operatorname{ad} X_{\mathfrak{p}}^{\prime}$ are of valuation $\geq-c(h)+1$. The same relation holds for the coefficients of ad $X_{\mathfrak{c}}^{\prime}$ other than that of $\alpha\left(X_{\mathfrak{c}}^{\prime}\right)$ for $\alpha \in R$. The term $\prod_{\alpha \in R} \alpha\left(X_{\mathfrak{c}}^{\prime}\right)$, which occurs in $s_{r}\left(P_{X^{\prime}}\right)$, is of the valuation strictly less than that of any other term. Thus

$$
v_{F}\left(s_{r}\left(P_{X^{\prime}}\right)\right)=v_{F}\left(\prod_{\alpha \in R} \alpha\left(X_{\mathfrak{c}}^{\prime}\right)\right)<r(-c(h)+1) .
$$

Since $X^{\prime}$ is conjugate to $X$ by the action of $H$, then $P_{X^{\prime}}=P_{X}$. By condition (vi), we have

$$
-c<r(-c(h)+1)
$$


therefore

$$
c(h) \leq c .
$$

Let $i \in\{1,2, \ldots, d\}$. We now compare the coefficients $s_{i}\left(P_{X^{\prime}}\right)$ and $s_{i}\left(P_{X_{\mathrm{c}}^{\prime}}\right)$. Their difference is a sum of products of coefficients of the matrix representations of $\operatorname{ad} X_{\mathfrak{c}}^{\prime}$ and $\operatorname{ad} X_{\mathfrak{p}}^{\prime}$ with respect to the basis $\mathcal{B}$, and at least one coefficient of $\operatorname{ad} X_{\mathfrak{p}}^{\prime}$ is involved in these products. By (6), the coefficients of $\operatorname{ad} X_{c}^{\prime}$ are of valuation $\geq-c(h) \geq-c$. By (4), (5), (6) and condition (vi), the coefficients of ad $X_{\mathfrak{p}}^{\prime}$ are of valuation $\geq d c$. Therefore

$$
v_{F}\left(s_{i}\left(P_{X^{\prime}}\right)-s_{i}\left(P_{X_{\mathrm{c}}^{\prime}}\right)\right) \geq-(i-1) c+d c \geq c .
$$

In other words, $P_{X_{\mathfrak{c}}^{\prime}} \equiv P_{X^{\prime}} \bmod \varpi^{c} \mathcal{O}_{F}$. Thus, by condition (vii), $X_{\mathfrak{c}}^{\prime} \in \Omega$. By (4), (5) and (6), $X_{\mathfrak{p}}^{\prime} \in \varpi^{a+c^{\prime}} \widetilde{L}_{\mathfrak{p}}$. By (1) and (3), $a \geq c^{\prime}$. By Lemma 7.2, there exists $\gamma \in K_{a}$ such that $\gamma \cdot X^{\prime} \in \mathfrak{c}(F)$. By the choice of $\Gamma$, we have $X^{h} \in \mathfrak{c}(F)$. Now we have finished the proof the claim.

From now on, we suppose that $X^{h} \in \mathfrak{c}(F)$. Thus $f^{\prime}\left(\mu X^{h}\right)=1$ by the condition on $f^{\prime}$. Notice that the multiplication by $h^{-1}$ induces an isomorphism from $T \backslash T h K_{a}$ to $T \backslash T K_{a}$. Now we have

$$
\begin{aligned}
\widehat{i}^{\eta}(\mu X, Y)= & \kappa(Y)\left|D^{\mathfrak{s}}(\mu X) D^{\mathfrak{s}}(Y)\right|_{F}^{1 / 2} \operatorname{vol}\left(K_{a}\right)^{-1} \operatorname{vol}\left(T \backslash T K_{a}\right) \\
& \times \sum_{X^{\prime}=X^{h} \in C(X)} \eta(h) j\left(X^{\prime}\right)
\end{aligned}
$$

where

$$
\begin{aligned}
j\left(X^{\prime}\right) & =\int_{K_{a}} \psi\left(\left\langle Y, \mu X^{\prime \gamma}\right\rangle\right) \mathrm{d} \gamma \\
& =\int_{\varpi^{a} L_{\mathfrak{h}}} \psi\left(\left\langle\exp Z \cdot Y, \mu X^{\prime}\right\rangle\right) \mathrm{d} Z .
\end{aligned}
$$

Fix $X^{\prime} \in C(X)$. By (1) and (3), $\psi\left(\left\langle Y^{\prime}, \mu X^{\prime}\right\rangle\right)=1$ for $Y^{\prime} \in \varpi^{3 a-c} L_{\mathfrak{s}}$. Since $Y, X^{\prime} \in \mathfrak{c}(F)$, then for any $Z \in \mathfrak{g}(F),\left\langle[Z, Y], X^{\prime}\right\rangle=\left\langle Z,\left[Y, X^{\prime}\right]\right\rangle=0$. By condition (ii), we have

$$
\begin{aligned}
j\left(X^{\prime}\right) & =\psi\left(\left\langle Y, \mu X^{\prime}\right\rangle\right) \int_{\varpi^{a} L_{\mathfrak{h}}} \psi\left(\frac{1}{2}\left\langle[Z,[Z, Y]], \mu X^{\prime}\right\rangle\right) \mathrm{d} Z \\
& =\psi\left(\left\langle Y, \mu X^{\prime}\right\rangle\right) \int_{\varpi^{a} L_{\mathfrak{h}}} \psi\left(\frac{1}{2}\left\langle[Z, Y],\left[\mu X^{\prime}, Z\right]\right\rangle\right) \mathrm{d} Z \\
& =\psi\left(\left\langle Y, \mu X^{\prime}\right\rangle\right) \operatorname{vol}\left(\varpi^{a} L_{\mathfrak{t}}\right) \int_{\varpi^{a} L_{\mathfrak{q}}} \psi\left(\frac{1}{2} q_{\mu X^{\prime}, Y}(Z)\right) \mathrm{d} Z .
\end{aligned}
$$

Since $a \leq-c-v_{F}(\mu) / 2$ and by condition (iii), we obtain

$$
j\left(X^{\prime}\right)=\operatorname{vol}\left(\varpi^{a} L_{\mathfrak{t}}\right) \operatorname{vol}\left(\varpi^{a} L_{\mathfrak{q}}\right)^{1 / 2} \operatorname{vol}\left(\varpi^{-a} \breve{L}_{\mathfrak{q}}\right)^{1 / 2} \gamma_{\psi}\left(q_{\mu X^{\prime}, Y}\right) \psi\left(\left\langle Y, \mu X^{\prime}\right\rangle\right),
$$

where $\check{L}_{\mathfrak{q}}$ is the dual lattice of $L_{\mathfrak{q}}$ with respect to the form $q_{\mu X^{\prime}, Y}$. There is a relation:

$$
\operatorname{vol}\left(K_{a}\right)=\operatorname{vol}\left(T \backslash T K_{a}\right) \operatorname{vol}\left(T \cap K_{a}\right)=\operatorname{vol}\left(T \backslash T K_{a}\right) \operatorname{vol}\left(\varpi^{a} L_{\mathfrak{t}}\right) .
$$


By definition

$$
\begin{aligned}
\check{L}_{\mathfrak{q}} & =\left\{Z \in \mathfrak{q}(F): \forall Z^{\prime} \in L_{\mathfrak{q}}, \psi\left(\left\langle\left[Z, \mu X^{\prime}\right],\left[Y, Z^{\prime}\right]\right\rangle\right)=1\right\} \\
& \left.=\left\{Z \in \mathfrak{q}(F): \forall Z^{\prime} \in L_{\mathfrak{q}}, \quad \psi\left(\left\langle\left[\left[Z, \mu X^{\prime}\right], Y\right], Z^{\prime}\right]\right\rangle\right)=1\right\} \\
& =\left\{Z \in \mathfrak{q}(F):\left[\left[Z, \mu X^{\prime}\right], Y\right] \in \widetilde{L}_{\mathfrak{q}}\right\} .
\end{aligned}
$$

In other words,

$$
(\operatorname{ad} Y) \circ\left(\operatorname{ad} \mu X^{\prime}\right)\left(\check{L}_{\mathfrak{q}}\right)=\widetilde{L}_{\mathfrak{q}}
$$

and

$$
\operatorname{vol}\left(\check{L}_{\mathfrak{q}}\right)=\left|D^{\mathfrak{s}}(Y) D^{\mathfrak{s}}\left(\mu X^{\prime}\right)\right|_{F}^{-1} \operatorname{vol}\left(\widetilde{L}_{\mathfrak{q}}\right)
$$

On the other hand, we have the relation

$$
\operatorname{vol}\left(L_{\mathfrak{q}}\right) \operatorname{vol}\left(\widetilde{L}_{\mathfrak{q}}\right)=1
$$

Then Proposition 7.1 follows.

\subsection{Formulae for $\gamma_{\psi}(X, Y)$}

For $X, Y \in \mathfrak{c}_{\text {reg }}(F)$ or $\mathfrak{c}_{\text {reg }}^{\prime}(F)$, since $\gamma_{\psi}(X, Y)$ appears in the expression of $\widehat{i}^{\eta}(X, Y)$ or $\widehat{i}(X, Y)$ as in Proposition 7.1 , we need to know an explicit formula of $\gamma_{\psi}(X, Y)$. In this subsection, we show a formula (see Proposition 7.3) of $\gamma_{\psi}(X, Y)$ for $X, Y$ lying in a Cartan subspace of the Lie algebra associated to a general symmetric pair. This result is an analogue of [Wa2, VIII.5 Lemme].

Now we introduce some notations. Assume that $(\mathbf{G}, \mathbf{H}, \theta)$ is a general symmetric pair, as introduced in $\S 3$. Let $\mathfrak{s}$ be the Lie algebra associated to $(\mathbf{G}, \mathbf{H}, \theta)$, and $\mathfrak{c}$ a Cartan subspace of $\mathfrak{s}$. Let $\mathbf{T}$ be the centralizer of $\mathfrak{c}$ in $\mathbf{H}$ and write $\mathfrak{t}=\operatorname{Lie}(\mathbf{T})$. Fix a $\mathbf{G}$-invariant and $\theta$-invariant nondegenerate symmetric bilinear form $\langle$,$\rangle on \mathfrak{g}(F)$. Then, for $X, Y \in \mathfrak{c}_{\text {reg }}(F)$, the bilinear form $q_{X, Y}$ on $\mathfrak{h}(F) / \mathfrak{t}(F)$ defined by

$$
q_{X, Y}\left(Z, Z^{\prime}\right)=\left\langle[Z, X],\left[Y, Z^{\prime}\right]\right\rangle
$$

is nondegenerate and symmetric. Write $\gamma_{\psi}(X, Y)=\gamma_{\psi}\left(q_{X, Y}\right)$. For any subspace $\mathfrak{f}$ of $\mathfrak{g}(F)$ such that the restriction of $\langle$,$\rangle on \mathfrak{f}$ is nondegenerate, we write $\gamma_{\psi}(\mathfrak{f})$ for the Weil index associated to $\psi$ and the form $\langle$,$\rangle on \mathfrak{f}$.

Let $\mathbf{T}^{-}$be the maximal $\theta$-split torus in $\mathbf{G}$ whose Lie algebra is $\mathfrak{c}$. Denote by $S_{\mathfrak{c}}$ the set of roots of $\mathbf{T}^{-}$in $\mathfrak{g}(\bar{F})$. Write $\Gamma_{F}$ for the absolute Galois group $\operatorname{Gal}(\bar{F} / F)$. Then $\Gamma_{F}$ acts on $S_{\mathfrak{c}}$. For $\alpha \in S_{\mathfrak{c}}$, denote by $m_{\alpha}$ its multiplicity in $\mathfrak{g}(\bar{F})$. Since $\mathfrak{c} \subset \mathfrak{s}$, for $\alpha \in S_{\mathfrak{c}}$, we have $\theta(\alpha)=-\alpha$ and $m_{\alpha}=m_{-\alpha}$. For $\alpha \in S_{\mathfrak{c}}$, denote by $\Gamma_{ \pm \alpha}$ the stabilizer of $\{\alpha,-\alpha\}$ in $\Gamma_{F}$, by $F_{ \pm \alpha}$ the fixed field of $\Gamma_{ \pm \alpha}$ in $\bar{F}$, and by $S_{\mathfrak{c}}^{*}$ a fixed set of representatives of orbits $\{\alpha,-\alpha\}$. Notice that, if $X, Y \in \mathfrak{c}_{\text {reg }}(F), \alpha(X) \alpha(Y) \in F_{ \pm \alpha}$.

For $\alpha \in S_{\mathfrak{c}}^{*}$, denote by $\psi^{\prime}$ the character $\psi \circ \operatorname{Tr}_{F_{ \pm \alpha} / F}$ of $F_{ \pm \alpha}$. Set

$$
\gamma_{F_{ \pm \alpha}}\left(\alpha(X) \alpha(Y), \psi^{\prime}\right)=\frac{\gamma_{\psi^{\prime}}(\alpha(X) \alpha(Y) q)}{\gamma_{\psi^{\prime}}(q)}
$$

where $q$ is the quadratic form on $F_{ \pm \alpha}$ defined by $q(\lambda)=\lambda^{2}$. 
Proposition 7.3. Let the notations be as above. Then, for $X, Y \in \mathfrak{c}_{\mathrm{reg}}(F)$, we have

$$
\begin{aligned}
\gamma_{\psi}(X, Y)= & \gamma_{\psi}(\mathfrak{t}(F))^{-1} \gamma_{\psi}(\mathfrak{h}(F)) \\
& \times \prod_{\alpha \in S_{\mathfrak{c}}^{*}}\left((\alpha(X) \alpha(Y), 2)_{F_{ \pm \alpha}} \gamma_{F_{ \pm \alpha}}\left(\alpha(X) \alpha(Y), \psi^{\prime}\right)\right)^{m_{\alpha}} .
\end{aligned}
$$

where $(,)_{F_{ \pm \alpha}}$ is the Hilbert symbol on $F_{ \pm \alpha}$.

Proof. Notice that for $\alpha \in S_{\mathfrak{c}}$ we have $m_{\sigma \alpha}=m_{\alpha}$ for every $\sigma \in \Gamma_{F}$. For each root space $\mathfrak{g}_{\alpha}$ associated to $\alpha \in S_{\mathfrak{c}}$ we can choose its basis $\left\{E_{\alpha}^{1}, \ldots, E_{\alpha}^{m_{\alpha}}\right\}$ so that: (1) $\sigma\left(E_{\alpha}^{i}\right)=E_{\sigma \alpha}^{i}$ for each $\sigma \in \Gamma_{F}$; (2) $\theta\left(E_{\alpha}^{i}\right)=E_{-\alpha}^{i}$; (3) $\left\langle E_{\alpha}^{i}, E_{-\alpha}^{j}\right\rangle=\delta_{i j}$.

Consider the homomorphism

$$
\tau: \prod_{S_{c}^{*}} m_{\alpha} F_{ \pm \alpha} \longrightarrow \mathfrak{g}(\bar{F}), \quad\left(\lambda_{\alpha}^{i}\right) \mapsto \sum_{\alpha} \sum_{i=1}^{m_{\alpha}} \sum_{\sigma \in \Gamma / \Gamma_{ \pm \alpha}} \sigma\left(\lambda_{\alpha}^{i}\right)\left(E_{\sigma \alpha}^{i}+E_{-\sigma \alpha}^{i}\right) .
$$

In fact the image of $\tau$ lies in $\mathfrak{g}(F)$ and $\tau$ defines an isomorphism

$$
\prod_{S_{\mathfrak{c}}^{*}} m_{\alpha} F_{ \pm \alpha} \stackrel{\sim}{\longrightarrow} \mathfrak{q}(F),
$$

where $\mathfrak{q}$ is the unique complement of $\mathfrak{t}$ in $\mathfrak{h}$ which is stable under the adjoint action of $\mathbf{T}$. For $\left(\lambda_{\alpha}^{i}\right) \in \prod_{S_{c}^{*}} m_{\alpha} F_{ \pm \alpha}$, we have

$$
\begin{aligned}
q_{X, Y}\left(\tau\left(\left(\lambda_{\alpha}^{i}\right)\right)\right) & =\sum_{\alpha \in S_{c}^{*}} \sum_{i=1}^{m_{\alpha}} \sum_{\sigma \in \Gamma / \Gamma_{\alpha}} \sigma\left(\lambda_{\alpha}^{i}\right)^{2}\left\langle\left[E_{\sigma \alpha}^{i}+E_{-\sigma \alpha}^{i}, X\right],\left[Y, E_{\sigma \alpha}^{i}+E_{-\sigma \alpha}^{i}\right]\right\rangle \\
& =\sum_{\alpha, i, \sigma} \sigma\left(\lambda_{\alpha}^{i}\right)^{2}(-\sigma \alpha(X) \sigma \alpha(Y))\left\langle E_{\sigma \alpha}^{i}-E_{-\sigma \alpha}^{i}, E_{\sigma \alpha}^{i}-E_{-\sigma \alpha}^{i}\right\rangle \\
& =\sum_{\alpha, i, \sigma} \sigma\left(\lambda_{\alpha}^{i}\right)^{2} \sigma \alpha(X) \sigma \alpha(Y)\left\langle E_{\sigma \alpha}^{i}+E_{-\sigma \alpha}^{i}, E_{\sigma \alpha}^{i}+E_{-\sigma \alpha}^{i}\right\rangle \\
& =\sum_{\alpha \in S_{c}^{*}} \sum_{i=1}^{m_{\alpha}} q_{X, Y, \alpha}\left(\lambda_{\alpha}^{i}\right),
\end{aligned}
$$

where $q_{X, Y, \alpha}(\lambda)$ is the quadratic form on $F_{ \pm \alpha}$ defined by

$$
q_{X, Y, \alpha}(\lambda)=\operatorname{Tr}_{F_{ \pm \alpha} / F}\left(2 \alpha(X) \alpha(Y) \lambda^{2}\right) .
$$

Therefore

$$
\gamma_{\psi}(X, Y)=\prod_{\alpha \in S_{c}^{*}} \gamma_{\psi}\left(q_{X, Y, \alpha}\right)^{m_{\alpha}}
$$

For $\alpha \in S_{\mathfrak{c}}^{*}$, let $q_{X, Y, \alpha}^{\prime}$ be the quadratic form on $F_{ \pm \alpha}$ defined by:

$$
q_{X, Y, \alpha}^{\prime}(\lambda)=2 \alpha(X) \alpha(Y) \lambda^{2} .
$$

Then $\gamma_{\psi}\left(q_{X, Y, \alpha}\right)=\gamma_{\psi^{\prime}}\left(q_{X, Y, \alpha}^{\prime}\right)$, and

$$
\gamma_{\psi^{\prime}}\left(q_{X, Y, \alpha}^{\prime}\right)=(\alpha(X) \alpha(Y), 2)_{F_{ \pm \alpha}} \gamma_{F_{ \pm \alpha}}\left(\alpha(X) \alpha(Y), \psi^{\prime}\right) \gamma_{\psi^{\prime}}\left(q_{\alpha}^{\prime}\right),
$$


where $q_{\alpha}^{\prime}$ is the quadratic form on $F_{ \pm \alpha}$ defined by $q_{\alpha}^{\prime}(\lambda)=2 \lambda^{2}$. Therefore

$$
\gamma_{\psi}\left(q_{X, Y, \alpha}\right)=(\alpha(X) \alpha(Y), 2)_{F_{ \pm \alpha}} \gamma_{F_{ \pm \alpha}}\left(\alpha(X) \alpha(Y), \psi^{\prime}\right) \gamma_{\psi}\left(q_{\alpha}\right),
$$

where

$$
q_{\alpha}(\lambda)=\operatorname{Tr}_{F_{ \pm \alpha} / F}\left(2 \lambda^{2}\right)=\operatorname{Tr}_{F_{ \pm \alpha} / F}\left(\left\langle E_{\alpha}^{i}+E_{-\alpha}^{i}, E_{\alpha}^{i}+E_{-\alpha}^{i}\right\rangle \lambda^{2}\right) .
$$

In summary, we deduce that

$$
\gamma_{\psi}(X, Y)=\prod_{\alpha \in S_{c}^{*}}\left((\alpha(X) \alpha(Y), 2)_{F_{ \pm \alpha}} \gamma_{F_{ \pm \alpha}}\left(\alpha(X) \alpha(Y), \psi^{\prime}\right) \gamma_{\psi}\left(q_{\alpha}\right)\right)^{m_{\alpha}} .
$$

On the other hand, by the same argument as above, we can show that

$$
\gamma_{\psi}(\mathfrak{q}(F))=\prod_{\alpha \in S_{\mathfrak{c}}^{*}} \gamma_{\psi}\left(q_{\alpha}\right)^{m_{\alpha}} .
$$

Together with the obvious relation

$$
\gamma_{\psi}(\mathfrak{q}(F))=\gamma_{\psi}(\mathfrak{t}(F))^{-1} \gamma_{\psi}(\mathfrak{h}(F)),
$$

we complete the proof.

\subsection{Comparison lemma}

To obtain the main result of this subsection, we need the following lemma.

Lemma 7.4. Let $X \in \mathfrak{c}_{\text {reg }}(F)$ and $Y \in \mathfrak{c}_{\text {reg }}^{\prime}(F)$ be such that $X \leftrightarrow Y$. Then there exists an element $x \in \mathrm{GL}_{2 n}(E)$ such that $\operatorname{Ad}(x) Y=X$, and $\operatorname{Ad}(x)$ induces isomorphisms $\operatorname{Ad}(x): \mathfrak{t}^{\prime} \rightarrow \mathfrak{t}$ and $\operatorname{Ad}(x): \mathfrak{c}^{\prime} \rightarrow \mathfrak{c}$ over $F$.

Proof. It suffices to prove this for $X=\left(\begin{array}{cc}0 & \mathbf{1}_{n} \\ A & 0\end{array}\right)$ and $Y=\left(\begin{array}{cc}0 & \gamma B \\ \bar{B} & 0\end{array}\right)$, where $A \in \mathrm{GL}_{n}(F)$ is regular semisimple and $A=\gamma B \bar{B}$. Then we have

$$
\begin{aligned}
\mathfrak{c}(F) & =\left\{\left(\begin{array}{cc}
0 & C \\
A C & 0
\end{array}\right): C \in \mathfrak{g l}_{n}(F), A C=C A\right\}, \\
\mathfrak{t}(F) & =\left\{\left(\begin{array}{cc}
D & 0 \\
0 & D
\end{array}\right): D \in \mathfrak{g l}_{n}(F), A D=D A\right\}, \\
\mathfrak{c}^{\prime}(F) & =\left\{\left(\begin{array}{cc}
0 & \gamma P \\
\bar{P} & 0
\end{array}\right): P \in \mathfrak{g l}_{n}(E), B \bar{P}=P \bar{B}\right\},
\end{aligned}
$$

and

$$
\mathfrak{t}^{\prime}(F)=\left\{\left(\begin{array}{cc}
Q & 0 \\
0 & \bar{Q}
\end{array}\right): Q \in \mathfrak{g l}_{n}(E), B \bar{Q}=Q B\right\} .
$$

Take $x=\left(\begin{array}{cc}\mathbf{1}_{n} & 0 \\ 0 & \gamma B\end{array}\right) \in \mathrm{GL}_{2 n}(E)$. We claim that $\operatorname{Ad}(x)$ satisfies the required condition. By the above relation, it is easy to see that:

1. $\operatorname{Ad}(x) \cdot\left(\begin{array}{cc}0 & \gamma P \\ \bar{P} & 0\end{array}\right)=\left(\begin{array}{cc}0 & \gamma P B^{-1} \\ A P B^{-1} & 0\end{array}\right), A P B^{-1}=P B^{-1} A$ 
2. $\operatorname{Ad}(x) \cdot\left(\begin{array}{cc}Q & 0 \\ 0 & \bar{Q}\end{array}\right)=\left(\begin{array}{cc}Q & 0 \\ 0 & Q\end{array}\right), A Q=Q A$.

Therefore we have to show that $P B^{-1} \in \mathfrak{g l}_{n}(F), Q \in \mathfrak{g l}_{n}(F)$.

Note that since $A=B \bar{B}, A$ commutes with $B$. It is easy to see that $P$ and $Q$ also commute with $A$. Hence $P$ and $Q$ commute with $B$, since $A$ is regular. Therefore the relation $B \bar{P}=P \bar{B}$ implies that $P B^{-1}=\bar{P} \bar{B}^{-1}$; the relation $B \bar{Q}=Q B$ implies that $\bar{Q}=Q$, which concludes the proof.

Now let $X \in \mathfrak{c}_{\text {reg }}(F)$ and $Y \in \mathfrak{c}_{\text {reg }}^{\prime}(F)$ be such that $X \leftrightarrow Y$. Then we can take an $x \in \mathrm{GL}_{2 n}(E)$ as in the above lemma. For any $V \in \mathfrak{c}_{\text {reg }}^{\prime}(F)$, put $U=\operatorname{Ad}(x) V$.

Lemma 7.5. Let $X, Y, U, V$ be as above. Then we have the following relations

$$
\langle X, U\rangle=\langle Y, V\rangle
$$

and

$$
\gamma_{\psi}(X, U)=\gamma_{\psi}(\mathfrak{h}(F)) \gamma_{\psi}\left(\mathfrak{h}^{\prime}(F)\right)^{-1} \gamma_{\psi}(Y, V) .
$$

Proof. The first relation follows directly from the above lemma. The second relation follows from the above lemma, Proposition 7.1 and the similar arguments of equation (6) in [Wa1, page 96].

\subsection{Test functions}

This subsection is devoted to showing that we can construct specific $\mathcal{C}_{c}^{\infty}$-functions satisfying certain "good" matching conditions. Such functions will play an important role in proving Theorem 5.16 by global method. The result below is an analogue of [Wa3, Proposition in $\S 8.2]$.

Proposition 7.6. Let $Y_{0} \in \mathfrak{c}_{\text {reg }}^{\prime}(F) \subset \mathfrak{s}_{\text {rs }}^{\prime}(F)$ and $X_{0} \in \mathfrak{c}_{\text {reg }}(F) \subset \mathfrak{s}_{\mathrm{rs}}(F)$ be such that $X_{0} \leftrightarrow Y_{0}$. Then there exist functions $f \in \mathcal{C}_{c}^{\infty}(\mathfrak{s}(F))$ and $f^{\prime} \in \mathcal{C}_{c}^{\infty}\left(\mathfrak{s}^{\prime}(F)\right)$ satisfying the following conditions.

1. If $X \in \operatorname{Supp}(f)$, there exists $Y \in \mathfrak{c}_{\text {reg }}^{\prime}(F)$ such that $X \leftrightarrow Y$.

2. If $Y \in \operatorname{Supp}\left(f^{\prime}\right), Y$ is $H^{\prime}$-conjugate to an element in $\mathfrak{c}_{\mathrm{reg}}^{\prime}(F)$.

3. $f$ and $f^{\prime}$ are smooth transfer of each other.

4. There is an equality

$$
\begin{aligned}
& \qquad \kappa\left(X_{0}\right) \widehat{I}^{\eta}\left(X_{0}, f\right)=c \widehat{I}\left(Y_{0}, f^{\prime}\right) \neq 0, \\
& \text { where } c=\gamma_{\psi}(\mathfrak{h}(F)) \gamma_{\psi}\left(\mathfrak{h}^{\prime}(F)\right)^{-1} .
\end{aligned}
$$

Proof. Let $W_{\mathfrak{c}}$ (resp. $W_{\mathfrak{c}^{\prime}}$ ) be the Weyl group associated to $\mathfrak{c}$ (resp. $\left.\mathfrak{c}^{\prime}\right)$, i.e. $W_{\mathfrak{c}}=N_{H}(\mathfrak{c}) / Z_{H}(\mathfrak{c})\left(\operatorname{resp} . W_{\mathfrak{c}^{\prime}}=N_{H^{\prime}}\left(\mathfrak{c}^{\prime}\right) / Z_{H^{\prime}}\left(\mathfrak{c}^{\prime}\right)\right)$. Set

$$
C\left(X_{0}\right)=\left\{X \in \mathfrak{c}_{\text {reg }}(F): X=i\left(X_{0}\right) \text { for some } i \in W_{\mathfrak{c}}\right\},
$$

and

$$
C\left(Y_{0}\right)=\left\{Y \in \mathfrak{c}_{\mathrm{reg}}^{\prime}(F): Y=i\left(Y_{0}\right) \text { for some } i \in W_{\mathbf{c}^{\prime}}\right\} .
$$

By Lemma 7.4, we fix an isomorphism $\varphi: \mathfrak{c}^{\prime}(F) \rightarrow \mathfrak{c}(F)$ such that $\varphi\left(Y_{0}\right)=$ $X_{0}$. Fix $V_{0} \in \mathfrak{c}_{\text {reg }}^{\prime}(F)$ and $U_{0}:=\varphi\left(V_{0}\right) \in \mathfrak{c}_{\text {reg }}(F)$ so that if $X \in C\left(X_{0}\right)-X_{0}$ (resp. $\left.Y \in C\left(Y_{0}\right)-Y_{0}\right)$, we have $\left\langle X-X_{0}, U_{0}\right\rangle \neq 0$ (resp. $\left.\left\langle Y-Y_{0}, V_{0}\right\rangle \neq 0\right)$, and moreover, $\kappa\left(U_{0}\right)=\kappa\left(X_{0}\right)$. We make the following choices. 
1. Fix an integer $r \geq 1$ such that:

- $1+\varpi^{r} \mathcal{O}_{F} \subset F^{\times 2}$

- the sets $i\left(\left(1+\varpi^{r} \mathcal{O}_{F}\right) U_{0}\right)$ (resp. $\left.i\left(\left(1+\varpi^{r} \mathcal{O}_{F}\right) V_{0}\right)\right)$, for $i \in W_{\mathfrak{c}}$ (resp. $\left.i \in W_{\mathbf{c}^{\prime}}\right)$, are mutually disjoint.

2. There exists an integer $N$ such that if $\mu \in F^{\times}$satisfying $v_{F}(\mu)<-N$, we have

- for each $X \in C\left(X_{0}\right)-X_{0}$ (resp. $\left.Y \in C\left(Y_{0}\right)-Y_{0}\right)$, the character $\alpha \mapsto \psi\left(\varpi^{r} \mu \alpha\left\langle X-X_{0}, U_{0}\right\rangle\right)$ (resp. $\left.\alpha \mapsto \psi\left(\varpi^{r} \mu \alpha\left\langle Y-Y_{0}, Y_{0}\right\rangle\right)\right)$ is nontrivial on $\mathcal{O}_{F}$.

3. Fix $N$ and $\mu \in F^{\times}$with $v_{F}(\mu)<-N$ such that

- $\eta(\mu)=1$

- the condition (ii) above is satisfied;

- the formulae of Proposition 7.1 hold for $\widehat{i}^{\eta}\left(X_{0}, i\left(\mu U_{0}\right)\right)$ and $\widehat{i}\left(Y_{0}, i^{\prime}\left(\mu V_{0}\right)\right)$ for all $i \in W_{\mathbf{c}}$ and $i^{\prime} \in W_{\mathfrak{c}^{\prime}}$.

4. Set $\omega_{0}=\mu\left(1+\varpi^{r} \mathcal{O}_{F}\right) U_{0}$ and $\omega_{0}^{\prime}=\mu\left(1+\varpi^{r} \mathcal{O}_{F}\right) V_{0}$. Denote by $\mathfrak{d}$ (resp. $\left.\mathfrak{d}^{\prime}\right)$ the $F$-vector space generated by $U_{0}$ (resp. $\left.V_{0}\right)$, and fix a complement $\mathfrak{e}\left(\right.$ resp. $\left.\mathfrak{e}^{\prime}\right)$ in $\mathfrak{c}(F)\left(\right.$ resp. $\left.\mathfrak{c}^{\prime}(F)\right)$, i.e. $\mathfrak{c}(F)=\mathfrak{d} \oplus \mathfrak{e}\left(\right.$ resp. $\left.\mathfrak{c}^{\prime}(F)=\mathfrak{d}^{\prime} \oplus \mathfrak{e}^{\prime}\right)$. If $U \in \mathfrak{c}(F)$ (resp. $V \in \mathfrak{c}^{\prime}(F)$ ), denote by $U_{\mathfrak{d}}$ (resp. $\left.V_{\mathfrak{d}^{\prime}}\right)$ its projection on $\mathfrak{d}\left(\right.$ resp. $\left.\mathfrak{d}^{\prime}\right)$. We choose open compact neighborhoods $\omega_{\mathfrak{e}}$ and $\omega_{\mathfrak{e}^{\prime}}^{\prime}$ of 0 in $\mathfrak{e}$ and $\mathfrak{e}^{\prime}$ small enough so that: if we set $\omega=\omega_{0} \oplus \omega_{\mathfrak{e}}$ and $\omega^{\prime}=\omega_{0}^{\prime} \oplus \omega_{\mathfrak{e}^{\prime}}^{\prime}$, then

- the sets $i(\omega)\left(\right.$ resp. $\left.i^{\prime}\left(\omega^{\prime}\right)\right)$, for $i \in W_{\mathbf{c}}\left(\right.$ resp. $\left.i^{\prime} \in W_{\mathbf{c}^{\prime}}\right)$, are mutually disjoint;

- $\omega \subset \mathfrak{c}_{\text {reg }}(F), \omega^{\prime} \subset \mathfrak{c}_{\text {reg }}^{\prime}(F), \varphi\left(\omega^{\prime}\right)=\omega$, and therefore, for $U \in \omega, V \in$ $\omega^{\prime}$, they match with each other if and only if $\varphi(V)=U$;

- for each $X \in C\left(X_{0}\right)$ (resp. $Y \in C\left(Y_{0}\right)$ ), and each $U \in \omega$ (resp. $\left.V \in \omega^{\prime}\right)$,

$$
\widehat{i}^{\eta}(X, U)=\widehat{i}^{\eta}\left(X, U_{\mathfrak{d}}\right), \quad \widehat{i}(Y, V)=\widehat{i}\left(Y, V_{\mathfrak{d}^{\prime}}\right) ;
$$

- the function $\kappa$ is constant on $\omega$, which hence equals to $\kappa\left(X_{0}\right)$.

Define a function $f_{\omega}$ (resp. $\left.f_{\omega^{\prime}}^{\prime}\right)$ on $\omega\left(\right.$ resp. $\left.\omega^{\prime}\right)$ by

$$
\begin{aligned}
& f_{\omega}(U)=\psi\left(-\left\langle X_{0}, U_{\mathfrak{d}}\right\rangle\right), \quad \text { for } U \in \omega, \\
& f_{\omega^{\prime}}^{\prime}(V)=\psi\left(-\left\langle Y_{0}, V_{\mathfrak{d}^{\prime}}\right\rangle\right), \quad \text { for } V \in \omega^{\prime} .
\end{aligned}
$$

Now we fix a function $f \in \mathcal{C}_{c}^{\infty}(\mathfrak{s}(F))$ (resp. $\left.f^{\prime} \in \mathcal{C}_{c}^{\infty}\left(\mathfrak{s}^{\prime}(F)\right)\right)$ such that

$$
\begin{aligned}
& \operatorname{Supp}(f) \subset \omega^{H}, \quad \text { and } \kappa(U) I^{\eta}(U, f)=f_{\omega}(U) \quad \text { for each } U \in \omega, \\
& \operatorname{Supp}\left(f^{\prime}\right) \subset \omega^{\prime H^{\prime}} \text {, and } I\left(V, f^{\prime}\right)=f_{\omega^{\prime}}^{\prime}(V) \quad \text { for each } V \in \omega^{\prime} \text {. }
\end{aligned}
$$

Then, we have, for $X \in \mathfrak{s}_{\mathrm{rs}}(F)$,

$$
\kappa(X) I^{\eta}(X, f)= \begin{cases}f_{\omega}(U) & \text { if } X \text { is } H \text {-conjugate to some } U \in \omega, \\ 0 & \text { otherwise, }\end{cases}
$$


and for $Y \in \mathfrak{s}_{\mathrm{rs}}^{\prime}(F)$,

$$
I\left(Y, f^{\prime}\right)= \begin{cases}f_{\omega^{\prime}}^{\prime}(V) & \text { if } Y \text { is } H^{\prime} \text {-conjugate to some } V \in \omega^{\prime} \\ 0 & \text { otherwise. }\end{cases}
$$

Thus the assertions (i), (ii) and (iii) of the proposition follow from the above construction and Lemma 7.5.

To prove the assertion (iv), we observe that

$$
\begin{aligned}
\kappa\left(X_{0}\right) \widehat{I}^{\eta}\left(X_{0}, f\right) & =\kappa\left(X_{0}\right) \int_{\mathfrak{s}(F)} \widehat{i}^{\eta}\left(X_{0}, U\right) \kappa(U) f(U)\left|D^{\mathfrak{s}}(U)\right|^{-1 / 2} \mathrm{~d} U \\
& =\left|W_{\mathfrak{c}}\right|^{-1} \kappa\left(X_{0}\right) \int_{\mathfrak{c}(F)} \widehat{i}^{\eta}\left(X_{0}, U\right) \kappa(U) I^{\eta}(f, U) \mathrm{d} U \\
& =\kappa\left(X_{0}\right) \int_{\omega} \widehat{i}^{\eta}\left(X_{0}, U\right) f_{\omega}(U) \mathrm{d} U \\
& =\sum_{i \in W_{\mathfrak{c}}} \kappa\left(X_{0}\right) \int_{\omega} \eta(i) \kappa(U) \gamma_{\psi}\left(i\left(X_{0}\right), U\right) \psi\left(\left\langle i\left(X_{0}\right), U\right\rangle\right) f_{\omega}(U) \mathrm{d} U \\
& =\sum_{X \in C\left(X_{0}\right)} \operatorname{vol}\left(\omega_{\mathfrak{e}}\right) \kappa(X) \kappa(U) \int_{\omega_{0}} \gamma_{\psi}\left(X, U_{\mathfrak{o}}\right) \psi\left(\left\langle X-X_{0}, U_{\mathfrak{o}}\right\rangle\right) \mathrm{d} U_{\mathfrak{d}} \\
& =\sum_{X \in C\left(X_{0}\right)} \operatorname{vol}(\omega) \kappa(X) \kappa(U) \\
& \times \int_{\mathcal{O}_{F}} \gamma_{\psi}\left(X, \mu\left(1+\varpi^{r} \alpha\right) U_{0}\right) \psi\left(\left\langle X-X_{0}, \mu\left(1+\varpi^{r} \alpha\right) U_{0}\right\rangle\right) \mathrm{d} \alpha .
\end{aligned}
$$

By condition (i),

$$
\gamma_{\psi}\left(X, \mu\left(1+\varpi^{r} \alpha\right) U_{0}\right)=\gamma_{\psi}\left(X, \mu U_{0}\right), \quad \text { for any } \alpha \in \mathcal{O}_{F} .
$$

If $X \neq X_{0}$, by condition (ii),

$$
\int_{\omega_{0}} \psi\left(\left\langle X-X_{0}, \mu\left(1+\varpi^{r} \alpha\right) U_{0}\right\rangle\right) \mathrm{d} \alpha=0 .
$$

Therefore,

$$
\kappa\left(X_{0}\right) \widehat{I}^{\eta}\left(X_{0}, f\right)=\operatorname{vol}(\omega) \gamma_{\psi}\left(X_{0}, \mu U_{0}\right) \neq 0 .
$$

The same computation goes for $\widehat{I}\left(Y_{0}, f^{\prime}\right)$ and we get

$$
\widehat{I}\left(Y_{0}, f\right)=\operatorname{vol}\left(\omega^{\prime}\right) \gamma_{\psi}\left(Y_{0}, \mu V_{0}\right) \neq 0 .
$$

Then the conclusion follows from Lemma 7.5 and $\operatorname{vol}(\omega)=\operatorname{vol}\left(\omega^{\prime}\right)$.

\section{Proof of Theorem 5.16}

In this section, we will prove Theorem 5.16. We divide this theorem into two parts, i.e., Theorems 8.1 and 8.2 below.

Theorem 8.1. If $f$ is in $\mathcal{C}_{c}^{\infty}(\mathfrak{s}(F))_{0}$, so is $\widehat{f}$. 
Theorem 8.2. There exists a nonzero constant $c \in \mathbb{C}$ satisfying that: if $f \in$ $\mathcal{C}_{c}^{\infty}(\mathfrak{s}(F))$ and $f \in \mathcal{C}_{c}^{\infty}\left(\mathfrak{s}^{\prime}(F)\right)$ are smooth transfer of each other, then

$$
\kappa(X) \widehat{I}^{\eta}(X, f)=c \widehat{I}\left(Y, f^{\prime}\right)
$$

for any $X \in \mathfrak{s}_{\mathrm{rs}}(F)$ and $Y \in \mathfrak{s}_{\mathrm{rs}}^{\prime}(F)$ such that $X \leftrightarrow Y$.

We will use a local method to prove Theorem 8.1, and a global method to prove Theorem 8.2, as we have said before. The global method is a modification of that of [Wa3].

\subsection{Proof of Theorem 8.1}

By Lemma 5.15, it suffices to only consider the case $\mathfrak{s}^{\prime}=\mathfrak{s}_{\epsilon}^{\prime}$ when $\epsilon=1$. Throughout this subsection, we assume that $\epsilon=1$.

Recall that $\mathfrak{s}_{\mathrm{rs}}(F)_{0}$ is the subset of elements in $\mathfrak{s}_{\mathrm{rs}}(F)$ coming from $\mathfrak{s}_{\mathrm{rs}}^{\prime}(F)$. Let $\mathscr{C}_{0}$ be the set of Cartan subspaces of $\mathfrak{s}$ coming from those of $\mathfrak{s}^{\prime}$, and $\left|\mathscr{C}_{0}\right|$ a set of representatives for $\mathbf{H}$-conjugacy classes of Cartan subspaces in $\mathscr{C}_{0}$.

Let $f$ be in $\mathcal{C}_{c}^{\infty}(\mathfrak{s}(F))_{0}$. Then, by the Weyl integration formula, we have

$$
\begin{aligned}
\widehat{I}^{\eta}(X, f) & =\int_{\mathfrak{s}(F)} \widehat{i}^{\eta}(X, Y) \kappa(Y) f(Y)\left|D^{\mathfrak{s}}(Y)\right|^{-1 / 2} \mathrm{~d} Y \\
& =\sum_{\mathfrak{c}}\left|W_{\mathfrak{c}}\right|^{-1} \int_{\mathfrak{c}_{\mathrm{reg}}(F)} \widehat{i}^{\eta}(X, Y) \kappa(Y) I^{\eta}(Y, f) \mathrm{d} Y \\
& =\sum_{\mathfrak{c} \in\left|\mathscr{C}_{0}\right|}\left|W_{\mathfrak{c}}\right|^{-1} \int_{\mathfrak{c}_{\mathrm{reg}}(F)} \widehat{i}^{\eta}(X, Y) \kappa(Y) I^{\eta}(Y, f) \mathrm{d} Y .
\end{aligned}
$$

Thus, to show $\widehat{I^{\eta}}(X, f)=0$ for any $X \notin \mathfrak{s}_{\text {rs }}(F)_{0}$, it suffices to show the following lemma.

Lemma 8.3. For any $X \notin \mathfrak{s}_{\mathrm{rs}}(F)_{0}$ and any $Y \in \mathfrak{s}_{\mathrm{rs}}(F)_{0}$, we have $\widehat{i}^{\eta}(X, Y)=0$.

Proof. First we need some preparation. Define an involution $\tau$ on $\mathcal{C}_{c}^{\infty}(\mathfrak{s}(F))$ : $f^{\tau}(X):=f\left(X^{t}\right)$, where $X=\left(\begin{array}{cc}0 & A \\ B & 0\end{array}\right) \in \mathfrak{s}(F)$ and $X^{t}$ is its transpose. The following two properties can be easily checked:

1. $\tau$ commutes with Fourier transform, i.e., $(\widehat{f})^{\tau}=\widehat{f \tau}$;

2. for $X=\left(\begin{array}{cc}0 & A \\ B & 0\end{array}\right) \in \mathfrak{s}_{\mathrm{rs}}(F), I^{\eta}\left(X, f^{\tau}\right)=\eta(\operatorname{det} A B) I^{\eta}(X, f)$ for any $f \in$ $\mathcal{C}_{C}^{\infty}(\mathfrak{s}(F))$.

In particular, if $Y \in \mathfrak{s}_{\mathrm{rs}}(F)_{0}$, then $I^{\eta}\left(Y, f^{\tau}\right)=I^{\eta}(Y, f)$; if an elliptic $X$ is not in $\mathfrak{s}_{\mathrm{rs}}(F)_{0}$, then $I^{\eta}\left(X, f+f^{\tau}\right)=0$.

Now let $X \notin \mathfrak{s}_{\mathrm{rs}}(F)_{0}$ be an elliptic element. For any $f \in \mathcal{C}_{c}^{\infty}(\mathfrak{s}(F))_{0}$, by the above discussion, we see that

$$
0=\widehat{I}^{\eta}\left(X, f+f^{\tau}\right)=2 \sum_{\mathfrak{c} \in\left|\mathscr{C}_{0}\right|}\left|W_{\mathfrak{c}}\right|^{-1} \int_{\mathfrak{c}_{\mathrm{reg}}(F)} \widehat{i}^{\eta}(X, Y) \kappa(Y) I^{\eta}(Y, f) \mathrm{d} Y .
$$


For any $Y_{0} \in \mathfrak{s}_{\text {rs }}(F)_{0}$ we may choose a specific $f_{0} \in \mathcal{C}_{c}^{\infty}(\mathfrak{s}(F))_{0}$ so that

$$
\sum_{\mathfrak{c} \in\left|\mathscr{C}_{0}\right|}\left|W_{\mathfrak{c}}\right|^{-1} \int_{\mathfrak{c}_{\mathrm{reg}}(F)} \hat{i}^{\eta}(X, Y) \kappa(Y) I^{\eta}(Y, f) \mathrm{d} Y=\widehat{i}^{\eta}\left(X, Y_{0}\right) .
$$

Therefore $\widehat{i}^{\eta}(X, Y)=0$ for any elliptic $X \notin \mathfrak{s}_{\mathrm{rs}}(F)_{0}$ and any $Y \in \mathfrak{s}_{\mathrm{rs}}(F)_{0}$.

Now let $X \notin \mathfrak{s}_{\mathrm{rs}}(F)_{0}$ be a non-elliptic element. It suffices to assume that $X$ is of the form $X(A)=\left(\begin{array}{cc}0 & \mathbf{1}_{n} \\ A & 0\end{array}\right)$ for some $A \in \mathrm{GL}_{n, \mathrm{rs}}(F)$. Since $X \notin \mathfrak{s}_{\mathrm{rs}}(F)_{0}$ is non-elliptic, we can assume that $A$ is of the form $\left(\begin{array}{cc}A_{1} & 0 \\ 0 & A_{2}\end{array}\right)$ where $A_{1} \in$ $\mathrm{GL}_{n_{1}, \mathrm{rs}}(F)$ is elliptic and not in $\mathrm{N}\left(\mathrm{GL}_{n_{1}}(E)\right)$, and $A_{2}$ is in $\mathrm{GL}_{n_{2}, \mathrm{rs}}(F)$. Recall the discussions in $\S 6.1$. There is a subspace $\mathfrak{r} \simeq \mathfrak{s}_{n_{1}} \times \mathfrak{s}_{n_{2}}$ of $\mathfrak{s}$ such that $X \in \mathfrak{r}$. Moreover, under the natural isomorphism $\iota: \mathfrak{r} \stackrel{\sim}{\rightarrow} \mathfrak{s}_{n_{1}} \times \mathfrak{s}_{n_{2}}$, the image of $X$ is $\left(X_{1}, X_{2}\right)$ where $X_{i}=X\left(A_{i}\right)$ for $i=1,2$. Write $\mathfrak{s}_{i}=\mathfrak{s}_{n_{i}}$ for $i=1,2$. Let $M=H_{1} \times H_{2}$ where $H_{i}=H_{n_{i}}$ for $i=1,2$. Then $M$ acts on $\mathfrak{r}$ naturally. For $Z \in \mathfrak{r}_{\mathrm{rs}}(F)$, let $\hat{i}^{\eta, \mathfrak{r}}(Z, \cdot)$ be the kernel function that represents the distribution $f \mapsto \widehat{I}^{\eta, M}(Z, f)$ for $f \in \mathcal{C}_{c}^{\infty}(\mathfrak{r}(F))$. It is obvious that

$$
\widehat{i}^{\eta, \mathfrak{r}}(Z, Y)=\widehat{i}^{\eta, \mathfrak{s}_{1}}\left(Z_{1}, Y_{1}\right) \widehat{i}^{\eta, \mathfrak{s}_{2}}\left(Z_{2}, Y_{2}\right)
$$

where $\left(Z_{1}, Z_{2}\right)$ and $\left(Y_{1}, Y_{2}\right)$ are the images of $Z$ and $Y$ under $\iota$ in $\mathfrak{s}_{1} \times \mathfrak{s}_{2}$ respectively, and $\widehat{i}^{\eta, \mathfrak{s}_{i}}\left(Z_{i}, \cdot\right)$ is the kernel function that represents the distribution $f \mapsto \widehat{I}^{\eta, H_{i}}\left(Z_{i}, f\right)$ for $f \in \mathcal{C}_{c}^{\infty}\left(\mathfrak{s}_{i}(F)\right)$ for $i=1,2$.

By Proposition 6.3, we have

$$
\widehat{i}^{\eta}(X, Y)=\sum_{Y^{\prime}} \hat{i}^{\eta, \mathfrak{r}}\left(X, Y^{\prime}\right),
$$

where $Y^{\prime}$ runs over a set of representatives for the finitely many $M$-conjugacy classes of elements of $\mathfrak{r}(F)$ which are $H$-conjugate to $Y$. Therefore we can and do assume that $Y \in \mathfrak{s}_{\mathrm{rs}}(F)_{0}$ is in $\mathfrak{r}(F)$ and of the form $Y=\left(Y_{1}, Y_{2}\right)$ under the natural map $\iota$ where $Y_{i} \in \mathfrak{s}_{i \text {,rs }}(F)_{0}$. Then

$$
\widehat{i}^{\iota, \mathfrak{r}}(X, Y)=\widehat{i}^{\eta, \mathfrak{s}_{1}}\left(X_{1}, Y_{1}\right) \widehat{i}^{\eta, \mathfrak{s}_{2}}\left(X_{2}, Y_{2}\right)=0,
$$

since $X_{1} \notin \mathfrak{s}_{1, \mathrm{rs}}(F)_{0}$ is elliptic and $Y_{1} \in \mathfrak{s}_{1, \mathrm{rs}}(F)_{0}$. We complete the proof of the lemma.

\subsection{A result on convergence}

Now let $k$ be a number field, $\mathbb{A}=\mathbb{A}_{\infty} \times \mathbb{A}_{\mathrm{f}}$ its ring of adeles. Let $k^{\prime}$ be a quadratic field extension of $k, \mathbb{D}$ a quaternion algebra over $k$ containing $k^{\prime}$, and $\eta$ the quadratic character of $\mathbb{A}^{\times} / k^{\times}$attached to $k^{\prime}$ by the class field theory. We define the global symmetric pairs $(\mathbf{G}, \mathbf{H})$ and $\left(\mathbf{G}^{\prime}, \mathbf{H}^{\prime}\right)$ over $k$ with respect to $k^{\prime}$ and $\mathbb{D}$ similarly as the local cases. Let $\mathfrak{s}, \mathfrak{s}^{\prime}$ be the corresponding global "Lie algebras" associated to $(\mathbf{G}, \mathbf{H})$ and $\left(\mathbf{G}^{\prime}, \mathbf{H}^{\prime}\right)$ respectively, which are defined over $k$. Denote by $\mathcal{S}(\mathfrak{s}(\mathbb{A}))$ (resp. $\mathcal{S}\left(\mathfrak{s}^{\prime}(\mathbb{A})\right)$ ) the space of Schwartz functions on $\mathfrak{s}(\mathbb{A})\left(\right.$ resp. $\left.\mathfrak{s}^{\prime}(\mathbb{A})\right)$. Denote by $\mathbf{H}(\mathbb{A})^{1}$ the set of $\left(h_{1}, h_{2}\right) \in \mathbf{H}(\mathbb{A})$ such that $\left|\operatorname{det} h_{1}\right|=\left|\operatorname{det} h_{2}\right|=1$, and by $\mathbf{H}^{\prime}(\mathbb{A})^{1}$ the set of $h \in \mathbf{H}^{\prime}(\mathbb{A})$ such that $|\operatorname{det} h|=1$. The groups $\mathbf{H}(\mathbb{A})^{1}$ and $\mathbf{H}^{\prime}(\mathbb{A})^{1}$ are subgroups of $\mathbf{H}(\mathbb{A})$ and $\mathbf{H}^{\prime}(\mathbb{A})$ respectively. We have the following theorem concerning the issue about convergence. 
Theorem 8.4. For each $\phi \in \mathcal{S}(\mathfrak{s}(\mathbb{A}))$,

$$
\int_{\mathbf{H}(k) \backslash \mathbf{H}(\mathbb{A})^{1}} \sum_{X \in \mathfrak{S}_{\text {ell }}(k)}\left|\phi\left(X^{h}\right)\right| \mathrm{d} h<\infty .
$$

Similarly, for each $\phi^{\prime} \in \mathcal{S}\left(\mathfrak{s}^{\prime}(\mathbb{A})\right)$,

$$
\int_{\mathbf{H}^{\prime}(k) \backslash \mathbf{H}^{\prime}(\mathbb{A})^{1}} \sum_{Y \in \mathfrak{s}_{\text {ell }}^{\prime}(k)}\left|\phi^{\prime}\left(Y^{h}\right)\right| \mathrm{d} h<\infty .
$$

Proof. (1) Now we prove the assertion for $\phi \in \mathcal{S}(\mathfrak{s}(\mathbb{A}))$. Here we still write $Z=(X, Y) \in \mathfrak{s}=\mathfrak{g l}_{n} \oplus \mathfrak{g l}_{n}$ and $h \cdot Z=(\operatorname{Ad} h) Z$ where $h \in \mathbf{H}$ for convenience. Recall that $Z=(X, Y)$ is in $\mathfrak{s}_{\text {ell }}(k)$ if and only if neither $X Y$ nor $Y X$ is contained in a proper parabolic subgroup of $\mathrm{GL}_{n}(k)$. Let $\mathbf{P}_{0}$ be the minimal parabolic subgroup of $\mathrm{GL}_{n}$ consisting of the upper-triangular one. Put $\mathbf{P}=\mathbf{P}_{0} \times \mathbf{P}_{0} \subset \mathbf{H}$. Identify $\mathbb{R}_{+}^{\times}$with the subgroup of $\mathbb{A}_{\infty}^{\times}$consisting of elements whose components at each place are the same and belong to $\mathbb{R}_{+}^{\times}$. For each real number $c>0$, put $A_{c}^{0}$ the set of $a=\operatorname{diag}\left(a_{1}, \ldots, a_{n}\right) \in \mathrm{SL}_{n}(\mathbb{R})$ such that $\frac{a_{i}}{a_{i+1}} \geq c$ for all $1 \leq i \leq n-1$ and $a_{i} \in \mathbb{R}_{+}^{\times}$for all $1 \leq i \leq n$, and set

$$
A_{c}=A_{c}^{0} \times A_{c}^{0} \subset \mathbf{H}(\mathbb{R}) \subset \mathbf{H}\left(\mathbb{A}_{\infty}\right) .
$$

By reduction theory, we know that there exists a maximal compact subgroup $\mathbf{K}$ of $\mathbf{H}(\mathbb{A})$, a compact subset $\omega \subset \mathrm{P}(\mathbb{A}) \cap \mathbf{H}(\mathbb{A})^{1}$ and a $c>0$ such that, if we set

$$
\mathcal{G}=\left\{p a k ; p \in \omega, a \in A_{c}, k \in \mathbf{K}\right\},
$$

we have the equality $\mathbf{H}(\mathbb{A})^{1}=\mathbf{H}(k) \mathcal{G}$, and thus, for each measurable function $\phi$ on $\mathbf{H}(k) \backslash \mathbf{H}(\mathbb{A})^{1}$ with valued $\geq 0$, the integral

$$
\int_{\mathbf{H}(k) \backslash \mathbf{H}(\mathbb{A})^{1}} \phi(x) \mathrm{d} x
$$

is convergent if and only if the integral

$$
\int_{\mathcal{G}} \phi(x) \mathrm{d} x
$$

is such so. Fix such $\mathbf{K}, \omega, c$. Then the integral is convergent if there exists $C \geq 0$ such that for each $p \in \omega, k \in \mathbf{K}$,

$$
\int_{A_{c}} \sum_{Z \in \mathfrak{s}_{\mathrm{ell}}(k)}|\phi((p a k) \cdot Z)| \delta_{\mathbf{P}}(a)^{-1} \mathrm{~d} a \leq C,
$$

where $\delta_{\mathbf{P}}$ is the modulus character of $\mathbf{P}$. There exists a compact set $\Omega \subset \mathbf{H}(\mathbb{A})^{1}$ such that for all $p \in \omega, a \in A_{c}, k \in \mathbf{K}, a^{-1} p a k \in \Omega$. Then there exists $\phi^{\prime} \in$ $\mathcal{S}(\mathfrak{s}(\mathbb{A}))$ such that for all $Z \in \mathfrak{s}(\mathbb{A})$ and $h \in \Omega$, we have $|\phi(h \cdot Z)| \leq \phi^{\prime}(Z)$. It suffices to consider $\phi^{\prime}$ of the form $\phi^{\prime}=\phi_{\infty}^{\prime} \otimes \phi_{\mathrm{f}}^{\prime}$, where $\phi_{\infty}^{\prime} \in \mathcal{S}\left(\mathfrak{s}\left(\mathbb{A}_{\infty}\right)\right), \phi_{\mathrm{f}}^{\prime} \in$ $\mathcal{S}\left(\mathfrak{s}\left(\mathbb{A}_{\mathrm{f}}\right)\right)$ both with $\geq 0$ valued, and suffices to consider the integral

$$
\int_{A_{c}} \sum_{Z \in \mathfrak{s}_{\text {ell }}(k)} \phi^{\prime}(a \cdot Z) \delta_{\mathbf{P}}(a)^{-1} \mathrm{~d} a .
$$


Choose an $\mathcal{O}_{k}$-lattice $L$ in $\mathfrak{s}(k)$ such that $\mathfrak{s}(k) \cap \operatorname{Supp}\left(\phi_{\mathrm{f}}\right) \subset L$. Denote $L_{\text {ell }}=$ $L \cap \mathfrak{s}_{\text {ell }}(k)$. Since $\phi_{\mathrm{f}}^{\prime}(a \cdot Z)=\phi_{\mathrm{f}}^{\prime}(Z)$, it suffices to consider the integral

$$
\int_{A_{c}} \sum_{Z \in L_{\mathrm{ell}}(k)} \phi_{\infty}^{\prime}(a \cdot Z) \delta_{\mathbf{P}}(a)^{-1} \mathrm{~d} a .
$$

If $x_{v} \in k_{v}$ and $v$ is an infinite place of $k$, write $\left|x_{v}\right|$ for the usual absolute value of $x_{v}$. For every $x=\left(x_{v}\right) \in \mathbb{A}_{\infty}$, put $|x|=\max _{v}\left|x_{v}\right|$. For $X=\left(x_{i, j}\right) \in \mathfrak{g l}_{n}\left(\mathbb{A}_{\infty}\right)$, put $|X|=\max _{i, j}\left|x_{i, j}\right|$. For $Z=(X, Y) \in \mathfrak{s}\left(\mathbb{A}_{\infty}\right)$, write $|Z|=\max \{|X|,|Y|\}$. Then the following lemma implies the theorem.

Lemma 8.5. Assume that $n \geq 2$. There is a positive valued polynomial function $P$ on the real vector space $\mathfrak{s}\left(\mathbb{A}_{\infty}\right)$, which depends on $L$ and $c$, such that

$$
P(a \cdot Z) \geq\left(\prod_{i=1}^{n-1} \frac{a_{i}}{a_{i+1}} \cdot \frac{b_{i}}{b_{i+1}}\right)|Z|,
$$

for all $a=\operatorname{diag}\left(a_{1}, \ldots, a_{n}, b_{1}, \ldots, b_{n}\right) \in A_{c}$ and all $Z \in L_{\mathrm{ell}}$.

Proof. Take a positive valued polynomial function $P_{1}$ on $\mathfrak{s}\left(\mathbb{A}_{\infty}\right)$ such that

$$
P_{1}(X, Y) \geq \max \{|X Y|,|Y X|\}, \quad \text { for all }(X, Y) \in \mathfrak{s}\left(\mathbb{A}_{\infty}\right) .
$$

Take a positive number $c_{L}$ such that

$$
(X, Y) \in L, d \text { is a nonzero entry of } X Y \text { or } Y X \Rightarrow|d| \geq c_{L} .
$$

Let $a=\left(a_{1}, a_{2}, \cdots, a_{n}, b_{1}, b_{2}, \cdots, b_{n}\right)$ in $A_{c}$ and let $Z=(X, Y)=\left(\left(x_{i, j}\right),\left(y_{i, j}\right)\right)$ in $L_{\text {ell }}$. Write $\left(u_{i, j}\right)$ for $X Y$. Fix $i_{0}=1,2, \cdots, n-1$. Since $X Y$ is not contained in a proper parabolic subalgebra of $\mathfrak{g l}_{n}(k)$, there are $i \geq i_{0}+1$ and $j \leq i_{0}$ such that

$$
u_{i, j} \neq 0 \text {. }
$$

Then

$$
\left|u_{i, j}\right| \geq c_{L}
$$

and we have

$$
\begin{aligned}
P_{1}(a \cdot Z) & \geq\left|a_{i} u_{i, j} a_{j}^{-1}\right| \geq c_{L} a_{i} a_{j}^{-1} \geq c_{L} c^{i-i_{0}-1} c^{i_{0}-j} a_{i_{0}} a_{i_{0}+1}^{-1} \\
& \geq c_{L} c^{n-2} a_{i_{0}} a_{i_{0}+1}^{-1} .
\end{aligned}
$$

This implies that

$$
a_{n}^{-1}=\prod_{i=1}^{n-1}\left(a_{i} / a_{i+1}\right)^{\frac{i}{n}} \leq\left(\frac{1}{c_{L} c^{n-2}} P_{1}(a \cdot Z)\right)^{\frac{n-1}{2}},
$$

and

$$
a_{1}=\prod_{i=1}^{n-1}\left(a_{i} / a_{i+1}\right)^{\frac{n-i}{n}} \leq\left(\frac{1}{c_{L} c^{n-2}} P_{1}(a \cdot Z)\right)^{\frac{n-1}{2}} .
$$

Similarly,

$$
P_{1}(a \cdot Z) \geq c_{L} c^{n-2} b_{i_{0}} b_{i_{0}+1}^{-1},
$$


and

$$
b_{1}, b_{n}^{-1} \leq\left(\frac{1}{c_{L} c^{n-2}} P_{1}(a \cdot Z)\right)^{\frac{n-1}{2}} .
$$

For all $i, j=1,2, \cdots, n$, we have

$$
|a \cdot Z| \geq a_{i}\left|a_{i, j}\right| b_{j}^{-1} .
$$

Therefore,

$$
\begin{aligned}
\left|a_{i, j}\right| & \leq a_{i}^{-1} b_{j}|a \cdot Z| \leq c^{-(n-i)-(j-1)} a_{n}^{-1} b_{1}|a \cdot Z| \\
& \leq c^{-(2 n-2)}\left(\frac{1}{c_{L} c^{n-2}}\right)^{n-1} P_{1}(a \cdot Z)^{n-1}|a \cdot Z| .
\end{aligned}
$$

Similarly,

$$
\left|b_{i, j}\right| \leq c^{-(2 n-2)}\left(\frac{1}{c_{L} c^{n-2}}\right)^{n-1} P_{1}(a \cdot Z)^{n-1}|a \cdot Z| .
$$

By timing $\frac{a_{i}}{a_{i+1}}$ and $\frac{b_{i}}{b_{i+1}}$ on both sides of the above inequality, we get the lemma.

(2) The convergence of the second integral (for $\phi^{\prime} \in \mathcal{S}^{\prime}(\mathfrak{s}(\mathbb{A}))$ ) can be deduced easily from Lemma 10.8 of [Wa3], since the twisted conjugation by $A_{c}$ is the usual conjugation. by

By the above theorem, we have a well-defined distribution $I^{\eta}$ on $\mathfrak{s}(\mathbb{A})$, defined

$$
I^{\eta}(\phi)=\int_{\mathbf{H}(k) \backslash \mathbf{H}(\mathbb{A})^{1}} \sum_{X \in \mathfrak{s}_{\text {ell }}(k)} \phi\left(X^{h}\right) \eta(h) \mathrm{d} h, \quad \phi \in \mathcal{S}(\mathfrak{s}(\mathbb{A})),
$$

and a well-defined distribution $I$ on $\mathfrak{s}^{\prime}(\mathbb{A})$, defined by

$$
I\left(\phi^{\prime}\right)=\int_{\mathbf{H}^{\prime}(k) \backslash \mathbf{H}^{\prime}(\mathbb{A})^{1}} \sum_{Y \in \mathfrak{s}_{\text {ell }}^{\prime}(k)} \phi^{\prime}\left(Y^{h}\right) \mathrm{d} h, \quad \phi^{\prime} \in \mathcal{S}\left(\mathfrak{s}^{\prime}(\mathbb{A})\right) .
$$

If $\phi=\prod_{v} \phi_{v}, \phi^{\prime}=\prod_{v} \phi_{v}^{\prime}$, it is routine to see that

$$
\begin{gathered}
I^{\eta}(\phi)=\sum_{X \in\left[\mathfrak{s}_{\text {ell }}(k)\right]} \tau\left(\mathbf{H}_{X}\right) \prod_{v} \kappa_{v}(X) I^{\eta}\left(X, \phi_{v}\right), \\
I\left(\phi^{\prime}\right)=\sum_{Y \in\left[\mathfrak{s}_{\text {ell }}(k)\right]} \tau\left(\mathbf{H}_{Y}^{\prime}\right) \prod_{v} I\left(Y, \phi_{v}^{\prime}\right),
\end{gathered}
$$

where

$$
\tau\left(\mathbf{H}_{X}\right)=\operatorname{vol}\left(\mathbf{H}_{X}(k) \backslash\left(\mathbf{H}_{X} \cap \mathbf{H}(\mathbb{A})^{1}\right)\right), \quad \tau\left(\mathbf{H}_{Y}^{\prime}\right)=\operatorname{vol}\left(\mathbf{H}_{Y}^{\prime}(k) \backslash\left(\mathbf{H}_{Y}^{\prime} \cap \mathbf{H}^{\prime}(\mathbb{A})^{1}\right),\right.
$$

$\left[\mathfrak{s}_{\text {ell }}(k)\right]$ denotes the set of $\mathbf{H}(k)$-orbits in $\mathfrak{s}_{\text {ell }}(k)$, and $\left[\mathfrak{s}_{\text {ell }}^{\prime}(k)\right]$ denotes the set of $\mathbf{H}^{\prime}(k)$-orbits in $\mathfrak{s}_{\text {ell }}^{\prime}(k)$. If $X \in \mathfrak{s}_{\mathrm{rs}}(k)$ and $Y \in \mathfrak{s}_{\mathrm{rs}}^{\prime}(k)$ so that $X \leftrightarrow Y$, then $\mathbf{H}_{X} \simeq \mathbf{H}_{Y}^{\prime}$ (same reason as the local case). We choose Haar measures on $\mathbf{H}_{X}(\mathbb{A})$ and $\mathbf{H}_{Y}^{\prime}(\mathbb{A})$ so that they are compatible. Thus, if $X \in \mathfrak{s}_{\text {ell }}(k), Y \in \mathfrak{s}_{\text {ell }}^{\prime}(k)$ such that $X \leftrightarrow Y$, we have

$$
\tau\left(\mathbf{H}_{X}\right)=\tau\left(\mathbf{H}_{Y}^{\prime}\right) .
$$




\subsection{Proof of Theorem 8.2}

Now, we fix $f \in \mathcal{C}_{c}^{\infty}(\mathfrak{s}(F))$ and $f^{\prime} \in \mathcal{C}_{c}^{\infty}\left(\mathfrak{s}^{\prime}(F)\right)$ so that they are smooth transfer of each other. Here we allow that $f$ may not lie in $\mathcal{C}_{c}^{\infty}(\mathfrak{s}(F))_{0}$, as we have mentioned in the proof of Proposition 5.17. We also refer the reader to the proof of Proposition 5.17 to see the definition of smooth transfer in this more general situation.

Fix $X_{0} \in \mathfrak{s}_{\mathrm{rs}}(F), Y_{0} \in \mathfrak{s}_{\mathrm{rs}}^{\prime}(F)$ such that $X_{0} \leftrightarrow Y_{0}$. Our aim is to search for a nonzero constant $c$ which is independent of $f, f^{\prime}, X_{0}$ and $Y_{0}$ such that

$$
\kappa\left(X_{0}\right) \widehat{I}^{\eta}\left(X_{0}, f\right)=c \widehat{I}\left(Y_{0}, f^{\prime}\right) .
$$

In the following, we choose some global data.

Fields We choose a number field $k$, a quadratic field extension $k^{\prime}$ of $k$, and a quaternion algebra $\mathbb{D}$ over $k$ containing $k^{\prime}$ so that:

1. $k$ is totally imaginary;

2. there exists a finite place $w$ of $k$ such that $k_{w} \simeq F, k_{w}^{\prime} \simeq E$ and $\mathbb{D}\left(k_{w}\right) \simeq \mathrm{D}$;

3. there exists another finite place $u$ of $k$ such that $u$ is inert in $k^{\prime}$.

Such a number field $k$ and a quaternion algebra $\mathbb{D}$ do exist (cf. [Wa3, Proposition in $\S 11.1])$. From now on, we identify $k_{w}$ with $F, k_{w}^{\prime}$ with $E$ and $\mathbb{D}\left(k_{w}\right)$ with $\mathrm{D}$. Denote by $\mathbb{A}$ the ring of adeles of $k$, by $\mathcal{O}_{k}$ the ring of integers of $k$, and $\mathcal{O}_{k^{\prime}}$ the ring of integers of $k^{\prime}$. Fix a continuous character $\mathbb{A} / k$ whose local component at $w$ is our fixed character $\psi$ of $k_{w}$. Denote by $\psi$ this global character, when there is no confusion.

Groups We define the global symmetric pairs $(\mathbf{G}, \mathbf{H})$ and $\left(\mathbf{G}^{\prime}, \mathbf{H}^{\prime}\right)$ over $k$ with respect to $k^{\prime}$ and $\mathbb{D}$ similarly as the local case. We still use $\mathfrak{h}$ and $\mathfrak{h}^{\prime}$ to denote the Lie algebras of $\mathbf{H}$ and $\mathbf{H}^{\prime}$ respectively, use $\mathfrak{s}$ and $\mathfrak{s}^{\prime}$ to denote the global Lie algebras corresponding to $(\mathbf{G}, \mathbf{H})$ and $\left(\mathbf{G}^{\prime}, \mathbf{H}^{\prime}\right)$ respectively, if there is no confusion. Thus $X_{0} \in \mathfrak{s}_{\mathrm{rs}}\left(k_{w}\right)$ and $Y_{0} \in \mathfrak{s}_{\mathrm{rs}}^{\prime}\left(k_{w}\right)$.

Places Denote by $V$ (resp. $V_{\infty}, V_{\mathrm{f}}$ ) the set of all (resp. archimedean, nonarchimedean) places of $k$. Fix two $\mathcal{O}_{k}$-lattices: $\mathbf{L}=\mathfrak{g l}_{n}\left(\mathcal{O}_{k}\right) \oplus \mathfrak{g l}_{n}\left(\mathcal{O}_{k}\right) \subset \mathfrak{s}(k)$ and $\mathbf{L}^{\prime}=\mathfrak{g l}_{n}\left(\mathcal{O}_{k^{\prime}}\right) \subset \mathfrak{s}^{\prime}(k)$. For each $v \in V_{\mathrm{f}}$, put $\mathbf{L}_{v}=\mathbf{L} \otimes_{\mathcal{O}_{k}} \mathcal{O}_{k, v}, \mathbf{L}_{v}^{\prime}=$ $\mathbf{L}^{\prime} \otimes \mathcal{O}_{k} \mathcal{O}_{k, v}$. We fix a finite set $S \subset V$ such that:

1. $S$ contains $u, w$ and $V_{\infty}$;

2. for each $v \in V-S$, everything is unramified, i.e. $\mathbf{G}$ and $\mathbf{G}^{\prime}$ are unramified over $k_{v}, \mathbf{L}_{v}$ and $\mathbf{L}_{v}^{\prime}$ are self-dual with respect to $\psi_{v}$ and $\langle$,$\rangle .$

We denote by $S^{\prime}$ the subset $S-V_{\infty}-\{w\}$ of $S$.

Orbits For each $v \in V_{\mathrm{f}}$, we choose an open compact subset $\Omega_{v} \subset \mathfrak{s}^{\prime}\left(k_{v}\right)$ such that:

1. if $v=w$, we require that: $Y_{0} \in \Omega_{w} \subset \mathfrak{s}_{\mathrm{rs}}^{\prime}\left(k_{w}\right), \widehat{I}\left(\cdot, f^{\prime}\right)$ is constant on $\Omega_{w}$, and $\kappa(\cdot) \widehat{I}^{\eta}(\cdot, f)$ is constant and hence equal to $\kappa\left(X_{0}\right) \widehat{I}^{\eta}\left(X_{0}, f\right)$ on the set of $X \in \mathfrak{s}_{\mathrm{rs}}\left(k_{w}\right)$ which matches an element $Y$ in $\Omega_{w}$, 
2. if $v=u$, we require $\Omega_{u} \subset \mathfrak{s}_{\mathrm{ell}}^{\prime}\left(k_{u}\right)$;

3. if $v \in S$ but $v \neq w, u$, choose $\Omega_{v}$ to be any open compact subset;

4. if $v \in V_{\mathrm{f}}-S$, let $\Omega_{v}=\mathbf{L}_{v}^{\prime}$.

Then by the strong approximation theorem, there exists $Y^{0} \in \mathfrak{s}^{\prime}(k)$ such that $Y^{0} \in \Omega_{v}$ for each $v \in V_{\mathrm{f}}$. Furthermore, by the condition (ii) above, $Y^{0} \in \mathfrak{s}_{\text {ell }}^{\prime}(k)$. Take an element $X^{0} \in \mathfrak{s}_{\text {ell }}(k)$ such that $X^{0} \leftrightarrow Y^{0}$.

Functions For each $v \in V$, we choose functions $\phi_{v} \in \mathcal{S}\left(\mathfrak{s}\left(k_{v}\right)\right)$ and $\phi_{v}^{\prime} \in$ $\mathcal{S}\left(\mathfrak{s}^{\prime}\left(k_{v}\right)\right)$ as follows:

1. if $v=w$, let $\phi_{v}=f$ and $\phi_{v}^{\prime}=f^{\prime}$;

2. if $v \in S^{\prime}$, by Proposition 7.6, we require that:

- if $X_{v} \in \operatorname{Supp}\left(\phi_{v}\right)$, there exists $Y_{v} \in \mathfrak{c}_{Y^{0}}^{\prime}\left(k_{v}\right)$ such that $X_{v} \leftrightarrow Y_{v}$, where we denote by $\mathfrak{c}_{Y^{0}}^{\prime}$ the Cartan subspace in $\mathfrak{s}^{\prime}$ containing $Y^{0}$;

- if $Y_{v} \in \operatorname{Supp}\left(\phi_{v}^{\prime}\right)$, there exists $Y_{v}^{\prime} \in \mathfrak{c}_{Y^{0}}^{\prime}\left(k_{v}\right)$ such that $Y_{v}$ and $Y_{v}^{\prime}$ are $\mathbf{H}^{\prime}\left(k_{v}\right)$-conjugate;

- $\phi_{v}$ is a transfer of $\phi_{v}^{\prime}$;

- $\kappa_{v}\left(X^{0}\right) \widehat{I}^{\eta}\left(X^{0}, \phi_{v}\right)=c_{v} \widehat{I}\left(Y^{0}, \phi_{v}^{\prime}\right) \neq 0$, where $c_{v}=\gamma_{\psi}\left(\mathfrak{h}\left(k_{v}\right)\right) \gamma_{\psi}\left(\mathfrak{h}^{\prime}\left(k_{v}\right)\right)^{-1}$;

3. for $v \in V-S$, set $\phi_{v}=\mathbf{1}_{\mathbf{L}_{v}}, \phi_{v}^{\prime}=\mathbf{1}_{\mathbf{L}_{v}^{\prime}}$; then $\phi_{v}=\widehat{\phi}_{v}, \phi_{v}^{\prime}=\widehat{\phi_{v}^{\prime}}$, and by Lemma 5.18 we have

$$
\kappa_{v}\left(X^{0}\right) \widehat{I}^{\eta}\left(X^{0}, \phi_{v}\right)=\kappa_{v}\left(X^{0}\right) I^{\eta}\left(X^{0}, \phi_{v}\right)=I\left(Y^{0}, \phi_{v}^{\prime}\right)=\widehat{I}\left(Y^{0}, \phi_{v}^{\prime}\right) ;
$$

4. for $v \in V_{\infty}$, identifying $\left(\mathbf{H}\left(k_{v}\right), \mathfrak{s}\left(k_{v}\right)\right)$ with $\left(\mathbf{H}^{\prime}\left(k_{v}\right), \mathfrak{s}^{\prime}\left(k_{v}\right)\right)$, we choose $\phi_{v}=\phi_{v}^{\prime} \in \mathcal{S}\left(\mathfrak{s}\left(k_{v}\right)\right)$ such that:

- $\widehat{I}^{\eta}\left(X^{0}, \phi_{v}\right)=\widehat{I}\left(Y^{0}, \phi_{v}^{\prime}\right) \neq 0$;

- if $X \in \mathfrak{s}(k)$ is $\mathbf{H}\left(k_{v}\right)$-conjugate to an element in the support of $\widehat{\phi_{v}}$ at each place $v \in V$, then $X$ is $\mathbf{H}(k)$-conjugate to $X^{0}$;

- if $Y \in \mathfrak{s}^{\prime}(k)$ is $\mathbf{H}^{\prime}\left(k_{v}\right)$-conjugate to an element in the support of $\widehat{\phi_{v}^{\prime}}$ at each place $v \in V$, then $Y$ is $\mathbf{H}^{\prime}(k)$-conjugate to $Y^{0}$.

This is possible. The key point is that, by invariant theory, we have natural maps (cf. Remark 5.3)

$$
\mathfrak{s}^{\prime} / \mathbf{H}^{\prime} \hookrightarrow \mathfrak{s} / \mathbf{H} \longrightarrow \mathbf{A}_{k}^{n},
$$

where $\mathbf{A}_{k}^{n}$ is the $n$-dimensional affine space over $k$ so that $\mathbf{A}_{k}^{n}=\operatorname{Spec}\left(\mathcal{O}(\mathfrak{s})^{\mathbf{H}}\right)$. We refer the reader to [Wa3, Lemme in $\S 10.7]$ for the proof in the endoscopic case, and a similar argument is also valid here.

Set $\phi \in \mathcal{S}(\mathfrak{s}(\mathbb{A}))$ and $\phi^{\prime} \in \mathcal{S}\left(\mathfrak{s}^{\prime}(\mathbb{A})\right)$ to be:

$$
\phi=\prod_{v \in V} \phi_{v}, \quad \phi^{\prime}=\prod_{v \in V} \phi_{v}^{\prime} .
$$


Final proof According to the conditions on $\phi_{u}$ (resp. $\phi_{u}^{\prime}$ ), we know that if $X \in \mathfrak{s}(k)$ (resp. $Y \in \mathfrak{s}^{\prime}(k)$ ) is such that $X \in \operatorname{Supp}(\phi)^{\mathbf{H}(\mathbb{A})}$ (resp. $Y \in$ $\left.\operatorname{Supp}\left(\phi^{\prime}\right)^{\mathbf{H}^{\prime}(\mathbb{A})}\right)$, then $X \in \mathfrak{s}_{\text {ell }}(k)\left(\right.$ resp. $\left.Y \in \mathfrak{s}_{\text {ell }}^{\prime}(k)\right)$. Here we use $\operatorname{Supp}(\phi)^{\mathbf{H}(\mathbb{A})}$ to denote the union of $\mathbf{H}(\mathbb{A})$-orbits intersecting $\operatorname{Supp}(\phi)$, and $\operatorname{Supp}\left(\phi^{\prime}\right)^{\mathbf{H}^{\prime}(\mathbb{A})}$ to denote the union of $\mathbf{H}^{\prime}(\mathbb{A})$-orbits intersecting $\operatorname{Supp}\left(\phi^{\prime}\right)$. Suppose that $X \in$ $\mathfrak{s}_{\text {ell }}(k)$ is such that

$$
I^{\eta}(X, \phi)=\prod_{v \in V} I^{\eta}\left(X, \phi_{v}\right) \neq 0 .
$$

Then, by the conditions on $\phi_{v}, X$ comes from $\mathfrak{s}^{\prime}\left(k_{v}\right)$ at each place $v$ not equal to $w$. We claim that $X$ must come from $\mathfrak{s}^{\prime}(k)$. If not, there exists at least two places $v_{1}$ and $v_{2}$ such that $X$ does not come from $\mathfrak{s}^{\prime}\left(k_{v}\right)$, which is a contradiction. Therefore we have

$$
I^{\eta}(\phi)=I\left(\phi^{\prime}\right),
$$

since $\phi_{v}$ is a transfer of $\phi_{v}^{\prime}$ at each place $v$ not equal to $w$ and is a partial transfer of $\phi_{v}^{\prime}$ at the place $v=w$ by the requirements we have imposed.

On the other hand, according to the conditions on $\widehat{\phi_{v}}$ and $\widehat{\phi_{v}^{\prime}}$, we know that if $X \in \mathfrak{s}(k)\left(\right.$ resp. $\left.Y \in \mathfrak{s}^{\prime}(k)\right)$ is such that $X \in \operatorname{Supp}(\widehat{\phi})^{\mathbf{H}(\mathbb{A})}$ (resp. $Y \in$ $\left.\operatorname{Supp}\left(\widehat{\phi}^{\prime}\right)^{\mathbf{H}^{\prime}(\mathbb{A})}\right)$ then $X$ is $\mathbf{H}(k)$-conjugate to $X^{0}$ (resp. $Y$ is $\mathbf{H}^{\prime}(k)$-conjugate to $\left.Y^{0}\right)$.

By Poisson summation formula, we have

$$
\sum_{X \in \mathfrak{s}(k)} \phi\left(X^{h}\right)=\sum_{X \in \mathfrak{s}(k)} \widehat{\phi}\left(X^{h}\right), \quad \forall h \in \mathbf{H}(\mathbb{A}),
$$

and

$$
\sum_{Y \in \mathfrak{s}^{\prime}(k)} \phi^{\prime}\left(Y^{h}\right)=\sum_{Y \in \mathfrak{s}^{\prime}(k)} \widehat{\phi}^{\prime}\left(Y^{h}\right), \quad \forall h \in \mathbf{H}^{\prime}(\mathbb{A}) .
$$

Therefore, by the conditions on $\phi$ and $\phi^{\prime}$, we have

$$
I^{\eta}(\phi)=I^{\eta}(\widehat{\phi}), \quad I\left(\phi^{\prime}\right)=I\left(\widehat{\phi}^{\prime}\right) .
$$

Hence we obtain

$$
I^{\eta}(\widehat{\phi})=I\left(\widehat{\phi}^{\prime}\right)
$$

or equivalently,

$$
\tau\left(\mathbf{H}_{X^{0}}\right) \prod_{v \in V} \kappa_{v}\left(X^{0}\right) \widehat{I}^{\eta}\left(X^{0}, \phi_{v}\right)=\tau\left(\mathbf{H}_{Y^{0}}^{\prime}\right) \prod_{v \in V} \widehat{I}\left(Y^{0}, \phi_{v}^{\prime}\right) .
$$

Note that for $v \in V-S$, we have

$$
\kappa_{v}\left(X^{0}\right) \widehat{I}^{\eta}\left(X^{0}, \phi_{v}\right)=\widehat{I}\left(Y^{0}, \phi_{v}^{\prime}\right) \neq 0,
$$

and for almost all $v \in V-S$,

$$
\kappa_{v}\left(X^{0}\right) \widehat{I}^{\eta}\left(X^{0}, \phi_{v}\right)=\widehat{I}\left(Y^{0}, \phi_{v}^{\prime}\right)=1 .
$$

For $v \in S^{\prime}$ and $v \in V_{\infty}$, we have

$$
\kappa_{v}\left(X^{0}\right) \widehat{I}^{\eta}\left(X^{0}, \phi_{v}\right)=c_{v} \widehat{I}\left(Y^{0}, \phi_{v}^{\prime}\right) \neq 0 .
$$


Therefore

$$
\kappa_{w}\left(X^{0}\right) \widehat{I}^{\eta}\left(X^{0}, f\right)=c \widehat{I}\left(Y^{0}, f^{\prime}\right),
$$

where

$$
c=\left(\prod_{v \in S^{\prime}} c_{v}\right)^{-1}=\prod_{v \in S^{\prime}} \gamma_{\psi}\left(\mathfrak{h}\left(k_{v}\right)\right)^{-1} \gamma_{\psi}\left(\mathfrak{h}^{\prime}\left(k_{v}\right)\right) .
$$

Notice that if $v \in V_{\infty}$ or $v \in V-S$,

$$
\gamma_{\psi}\left(\mathfrak{h}\left(k_{v}\right)\right)=\gamma_{\psi}\left(\mathfrak{h}^{\prime}\left(k_{v}\right)\right)=1 .
$$

Also notice that

$$
\prod_{v \in V} \gamma\left(\mathfrak{h}\left(k_{v}\right)\right)=\prod_{v \in V} \gamma_{\psi}\left(\mathfrak{h}^{\prime}\left(k_{v}\right)\right)=1
$$

Therefore

$$
c=\gamma_{\psi}\left(\mathfrak{h}\left(k_{w}\right)\right) \gamma_{\psi}\left(\mathfrak{h}^{\prime}\left(k_{w}\right)\right)^{-1}
$$

Since

$$
\kappa_{w}\left(X_{0}\right) \widehat{I}^{\eta}\left(X_{0}, f\right)=\kappa_{w}\left(X^{0}\right) \widehat{I}^{\eta}\left(X^{0}, f\right), \quad \widehat{I}\left(Y_{0}, f^{\prime}\right)=\widehat{I}\left(Y^{0}, f^{\prime}\right),
$$

we complete the proof of the theorem.

Acknowledgements This work was supported by the National Key Basic Research Program of China (No. 2013CB834202). Needless to say, the remarkable work of Jean-Loup Waldspurger on endoscopic transfer has a huge influence on this article. The author is very grateful to Wei Zhang for suggesting this problem and sharing the idea that Waldspurger's method might apply to this situation, to Binyong Sun for proving Theorem 8.4 which is crucial for our method. He also thanks Kimball Martin for communicating their work [FMW] and sending their preprint, and thanks Wen-Wei Li for helpful discussions. He expresses gratitude to Ye Tian and Linsheng Yin for their constant encouragement and support. He would like to thank the anonymous referee for explaining how to prove Theorem 8.1, which improves the main result of the article greatly, and many other useful comments.

\section{References}

[AG] A. Aizenbud and D. Gourevitch, Generalized Harish-Chandra descent, Gelfand pairs, and an Archimedean analog of Jacquet-Rallis's theorem, with an appendix by the authors and Eitan Sayag, Duke Math. J. 149 (2009), no. 3, 509-567.

[AC] J. Arthur and L. Clozel, Simple algebras, base change, and advanced theory of the trace formula, Ann. Math. Study 120, Princeton Univ. Press, 1989.

[FMW] B. Feigon, K. Martin and D. Whitehouse, Periods and nonvanishing of central L-values for GL(2n), arXiv:1308.2253.

[FM] M. Furusawa and K. Martin, Local root numbers, Bessel models, and a conjecture of Guo and Jacquet, Journal of Number Theory, Special Issue in honor of Steve Rallis. 
[FJ] S. Friedberg and H. Jacquet, Linear periods, J. Reine Angew. Math. 443 (1993), 91-139.

[GGP] W. T. Gan, B. H. Gross and D. Prasad, Symplectic local root numbers, central critical L-values, and restriction problems in the representation theory of classical groups, Astérisque 346 (2012), 1-109.

[Gu1] J. Guo, On a generalization of a result of Waldspurger, Canad. J. Math. 48 (1996), 105-142.

[Gu2] J. Guo, Uniqueness of generalized Waldspurger model for GL(2n), Pacific J. Math. 180 (1997), no. 2, 273-289.

[HC1] Harish-Chandra, (notes by G. van Dijk) Harmonic analysis on reductive p-adic groups, Lecture Notes in Mathematics, vol. 162, Springer, 1970.

[HC2] Harish-Chandra, Admissible invariant distributions on reductive p-adic groups. Notes by S. DeBaker and P. J. Sally, University Lecture Series, vol. 16, AMS, 1999.

[Ha] Neal Harris, A refined Gross-Prasad conjecture for unitary groups, PhD Thesis, 2011, UCSD.

[II] A. Ichino and T. Ikeda, On the periods of automorphic forms on special orthogonal groups and the Gross-Prasad conjecture, Geom. Funct. Anal. 19 (2010), no. 5, 1378-1425.

[Ja1] H. Jacquet, Sur un résultat de Waldspurger, Ann. Sci. École Norm. Sup. 19 (1986), 185-229.

[Ja2] H. Jacquet, Smooth transfer of Kloosterman integrals, Duke Math. J. 120 (2003), no. 1, 121-152.

[JR] H. Jacquet and S. Rallis, Uniqueness of linear periods, Compositio Math. 102 (1996), 65-123.

[Ko] R. Kottwitz, Harmonic analysis on reductive p-adic grups and Lie algebras, in Harmonic analysis, the trace formula, and Shimura varieties, 393-522, Clay Math. Proc., 4, Amer. Math. Soc., Providence, RI, 2005.

[RR] C. Rader and S. Rallis, Spherical characters on p-adic symmetric spaces, Amer. J. Math. 118 (1996), no. 1, 91-178.

[SV] Y. Sakellaridis and A. Venkatesh, Periods of harmonic analysis on spherical varieties, arXiv:1203.0039.

[Wa1] J.-L. Waldspurger, Sur les valeurs de certaines fonctions L automorphes en leur centre de symétrie, Compositio Math. 54 (1985), 173-242.

[Wa2] J.-L. Waldspurger, Une formule des traces locale pour les algèbres de Lie p-adiques, J. Reine Angew. Math. 465 (1995), 41-99.

[Wa3] J.-L. Waldspurger, Le lemme fondamental implique le transfert, Compositio Math. 105 (1997), 153-236. 
[Zhc] C. Zhang, On linear periods, Math. Z., to appear.

[Zhw1] W. Zhang, Fourier transform and the global Gan-Gross-Prasad conjecture for unitary groups, Ann. Math. 180 (2014), no. 3, 971-1049.

[Zhw2] W. Zhang, Automorphic period and the central value of Rankin-Selberg L-function, J. Amer. Math. Soc. 27 (2014), 541-612.

Chong Zhang

School of Mathematical Sciences, Beijing Normal University,

Beijing 100875, P. R. China.

E-mail address: zhangchong@bnu.edu.cn 